\title{
Anisotropy in CdSe Quantum Rods
}

\author{
by \\ Liang-shi Li \\ B. S. (University of Science \& Technology of China, China) 1994
}

A dissertation submitted in partial satisfaction of the

requirements for the degree of

\author{
Doctor of Philosophy \\ in \\ Chemistry \\ in the \\ GRADUATE DIVISION \\ of the \\ UNIVERSITY OF CALIFORNIA, BERKELEY \\ Committee in Charge \\ Professor A. Paul Alivisatos, Chair \\ Professor Norman E. Phillips \\ Professor Dung-Hai Lee
}

Spring 2003 
Anisotropy in CdSe Quantum Rods

@ 2003

by

Liang-shi Li 


\author{
Abstract \\ Anisotropy in CdSe Quantum Rods \\ by \\ Liang-shi Li \\ Doctor of Philosophy in Chemistry \\ University of California, Berkeley \\ Professor A. Paul Alivisatos, Chair
}

The size-dependent optical and electronic properties of semiconductor nanocrystals have drawn much attention in the past decade, and have been very well understood for spherical ones. The advent of the synthetic methods to make rod-like CdSe nanocrystals with wurtzite structure has offered us a new opportunity to study their properties as functions of their shape. This dissertation includes three main parts: synthesis of CdSe nanorods with tightly controlled widths and lengths, their optical and dielectric properties, and their large-scale assembly, all of which are either directly or indirectly caused by the uniaxial crystallographic structure of wurtzite CdSe.

The hexagonal wurtzite structure is believed to be the primary reason for the growth of CdSe nanorods. It represents itself in the kinetic stabilization of the rod-like particles over the spherical ones in the presence of phosphonic acids. By varying the composition of the surfactant mixture used for synthesis we have achieved tight control of the widths and lengths of the nanorods.

The synthesis of monodisperse CdSe nanorods enables us to systematically study their size-dependent properties. For example, room temperature single particle fluorescence spectroscopy has shown that nanorods emit linearly polarized 
photoluminescence. Theoretical calculations have shown that it is due to the crossing between the two highest occupied electronic levels with increasing aspect ratio. We also measured the permanent electric dipole moment of the nanorods with transient electric birefringence technique. Experimental results on nanorods with different sizes show that the dipole moment is linear to the particle volume, indicating that it originates from the non-centrosymmetric hexagonal lattice.

The elongation of the nanocrystals also results in the anisotropic inter-particle interaction. One of the consequences is the formation of liquid crystalline phases when the nanorods are dispersed in solvent to a high enough concentration. The preparation of the stable liquid crystalline solution of CdSe nanorods is described, as well as the large-scale alignment of the nanorods by taking advantage of the longrange orientational correlation in the liquid crystals. In addition, we investigated the phase diagram of the nanorod solution, as a step toward understanding the possible role of the long-range attractive interaction between the nanorods in the formation of lyotropic liquid crystals. 
To My Parents and $\mathrm{Na}$ 


\section{Acknowledgement}

It is a great relief to see myself going through the graduate student life in Berkeley. The past six years have been full of frustrations, struggles and excitements. I feel very fortunate to have Prof. A. Paul Alivisatos as my advisor. His patience and trust have been very important for me to develop my own thoughts, and his attitude towards science and his open-mindedness have greatly influenced my perception about scientific research.

I would like to acknowledge all the members of the Alivisatos group. I very much enjoyed working with them, exchanging ideas and learning from each other. My thanks especially go to Deborah Aruguete for taking shift at the Advanced Light Source with me, Benjamin Boussert for proofreading almost all the papers I wrote, Shara Williams for helping me with English, Dr. Victor F. Puntes, Dr. Daniele Gerion, Dr. Jennifer Cha, Dr. Maria Casula and others for sharing the laughs and optimisms, and I wish them good luck in their careers. I also greatly appreciate the assistance from the past group members, especially Dr. Xiaogang Peng for his advice on my synthetic work in the past years, Dr. Liberato Manna for helping me get started on the synthesis of nanorods, Dr. Weidong Yang and Dr. Jiangtao Hu for the collaboration in the photoluminescence measurements and teaching me software and electronics. Thanks also to our administrative assistants, Rita Tidwell and Helen Cameron, for making the purchases faster and my life easier.

The work described in this thesis would not have been possible without the help of the people in the glass, electronics and machine shops of the College of Chemistry. 
Especially, Tom Lawhead has been very helpful in designing and making the glass gaskets that are extremely important in the liquid crystal project.

I had the honor to work with some wonderful collaborators outside our group. I would like to thank Dr. Malgorzata Marjanska for teaching me NMR and working with me on the NMR measurements. Thanks also to Mario Yosa and Dr. Youli Li at the Materials Research Lab of UC Santa Barbara for their assistance in small angle x-ray scattering measurements, to Paul Books at the analytical facility of the College of Natural Resources for his assistance in the elemental analysis, and to Dr. Matthew Marcus at beamline 10.3.2. of the Advanced Light Source for the work and discussion on the polarized EXAFS experiments.

I would like to thank Mary and George Breunig for their hospitality. They had helped me a great deal settle down in this country and made their house much like a home to me. I also thank the late Ms. Billy Tobey for sharing her wisdom and experience. Her spirit despite her age had made me realize that it is never too late to learn anything.

I would like to thank my friend Richard Wong for the time we spent together these years, arguing about politics and history from different points of view, which made my life in Berkeley much more enjoyable.

I am very grateful to my parents, my brother and sister for their support. I thank my love, $\mathrm{Na} \mathrm{Ji}$ for her encouragement and help, both in research and in daily life. I am so lucky to have her in my life. I wouldn't have gone this far without them. 


\section{Table of Contents}

CHAPTER1. INTRODUCTION.................................................

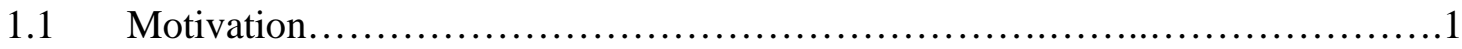

1.2 Quantum confinement...................................................

1.3 Effective mass approximation model.................................... 3

1.4 Outline of the thesis....................................................

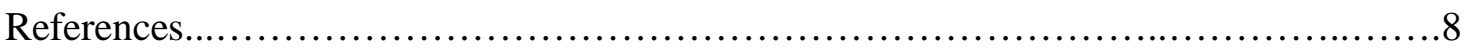

CHAPTER 2. CONTROLLED SYNTHESIS OF CdSe NANORODS....................11

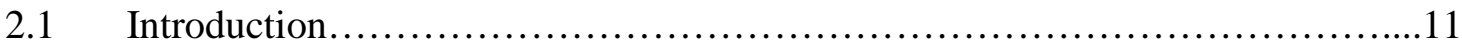

2.2 Proposed growth mechanisms of CdSe nanorods.........................13

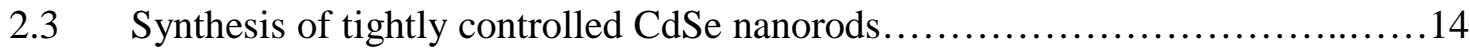

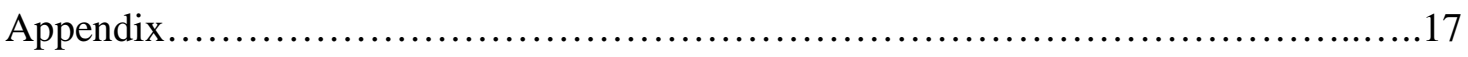

References.....................................................................

CHAPTER 3. OPTICAL PROPERTIES OF CdSe NANORODS........................20

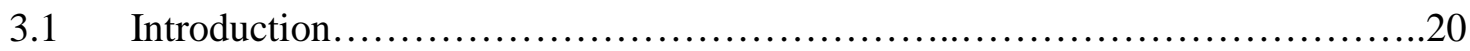

3.2 Size dependence of photoluminescence spectra of CdSe nanorods..............21

3.3 Dependence of the global Stokes shift on the aspect ratio of CdSe nanorods..... 23

3.4 Linear polarization of the photoluminescence of CdSe nanorods................24

3.5 Evolution of the electronic structure of CdSe nanorods with aspect ratio-

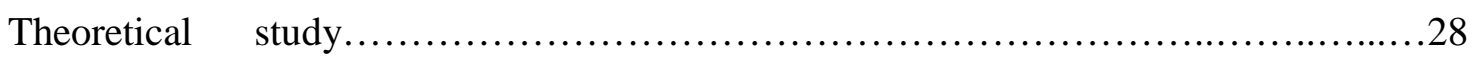

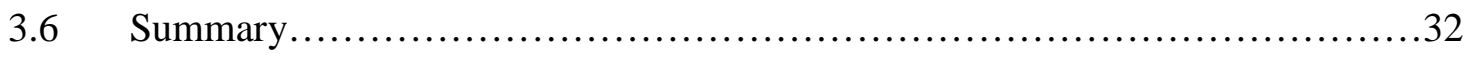

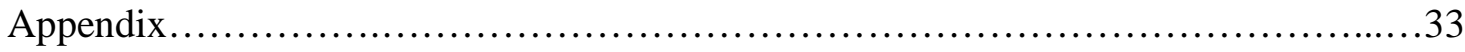

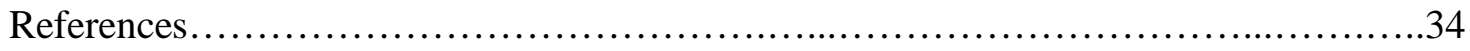


CHAPTER 4. PERMANENT ELECTRIC DIPOLE MOMENT OF CdSe

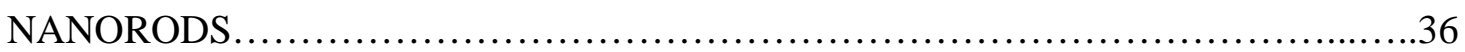

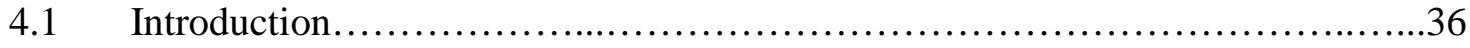

4.2 Electric Birefringence Measurement...................................... 38

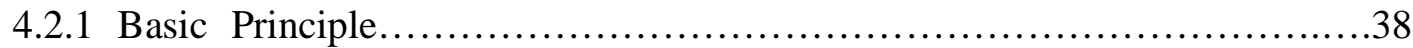

4.2.2 Experimental setup...................................................40

4.2.2.1 Calibration of the setup...............................................

4.3 Permanent Dipole Moment of CdSe Nanorods..............................42

Appendix .............................................................. 49

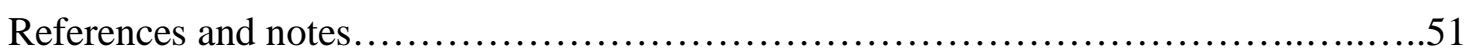

CHAPTER 5. ASSEMBLY OF NANORODS ON A SUBSTRATE....................53

$5.1 \quad$ Motivation............................................................... 53

5.2 Alignment of CdSe nanorods on an aligned substrate........................55

5.3 Superlattices of CdSe nanorods by slow solvent evaporation...................57

References.....................................................................

CHAPTER 6. LYOTROPIC LIQUID CRYSTALLINE PHASE OF CdSe NANOROD

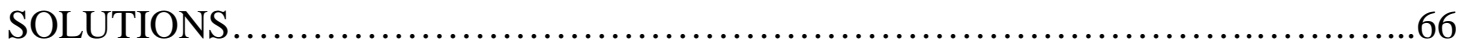

$6.1 \quad$ Motivation.............................................................66

6.2 Liquid crystal.....................................................67

6.2.1 Line defects in liquid crystals-Disclinations.........................67

6.2.2 Lyotropic liquid crystalline phases......................................69

6.2.2.1 Onsager's Theory-Hard rod model.................................70

6.2.2.2 Flory's theories............................................... 71

6.3 Spontaneous optical anisotropy in CdSe nanorod solution.....................74

6.4 Stable liquid crystalline solution of CdSe nanorods..........................77

6.4.1 Thermal-reversible gelation in concentrated solution of CdSe nanorods.........78 
6.4.1.1 Phase separation mechanisms and gelation...........................78

6.4.1.2 Gelation in concentrated CdSe solution...............................81

6.4.2 Stable liquid crystalline solution of CdSe nanorods..........................81

6.5 Alignment of CdSe nanorods............................................. 86

6.5.1 Surface induced alignment.......................................... 86

6.5.2 Alignment of CdSe nanorods with magnetic field..........................87

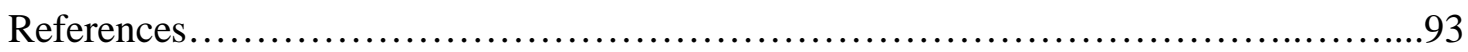

CHAPTER 7. PHASE DIAGRAM OF SOLUTION OF CdSe NANORODS............96

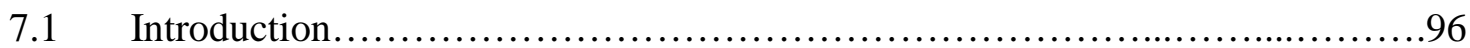

7.2 Isotropic-liquid crystalline phase diagram of the solution of CdSe nanorods......98

7.2.1 Determination of the concentration of CdSe nanorods.......................100

7.2.2 Temperature dependence of the phase diagram...........................101

7.2.3 Discussion-Comparison with rigid model.................................103

7.3 Summaries and future work................................................ 104

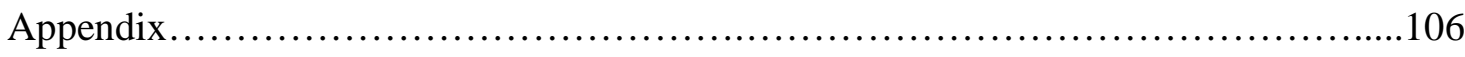

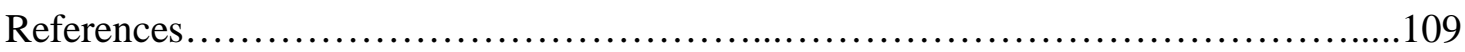

CHAPTER 8. CONCLUSIONS................................................... 111 


\section{Chapter1 Introduction}

\subsection{Motivation}

Semiconductor nanocrystals ${ }^{1,2}$, also referred as "artificial atoms", have been a subject of intensive studies due to their size-dependent electronic and optical properties, and their potential to be used as active components in electro-optical devices. The colloidal nanocrystals synthesized by wet chemistry methods ${ }^{3,4}$ have in particular drawn a lot of attention because of their chemical processibility and their capability to be incorporated into various matrices such as conducting polymers ${ }^{5,6,7,8}$ and biological systems ${ }^{9,10,11,12}$. In the past decades, the studies of nearly spherical CdSe nanocrystals with the wurtzite lattice structure have played an important role in our understanding of quantum confinement $^{13,14}$ and of the growth mechanism of nanocrystals ${ }^{15,16}$. The recent development in the synthetic methods have made nanoparticles with nonspherical shapes possible ${ }^{17,18,19,20,21,22}$, and the breaking of the spherical symmetry should be accompanied by anisotropic properties. This thesis covers some systematic studies on rodlike CdSe nanocrystals. We believe that the hexagonal crystallographic structure is the primary origin of the anisotropy in these rodlike nanocrystals, including the anisotropy of their shape, properties, and assembly, as described in the following chapters.

\subsection{Quantum Confinement}


It has been well known that when the size of the semiconductor crystals is comparable to the bulk exciton Bohr radius (5.6 $\mathrm{nm}$ for $\mathrm{CdSe}$ ), the confinement of the charge carriers alters the electronic band structure of the crystals. Due to the lifting of the translational symmetry, the momentum $\boldsymbol{k}$ of the electrons is not a good quantum number any more, and the originally continuous energy levels become discrete. Together with the mixing of the states with different $\boldsymbol{k}$ values, the band gap increases. As a result there is a blue shift in both the electronic absorption spectra and the photoluminescence spectra.

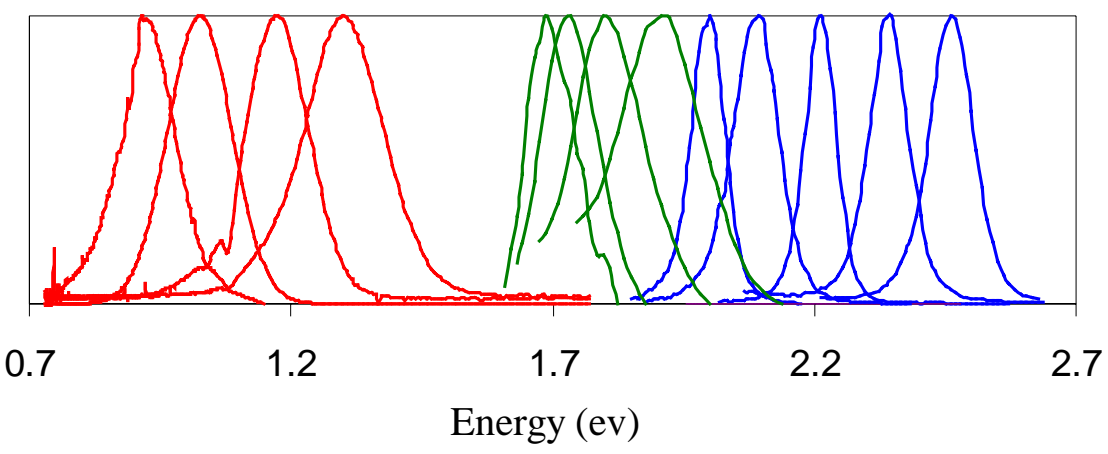

Fig. 1.1 The photoluminescence spectra of InAs (red), InP (green) and CdSe (blue) spherical nanocrystals with different $\operatorname{sizes}^{9}$. The diameters of the nanocrystals are, from left to right, 6.0, 4.6, 3.6, $2.8,4.6,4.0,3.5,3.0,4.6,3.6,3.1,2.4,2.1 \mathrm{~nm}$.

Figure 1.1 shows the fluorescence spectra of spherical nanocrystals of InAs, InP and CdSe, for each of which the emission can be tuned in different frequency ranges by varying the size of the nanocrystals. Especially for CdSe spherical nanocrystals with diameter ranging from $2.0 \mathrm{~nm}$ to $6.0 \mathrm{~nm}$, the fluorescence spectra cover the whole range of visible light. 


\subsection{Effective Mass Approximation Model}

Besides the blue shift in the absorption and fluorescence spectra of the nanocrystals, quantum confinement also has other consequences, such as changes in the selection rules of electronic transitions and fluorescence lifetime. The theoretical studies of these effects in nearly spherical semiconductor nanocrystals, e.g. the effective mass approximation model, the pseudopotential method ${ }^{23}$ and the tight-binding model ${ }^{24}$, have been very successful in quantitatively explaining the observed experimental results. Here only the effective mass approximation model is introduced, because it has a simple physical picture and will be used to understand the electronic properties of the CdSe nanorods.

The top of the valence band and the bottom of the conduction band of bulk semiconductors with zinc-blende-type and wurtzite-type structures are shown in figure 1.2 respectively. The conduction band consists of $s$ atomic orbitals and the valence band of $p$ atomic orbitals. Due to the spin-orbital coupling, the valence band is split into three subbands: heavy-hole (HH), light-hole ( $\mathrm{LH})$ and split-off (SO) bands. In zinc-blende-type semiconductors, the $\mathrm{HH}$ and $\mathrm{LH}$ bands are degenerate at $\boldsymbol{k}=0$; while in wurtzite-type ones this degeneracy is lifted by the crystal field, and the energy difference at $\boldsymbol{k}=0$ is called the crystal field splitting $\left(\Delta_{c f}\right)$. The three hole subbands are the superpositions of the Bloch functions of $p_{x, y, z}$ orbitals., More specifically, $\mathrm{HH}$ is made of $p_{x, y}$, while LH and SO of both $p_{x, y}$ and $p_{z}$ orbitals $^{25}$. HH, LH and SO subbands have total unit-cell angular momentum of $3 / 2,3 / 2$ and $1 / 2$ respectively, as shown in figure 1.2.

The basic idea of the effective mass approximation in treating the nanocrystals is to use the electronic wave functions of the corresponding bulk material as the basis set to 
study the eigenvalue problem of the "band-mixing" due to the boundary conditions imposed by the finite volume. Because the valence subbands are nearly degenerate, they contribute to most of the complexity when mixed by the confinement. Depending on the actual material and one's interest, further simplification can be made. Since CdSe nanocrystals have a large band gap $(>1.72 \mathrm{eV})$, the hole states and the electron states can be treated separately without considering their mixing. Furthermore, as we are mostly interested in their photoluminescence properties, which only involve the lowest excited states, a four-band (including $\mathrm{HH}$ and $\mathrm{LH}$ ) model is sufficient.

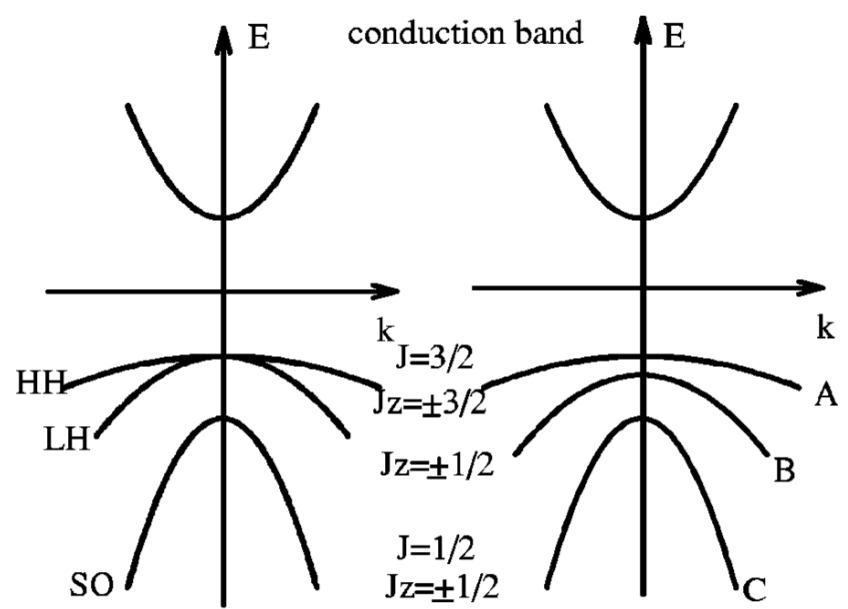

(a) valence band (b)
Fig. 1.2 The schematics of the top of the valence band and the bottom of the conduction band of bulk semiconductors with zinc-blende-type (a) and wurtzitetype (b) structures. The different naming schemes in the literature for the valence sub-bands are shown. The $\mathbf{J}$ and $\mathrm{J}_{z}$ are total angular momentum and its $z$ components after spin-orbital coupling. Note the presence of the crystal field coupling in the wurtzite-type semiconductor.

Within the four-band model, the hole Hamiltonian in hexagonal crystals can be written as $\hat{H}=\hat{H}_{L}+\hat{V}$, where $\hat{H}_{L}$ is the Luttinger Hamiltonian ${ }^{26}$, which can be treated with the spherical approximation, and $\hat{V}$ the anisotropic term caused by the crystal field splitting $\Delta_{c f}(25 \mathrm{meV} \text { for } \mathrm{CdSe})^{27}$. With the spherical nanocrystal considered to be 
surrounded by an infinitely high potential barrier, and when the size quantization of the holes is much larger than $\Delta_{c f}$, the eigenvalue problem for the hole Hamiltonian above can be solved by perturbation methods with the eigenstates of $\hat{H}_{L}$ as the basis set. The basis set now has the total angular momentum $F=L+J$ as a conserved quantity, where $L$ is the angular momentum of the envelope function due to the confinement of the holes in the spherical potential well, and $J$ its total unit-cell angular momentum, as shown in figure $1.2 .^{28}$

The confinement causes the mixing of the $\mathrm{HH}\left(J=3 / 2, J_{\mathrm{z}}= \pm 3 / 2\right)$ and the $\mathrm{LH}(J=$ $3 / 2, J_{z}= \pm 1 / 2$ ) states. It has been shown that the only hole states involved in the photoluminescence of CdSe nanocrystals are those with $F=3 / 2$ and odd parity ${ }^{27}$, which include four degenerate states with $F_{\mathrm{z}}= \pm 1 / 2$ and $\pm 3 / 2$, respectively. The anisotropic term $\hat{V}$ lifts their four-fold degeneracy, and the energy difference, as shown by the perturbation treatment ${ }^{27}$, is

$$
\delta_{3 / 2}-\delta_{1 / 2}=-v \Delta_{c f}
$$

where $v$ is a positive constant depending on the ratio of the effective mass of $\mathrm{HH}$ and $\mathrm{LH}$ but independent of the size of the nanocrystals. Thus $\left|F_{z}\right|=3 / 2$ states are the lowest excited states, because of the nonzero crystal field splitting in the wurtzite structure. Because the ground electronic states have spherical electron distribution $(L=0, J=1 / 2)$, only the heavy hole component $\left(J=3 / 2, J_{\mathrm{z}}= \pm 3 / 2\right)$ in the lowest excited states $(F=3 / 2$, $F_{\mathrm{z}}= \pm 3 / 2$ ) contributes to the dipole-allowed transition between these two energy levels, so that the fluorescence has to be polarized in $a-b$ crystallographic plane; while the 
emission from the $\left|F_{\mathrm{z}}\right|=1 / 2$ states is linearly polarized along the $c$-axis of the nanocrystals.

Further correction was made to account for the non-spherical shape of the CdSe nanocrystals ${ }^{29}$ which are actually synthesized most of the time. Assume the nanoparticles are slightly prolate with the short axes $b=a(1-\mu / 3)$ and the long axis $c=a(1+2 \mu / 3)$, where $a^{3}=b^{2} c$ and $\mu<<1$, the correction to the energy levels with $F_{z}= \pm 3 / 2$ and $F_{z}= \pm 1 / 2$ is

$$
\Delta \delta_{3 / 2}=-\Delta \delta_{1 / 2}=C \mu>0
$$

with the $F_{z}= \pm 3 / 2$ states raised while the $F_{z}= \pm 1 / 2$ states lowered, where $C$ is a material specific constant which is inversely proportional to the square of the diameter of the nanocrystals. This indicates that the shape ellipticity may compensate the crystal field splitting, and for a fixed shape ellipticity, the order of the energy levels may even change for small enough nanocrystals.

Despite its great simplicity, if the electron exchange energy is considered, the effective mass approximation model can quantitatively explain the experimental results on the nearly spherical CdSe nanocrystals, such as the monotonically decreasing resonance Stokes shift with increasing particle $\operatorname{size}^{28}$ and the decreasing fluorescence lifetime under magnetic field at low temperature $(1.7 \mathrm{~K})^{30}$. 


\subsection{Thesis Outline}

The thesis is arranged in the following way. In chapter 2 I describe the effort to synthesize CdSe nanorods with tightly controlled width and length, which is essential for the systematic study of their properties as functions of the widths and lengths. Then I describe the characterization of some of their physical properties, such as their optical properties in chapter 3 and their dielectric properties in chapter 4. Finally I discuss the assembly properties unique to the nanorods due to their anisotropic geometry. This includes the assembly on a substrate described in chapter 5, their formation of liquid crystalline phase in chapter 6 , and our first attempt to map out the phase diagram of the CdSe nanorod solution in order to study their interactions, which is discussed in chapter 7. 


\section{Reference}

\footnotetext{
${ }^{1}$ Alivisatos, A. P. Science 1996, 271 (5251), 933-937.

${ }^{2}$ Brus, L. Appl. Phys. A 1991, 53 (6), 465-474.

${ }^{3}$ Steigerwald, M. L.; Alivisatos, A. P.; Gibson, J. M.; Harris, T.D.; Kortan, R.; Muller, A.
}

J.; Thayer, A. M.; Duncan, T. M.; Douglass, D. C.; Brus, L. E. J. Am. Chem. Soc. 1988, 110 (10), 3046-3050.

${ }^{4}$ Schmid, G. Chem. Rev. 1992, 92 (8), 1709-1727.

${ }^{5}$ Colvin, V. L.; Schlamp, M. C.; Alivisatos, A. P. Nature 1994, 370 (6488), 354-357.

${ }^{6}$ Dabbousi, B. O.; Bawendi, M. G.; Onotsuka, O.; Rubner, M. F.; Appl. Phys. Lett. 1995, $66(11), 1316-1318$.

${ }^{7}$ Huynh, W.U.; Peng, X.; Alivisatos, A.P.; Adv. Mat. 1999, 11 (11), 923-927.

${ }^{8}$ Tessler, N.; Medvedev, V.; Kazes, M.; Kan, S. H.; Banin, U. Science 2002, 295 (5559), 1506-1509.

${ }^{9}$ Bruchez, M.; Moronne, M.; Gin, P.; Weiss, S.; Alivisatos, A. P. Science 1998, 281 (5358), 2013-2016.

${ }^{10}$ Chan, W. C. W.; Nie, S. M. Science 1998, 281 (5358), 2016-2019.

${ }^{11}$ Loweth, C. J.; Caldwell, W.B.; Peng, X.; Alivisatos, A.P.; Schultz, P.G. Angew. Chem., Int. Ed. Engl. 1999, 38 (12), 1808-1812.

${ }^{12}$ Parak, W. J.; Boudreau, R.; Le Gros, M.; Gerion, D.; Zanchet, D.; Micheel, C. M.;

Williams, S. C.; Alivisatos, A. P. Adv. Mat. 2002, 14 (12), 882-885.

${ }^{13}$ Efros, A. L.; Rosen, M. Ann. Rev. Mat. Sci. 2000, 30, 475-521.

${ }^{14}$ Nirmal, M.; Norris, D. J.; Bawndi, M. G.; Efros, A. L.; Rosen, M. Phy. Rev. Lett. 1995, 75 (20), 3728-3781. 
${ }^{15}$ Murray, C. B., Norris, D. J. and Bawendi, M. G. J. Am. Chem. Soc. 1993, 115 (19), $8706-8715$.

${ }^{16}$ Peng, X. G.; Wickham, J.; Alivisatos, A. P. J. Am. Chem. Soc. 1998, 120 (21), 53435344.

${ }^{17}$ Li, M.; Schnablegger, H.; Mann, S. Nature 1999, 402 (6760), 393-395.

${ }^{18}$ Peng, X.; Manna, L.; Yang, W.; Wickham, J.; Scher, E.; Kadavanich, A.; Alivisatos, A. P. Nature 2000, 404 (6773), 59-61.

${ }^{19}$ Chang, S.; Shih, C.; Chen, C.; Lai, W.; Wang, C. R. C. Langmuir 1999, 15 (3), $701-$ 709.

${ }^{20}$ Puntes, V. F.; Zanchet, D.; Erdonmez, C. K.; Alivisatos, A. P. J. Am. Chem. Soc. 2002, $124(43), 12874-12880$.

${ }^{21}$ Manna, L.; Scher E. C.; Alivisatos, A. P. J. Am. Chem. Soc. 2000, 122 (51), 1270012706.

${ }^{22}$ Jun, Y.; Lee S., Kang, N.; Cheon, J. J. Am. Chem. Soc. 2001, 123 (21), 5150-5151.

${ }^{23}$ Wang, L. W.; Zunger, A. Phys. Rev. B 1996, 53 (15), 9579-9582.

${ }^{24}$ Hill, N. A.; Whaley, B. J. Chem. Phys. 1993, 99 (5), 3707-3715.

${ }^{25}$ Luttinger, J. M.; Kohn, W. Phys. Rev. 1955, 97 (4), 869-883.

${ }^{26}$ Luttinger, J. M. Phys. Rev. 1956, 102 (4), 1030-1041.

${ }^{27}$ Efros, Al. L. Phys. Rev. B, 1992, 46 (12), 7448-7458.

${ }^{28}$ Efros, Al. L.; Rosen, M.; Kuno, M.; Nirmal, M.; Norris, D. J.; Bawendi, M. Phys. Rev. B 1996, 54 (7), 4843-4856.

${ }^{29}$ Al. L. Efros and A. V. Rodina, Phys. Rev. B 1993, 47 (15), 10005-10007. 
${ }^{30}$ Nirmal, M.; Norris, D. J.; Kuno, M.; Bawendi, M. G. Efros, Al. L.; Rosen, M. Phys. Rev. Lett. 1995, 75 (20), 3728-3731. 


\section{Chapter 2. Controlled Synthesis of CdSe Nanorods}

\subsection{Introduction}

In the past decades, the synthesis of spherical CdSe nanocrystals has been so well developed $^{1,2,3}$ that the spherical CdSe nanocrystals can be made with excellent radius monodispersity, which allows for the systematic studies of quantum confinement in this model system.

In order to make crystals as small as a few nanometers, it is important to grow them in conditions away from equilibrium, because the formation of larger crystals are always more thermodynamically favored due to their lower surface energy. In practice, the nonequilibrium condition is guaranteed by maintaining the concentration of reactants higher than the solubility. This prevents the broadening of the size distribution due to the Oswald ripening ${ }^{3}$, where the smaller particles dissolve because of their higher surface energy while the larger ones grow even larger.

In our lab, the growth of nanoparticles with good size distribution is achieved by separating the nucleation and the growth. In order to have a good control over the size distribution of the CdSe nanocrystals, for example, the nucleation is initiated by a quick injection of precursors, followed by a quick decrease in the reaction temperature so that no nucleation happens afterwards and the growth of the nanocrystals takes over. 
Generally (nearly) spherical CdSe nanocrystals (aspect ratio < 1.2) are made by injecting the mixture of dimethyl cadmium and Se powder dissolved in tributylphosphine (TBP) into hot trioctylphosphine oxide (TOPO) that is protected in inert gases ${ }^{2}$. The thermal decomposition of the precursors results in the nucleation of the CdSe particles and the subsequent crystal growth. Typically the precursors are injected at $360{ }^{\circ} \mathrm{C}$ and then the temperature is lowered to $250{ }^{\circ} \mathrm{C}$ for crystal growth. Nanocrystals made in this way has wurtzite crystal structure, same as CdSe bulk crystals; and they are coated by the TOPO molecules to keep them from aggregation and to make them dispersible in organic solvents such as chloroform and toluene.

Recently Peng and Manna et $a l^{4}$ found that when a mixture of TOPO and hexylphosphonic acid (HPA) is used as the surfactant, rodlike CdSe nanocrystals can be made. The CdSe nanorods also have a wurtzite crystalline lattice, and are elongated in the $c$-crystallographic axis. However, the surfactant mixture of TOPO and HPA doesn't give us the capability to control the monodispersity or to tune the length and width of the nanorods. This is because the growth of the nanorods is so fast that it is very sensitive to subtle changes in the reaction conditions such as speed of precursor injection. As a result, nanocrystals with broad size distribution and even with different shapes are made ${ }^{5}$ On the other hand, it was observed that when HPA is replaced with a phosphonic acid with a longer alkyl chain, such as tetradcylphosphonic acid (TDPA), the growth speed of the nanocrystals is dramatically reduced, so much so that only nanorods with very small aspect ratio are made without further addition of the precursors during the growth process ${ }^{6}$. 


\subsection{Proposed Growth Mechanisms of CdSe Nanorods}

So far little is known about the microscopic growth mechanism of CdSe nanorods, mainly because of the lack of suitable experimental techniques. Based on the analysis of the products made by varying the growth conditions, a few observations have been made $^{5,6}$. First of all, rod growth is possible only when the monomer concentration is high enough, and even higher than what is required to avoid Oswald ripening. The monomers are believed to be some complexes of cadmium and selenium, which are formed at high temperature and may be very different from the stable species at room temperature, and their nature is not yet known. In order to maintain the monomer concentration for the nanorods to steadily grow along the $c$-axis, the presence of phosphonic acids is required. Secondly, when the monomers are depleted, unless more $\mathrm{Cd}$ and Se precursors are added, the nanorods will grow fatter and shorter so that eventually nearly spherical nanocrystals are formed. This suggests that the formation of elongated CdSe nanocrystals be kinetically favored while that of nearly spherical ones thermodynamically favored. Thirdly, the growth of the CdSe nanorods is unidirectional. This is based on the observation that when the amount of HPA in the surfactant mixture is higher than needed for nanorod growth, nanocrystals with unidirectional shapes such as pencil-, arrow-, or tree-like shapes are made ${ }^{5}$.

Based on the observations described above, so far two mechanisms have been proposed: selective adsorption ${ }^{5}$ and diffusion controlled growth mechanisms ${ }^{6}$. The selective adsorption mechanism is based on the different chemical affinities of phosphonic acids to the different faces on the nanocrystals, and thus the different energy 
of crystal faces in the presence of phosphonic acid molecules. According to this model, all crystal faces except $(00 \overline{1})$ is coated by the phosphonic acid molecules which slow down the adsorption of the monomers onto the faces, and therefore the nanocrystals grow unidirectionally to form rods. The diffusion controlled growth mechanism assumes the mass transport of the monomers to be the determining step of the nanocrystal growth, so that the concentration gradient of the monomers in the bulk solution and the stagnant solution around the nanorods determines their shape evolution. Both mechanisms emphasize the kinetic aspect of the nanorod growth, and accordingly assume that it is the activation barriers of the reactions that are affected by the presence of the phosphonic acid. The actual reactants in the reaction, or monomers, however, may also have been changed, considering the fact that by changing the alkyl chain in the phosphonic acids the growth rate of the nanorods can be greatly altered. This possibility is not yet considered in the currently existing models, and warrants further experimental studies.

\subsection{Synthesis of Tightly Controlled CdSe Nanorods}

In order to systematically study the size dependent properties of CdSe nanorods, we need to develop a simple method to synthesize them with well-controlled lengths and widths. The method we used is based on the methods described in sec. 2.1, i.e. dimethyl cadmium and Se dissolved in TBP are used as the precursors. Instead of using mixture of either HPA or TDPA and TOPO as the surfactant, however, we use a ternary mixture of HPA, TDPA and TOPO ${ }^{7}$. In addition, the $\mathrm{Cd}$ and Se precursors are added separately, instead of together, which was found to improve the size distribution of the nanorods produced $^{6}$. No matter what the mechanism may be, these modifications overcome the 
drawbacks of using a single phosphonic acid in the surfactant. By changing the relative amount of HPA and TDPA, we are able to tune the growth rate of the nanorods. Extraction of part of the reaction solution and subsequent quenching with a large excess of toluene at different time in the early stage of the reaction results in nanorods with different lengths $(<50 \mathrm{~nm})$ but almost the same diameter. As the reaction goes, concentrations of the monomers in the reaction solution are getting lower, and the nanorods grow fatter and shorter, as described earlier, and eventually becomes nearly spherical. However, if more precursors are added to the reaction, the growth of the nanocrystals along the $c$-axis continues without significant increase in the diameter, and thus nanorods longer than $50 \mathrm{~nm}$ can be made. The detailed synthesis procedure is described in the appendix.

In our study, the HPA/TDPA ratio is systematically changed from 0:1 to 3:1 while the total molar ratio of the phosphonic acids remains $20 \%$. The products are examined by transmission electron microscopy (TEM). Without adding more precursors after the crystal growth started, an HPA/TDPA ratio of 0:1 results in nanorods with large diameter (>4 $\mathrm{nm}$ ) but small aspect ratio $(<10)$, while increasing the amount of HPA in the mixture with fixed total molar ratio of the phosphonic acids and TOPO produces nanorods with smaller diameter but larger aspect ratio. When the HPA/TDPA ratio is higher than 3:1, the reaction loses control and nanoparticles with broad size and shape distributions are produced.

Figure 2.1 shows the electron transmission micrographs (TEM) of CdSe nanorods made with this method. The nanorods have diameters ranging from 3 to $7 \mathrm{~nm}$ and lengths from 3 to $70 \mathrm{~nm}$. Without any size selection performed on the samples after synthesis, the 
CdSe nanorods typically have $\sim 5 \%$ width distribution and $\sim 10 \%$ length distribution, as indicated by doing statistics on more than 200 particles in TEM images.
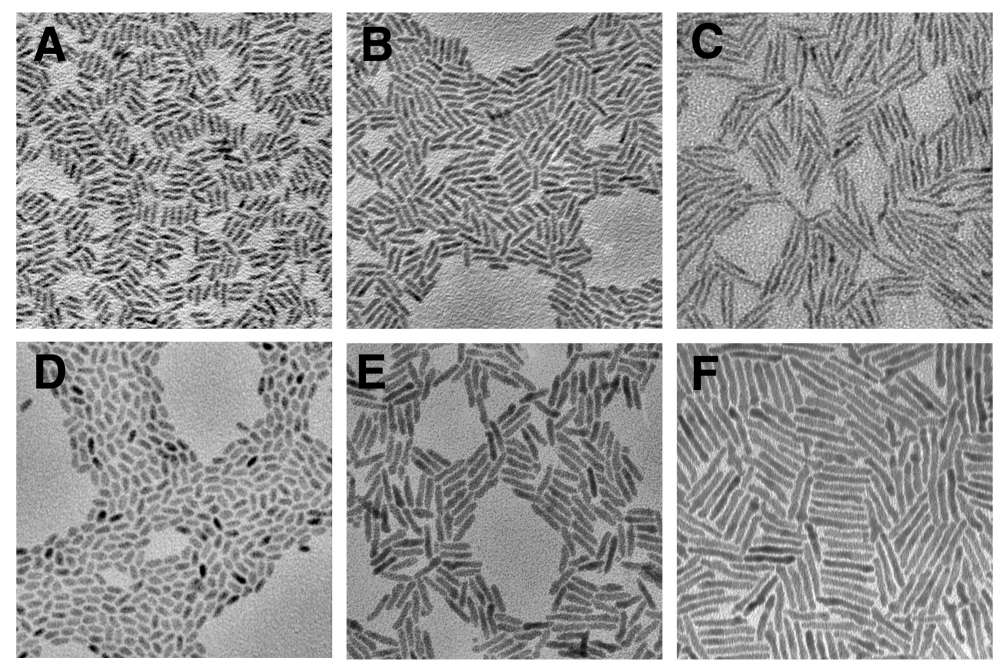

Fig.2.1 Transmission electron microscopy (TEM) images of CdSe nanorods made with HPA, TDPA and TOPO mixtures. 


\section{Appendix}

\section{Typical Procedures of the Synthesis of CdSe Nanorods}

A typical synthesis procedure is described as following. The whole reaction and the precursors are protected under argon.

Mixture of $3.536 \mathrm{~g}$ TOPO, $0.107 \mathrm{~g}$ HPA and $0.357 \mathrm{~g}$ TDPA (molar ratio HPA/TDPA=1/2) is heated to $150{ }^{\circ} \mathrm{C}$ under vacuum, and then to $360{ }^{\circ} \mathrm{C}$ under $\mathrm{Ar}$ protection. $0.50 \mathrm{~g} \mathrm{CdMe}_{2} / \mathrm{TBP}$ solution $(32.28 \%$ wt.) is added dropwise to the hot surfactant. The solution turns yellowish but clear after vigorous stirring. Then $2.50 \mathrm{~g}$ Se/TBP solution $(7.78 \%$ wt.) is injected into the hot solution, and crystal growth is initiated. The temperature is immediately lowered and kept at $250{ }^{\circ} \mathrm{C}$ for further crystal growth. At different time part of the solution is drawn out with a syringe and quenched with an excess of toluene, and nanocrystals with different sizes are thus obtained. UV/vis absorption spectra and photoluminescence (PL) are used to monitor the crystal growth, for nanocrystals have size dependent absorption and PL spectra. The global Stokes shift is used to estimate the aspect ratio of the nanorods (see. chapter 3).

In order to grow nanorods with longer length $(>50 \mathrm{~nm}), 1.0 \mathrm{ml}$ of the mixture of precursors $\left(0.41 \mathrm{~g} \mathrm{CdMe}_{2}, 0.12 \mathrm{~g}\right.$ Se and $7.72 \mathrm{~g}$ TBP) is further carefully added to the reaction solution dropwise, so that the monomer concentration is maintained high enough for the growth along the $c$-axis to continue. However, when the nanorods get longer the synthesis gets less reproducible, due to the branching caused by the polymorphism of CdSe. This is because CdSe nanocrystals can have both (hexagonal) wurtzite and (cubic) 
zinc blend structures, both of which have almost the same thermodynamic stability. The presence of four equivalent $\langle 111\rangle$ directions in the cubic segments in the nanorods results in branching as the nanocrystals grow along the $<111>$ directions. 


\section{Reference}

${ }^{1}$ Murray, C. B.; Norris, D. J.; Bawendi, M. G. J. Am. Chem. Soc. 1993, 115 (19), 87068715.

${ }^{2}$ Katari, J. E. B.; Colvin, V. L.; Alivisatos, A. P. J. Phys. Chem. 1994, 98 (15), 41094117.

${ }^{3}$ Peng, X. G.; Wickham, J.; Alivisatos, A. P. J. Am. Chem. Soc. 1998, 120 (21), 53435344.

${ }^{4}$ Peng, X.; Manna, L.; Yang, W.; Wickham, J.; Scher, E.; Kadavanich, A.; Alivisatos, A. P. Nature 2000, 404 (6773), 59-61.

${ }^{5}$ Manna, L.; Scher, E.; Alivisatos, A. P. J. Am. Chem. Soc. 2000, 122 (51), 12700-12706.

${ }^{6}$ Peng, Z. A.; Peng, X. J. Am. Chem. Soc. 2001, 123 (7), 1389-1395.

${ }^{7}$ Hu, J.; Li, L.-S.; Yang, W.; Manna, L.; Wang, L.-W.; Alivisatos, A. P. Science, 2001, 292 (5524), 2060-2063.

${ }^{8}$ Li, L.-S.; Hu, J.; Yang, W.; Alivisatos, A. P. Nano Lett. 2001, 1 (7), 349-351. 


\section{Chapter 3. Optical Properties of CdSe Nanorods}

\subsection{Introduction}

It has been well known that the broken spherical symmetry of the atoms alters the polarization of their light emission ${ }^{1}$. Examples are Zeeman effect and Stark effect in atomic spectra. Similarly, it can also be expected that shape anisotropy of nanocrystals should result in optical anisotropy. This has indeed been observed in metallic nanocrystals ${ }^{2}$. For example, when Au nanocrystals get elongated, the surface plasmon resonance becomes non-degenerate and thus splits into transverse and longitudinal modes. Furthermore, the "lightening rod" effect caused by the geometrical anisotropy also enhances the anisotropy in their optical properties. For metallic nanocrystals, continuum models ${ }^{34}$ without any consideration on their internal bonding structures are sufficient to explain and predict their optical properties. For semiconductor nanocrystals, however, the bonding and lattice structures ${ }^{5}$, in addition to the geometrical factor ${ }^{6}$, have to be considered due to the localization of the carriers.

Even in spherical CdSe nanocrystals, 2-dimensional polarization of the photoluminescence in the $a-b$ crystallographic plane has been theoretically predicted ${ }^{7} 89$ and experimentally verified ${ }^{10}$, due to the uniaxial wurtzite crystal structure, i.e. the nonvanishing crystal-field splitting in the effective mass approximation described in chapter 1. The extrapolation (even though not necessarily valid) of the result for the slightly elongated CdSe nanocrystals (Eq. 1.2 in Chapter 1) suggests that when the ellipticity of 
the nanocrystals $\mu$ increases, the shape correction should be able to compensate the crystal field splitting and even change the order of the lowest hole states, and subsequently the polarization of the photoluminescence. In this chapter, the tunability and the polarization of the photoluminescence of the CdSe nanorods are discussed, as well as the explanation to the observed polarization by a semi-empirical pseudopotential calculation.

\subsection{Size dependence of Photoluminescence Spectra of CdSe Nanorods}

The capability to independently tune the diameter and length of CdSe nanorods offers us an opportunity to study the dependence of their optical properties on their sizes. Not surprisingly, the wavelength of the photoluminescence from the nanorods can be tuned by varying either their width or length. Figure 3.1 shows the photoluminescence spectra of the nanorods with the same width but four different lengths. The samples were dispersed in toluene, and their photoluminescence spectra were measured on a commercial Spex $1682 \quad 0.22 \mathrm{~m}$ fluorimeter at room temperature. The spectral resolution for the

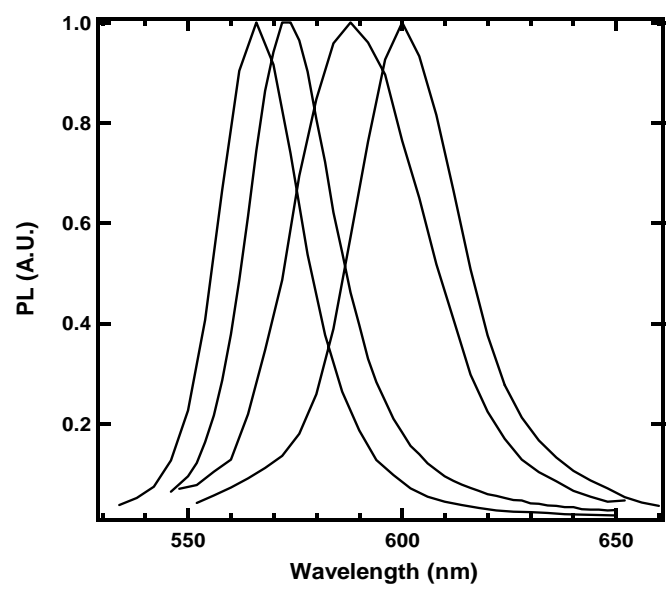
photoluminescence spectra is $4 \mathrm{~nm}$. All the samples were excited at $450 \mathrm{~nm}$, which is far shorter than their absorption edges so

Fig. 3.1 Photoluminescence spectra of 3.7 ( \pm 0.2) $\mathrm{nm}$ wide $\mathrm{CdSe}$ nanorods with lengths of 9.2, 11.5, 28.0 and $37.2 \mathrm{~nm}$, respectively (from left to right), excited at $450 \mathrm{~nm}$. 
that size-selective excitation is avoided. The room temperature quantum efficiency of the rods is typically $5-10 \%$. By changing only the length of CdSe nanorods, we can tune the wavelength of their emission over the same range as in spherical CdSe nanocrystals.

In Fig. 3.2, we show the band gap variation versus width and length at room temperature for nanorod samples, with aspect ratio ranging from 1 to $12^{11}$. The original data are also tabulated in the appendix. As expected from quantum confinement considerations, the general tendency is that the emission shifts to lower energy with an increase in either width or length. The data are fit with a polynomial in $1 /$ length $(1 / \mathrm{L})$, 1/width (1/W) and aspect ratio (L/W). The surface of best fit obtained is 1.8563 $2.0835 / \mathrm{L}^{2}+4.5507 / \mathrm{W}^{2}-0.0018(\mathrm{~L} / \mathrm{W})^{2}+0.0001(\mathrm{~L} / \mathrm{W})^{3}+10.5824 / \mathrm{L}^{3}-0.3833 / \mathrm{W}^{3}$. The standard deviation of this fit is $\sim 30 \mathrm{meV}$, which is only slightly greater than the thermal
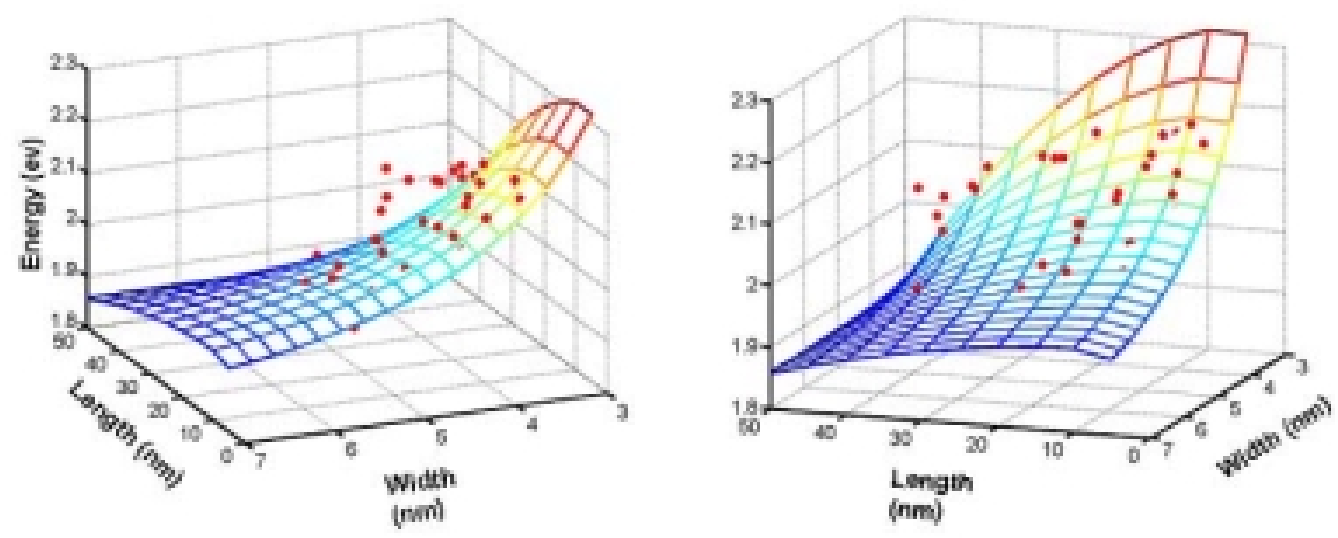

Fig. 3.2 Band gap of CdSe nanorods versus length and width viewed from two different angles. The meshes are the best fit described in the text.

energy at room temperature. The polynomial fit is provided for the convenience of the reader, but has no direct physical significance. On the surface we can see a slight increase 
in band gap $(<20 \mathrm{meV})$ for rods shorter than $10 \mathrm{~nm}$. This is due to uncertainty in both the experiments and the fitting, thus cannot be extrapolated to disk-like nanocrystals.

One distinct feature of figure 3.2 is that the slope of the band gap with respect to the width of the nanorods is much larger than that to the length. This indicates that the band gap is mainly determined by the lateral confinement, which plays an important role even when rods are very long. This can be further justified by the fact that the slope of the band gap with respect to width is almost the same for the rods with the same width but very different lengths. A quantitative description of the band gap variation for $\mathrm{CdSe}$ nanorods is not yet available. However, it can be simply understood with the uncertainty principle. Here the sensitivity of the band gap dependence on the dimension in different directions can be roughly measured by the uncertainty in the determination of the translational momentum, which scales with $\sim 1 /$ length (or width).

\subsection{Dependence of the Global Stokes Shift on the Aspect Ratio of CdSe Nanorods}

The global Stokes shift (or the nonresonant Stokes shift) of the nanocrystals is defined to be the energy difference between the red-most band-edge absorption peak and the photoluminescence peak, when the nanocrystals are excited by photons with energy far above the absorption edge (to avoid the spectral size selection). For nanocrystals with very narrow size distribution $(<5 \%)$, the global Stokes shift is determined by the energy relaxation mechanism following the optical excitation. For nearly spherical CdSe nanocrystals, it was found to be monotonically decreasing with increasing particle size, which has been successfully explained by the "dark exciton" model ${ }^{9}$. 
The global Stokes shift of CdSe nanorods shows a peculiar behavior ${ }^{12}$. Here, for a given width, it is not monotonic with increasing aspect ratio. Figure 3.3 shows the experimental values for nanorods with $\sim 3.9 \mathrm{~nm}$

width. When the aspect ratio is smaller than $\sim 3$ the Stokes shift decreases with increase in length; while it increases with length for aspect ratio larger than 3. Since the nanorods have a width distribution narrower than $5 \%$ and length distribution less than $10 \%$ consistently, the size distribution does not alter the trend in the change of the Stokes shift, as has been shown in spherical

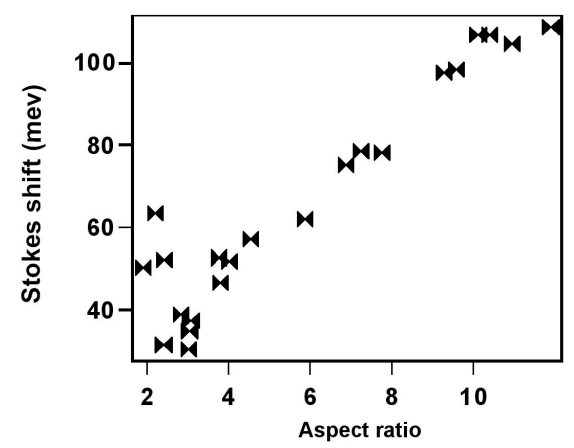
Fig. 3.3. Experimental results of the global Stokes shift versus aspect ratio for nanorods with diameters of $3.9 \pm 0.2 \mathrm{~nm}$. Rods with different widths show similar nanoparticles 9 . Nanorods with different widths trends. show similar trends, and therefore the Stokes shift can be used as a rough estimation on the aspect ratio of the nanorods during synthesis (chapter 2, appendix), while the first absorption peak can be used to estimate their width. This suggests there may indeed be a crossover in the electronic states, as expected from the qualitative argument of the competition between the ellipticity and the crystal field splitting in the wurtzite structure (chapter 1).

\subsection{Linear Polarization of the Photoluminescence of CdSe Nanorods}

As discussed in Sec. 3.1, it is well expected that the photoluminescence of $\mathrm{CdSe}$ nanorods should have different polarization properties from that of nearly spherical nanocrystals, as a result of the compensation of the crystal field splitting in the wurtzite 
structure by the ellipticity. With increasing aspect ratio, the fluorescence emitted by the nanocrystals should evolve from 2-dimensional polarized in the $a$ - $b$ crystallographic plane to linearly polarized along the $c$-axis. It was first proved by the ensemble photoluminescence measurement of CdSe nanorods embedded in a stretched polymer ${ }^{13}$. However, it was not until the advent of the synthetic method able to tightly control the width and the length of the nanorods that the systematic study of the polarization evolution becomes possible.

We performed the single molecule spectroscopy on the CdSe nanorods with different lengths and widths ${ }^{12}$. The experimental setup is a confocal fluorescence microscope similar to the one described in reference14. A dilute solution of the nanoparticles in chloroform was spin-cast onto a quartz substrate, and an atomic force microscope was used to make sure that individual rods are separated by at least $0.5 \mu \mathrm{m}$. We used a circularly polarized $\mathrm{Ar}^{+}$ion laser $(488 \mathrm{~nm})$ to excite the nanocrystals (to avoid the possible effect of excitation polarization), and a beam-displacing crystal to split the luminescence light into two perpendicularly polarized beams. The two split images are 2 $\mathrm{cm}$ apart and can be simultaneously recorded with a single CCD camera. By rotating the beam displacing crystal, polarizations along any pair of orthogonal directions can be determined. Images of luminescence from a single nanorod with 10:1 aspect ratio (Fig. 3.4A) demonstrate the change with detection angle of the relative intensity along two perpendicular polarization directions. The polarization factor $r$ for this rod sample is determined to be $86 \%$ by fitting the intensity ratio $r=\left(\mathrm{I}_{/ /} \mathrm{I}_{\perp}\right) /\left(\mathrm{I}_{/ /}+\mathrm{I}_{\perp}\right)$ versus detection angle with a sinusoidal function (filled circle in Fig. 3.4B). In comparison, the polarization factor of spherical nanocrystals is usually less than $10 \%$ (filled triangles in 
Fig. 3.4B). We have measured samples with aspect ratio from 1:1 to $30: 1$ at room temperature. The polarization factor changes rapidly from nearly zero to $\sim 70 \%$ (e.g.,

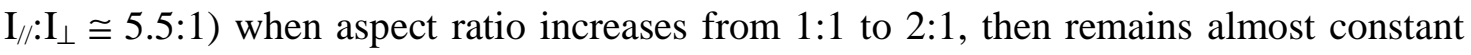
afterwards (Fig. 3.4C). At low temperature, polarization factors of emission from spherical quantum dots take random values between 0 and 1 because of the "dark" $c$-axis and the random orientation of the dots on the substrate ${ }^{10}$. However, the statistics from more than 40 spherical quantum dots measured at room temperature show that the average polarization factor is $<10 \%$. Because we used an objective lens with a large numerical aperture (e.g., 1.4) that depolarizes light, the upper limit of polarization factor that our experimental setup can obtain is about $80 \%$.

The observation of the linear polarization of porous Si photoluminescence has been reported, and was explained by a dielectric model which only considers the anisotropic local field factors, with the assumption that the porous $\mathrm{Si}$ consists of aggregates of elongated and flattened Si nanoparticles ${ }^{6}$. The application of this model to CdSe nanorods is shown with the dotted line in figure 3.4 C. It is obvious that this model cannot explain the abrupt jump in the polarization factor for small aspect ratio nanorods. 


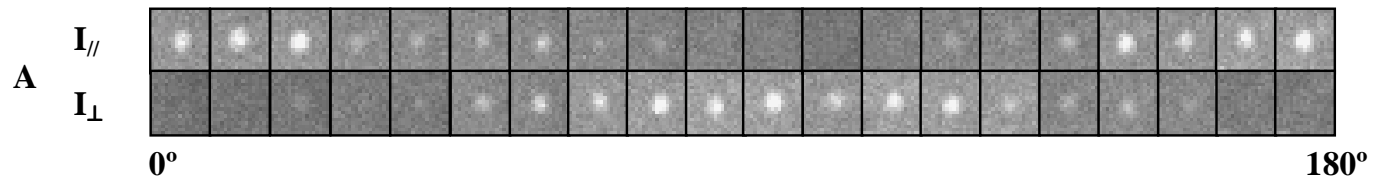

B

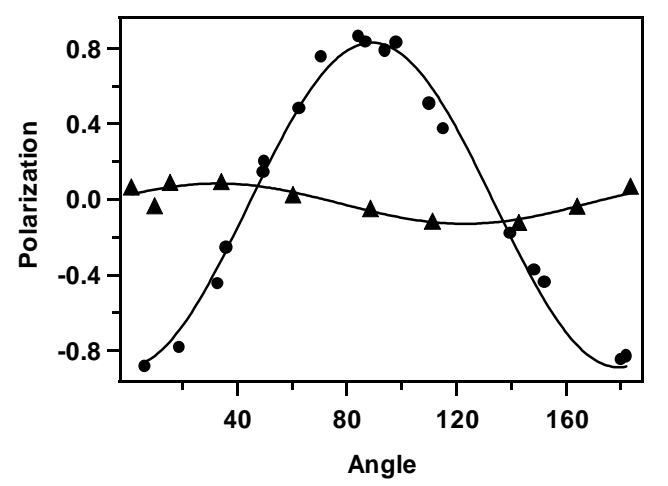

$\mathbf{C}$

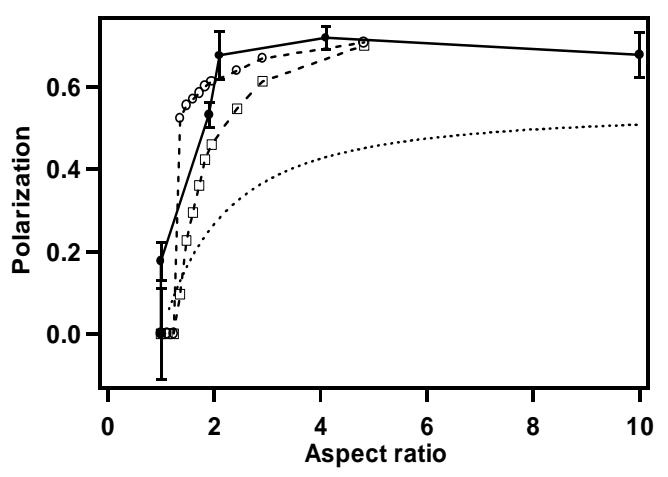

Fig. 3.4. Luminescence polarization measurements from individual nanorods at room temperature. (A) Luminescence images of a single rod simultaneously recorded in two perpendicular polarization directions at detection angles changing from $0^{\circ}$ to $180^{\circ}$. The rods have an aspect ratio of 10:1. (B) The intensity ratio $\mathrm{r}=\left(\mathrm{I}_{/ /} \mathrm{I}_{\perp}\right) /\left(\mathrm{I}_{/ /}+\mathrm{I}_{\perp}\right)$ calculated from the luminescence images in A (filled circles) and fitted with sinusoidal function (solid line) to give a polarization factor of 0.86 . The data from spherical dots (filled triangles) show much smaller polarization factors. (C) Polarization factor vs. aspect ratio. The filled circles with error bars are from experiments, unfilled circles from empirical pseudopotential calculations for $0 \mathrm{~K}$, and squares from empirical pseudopotential calculations for room temperature. Each experimental data point was obtained by measuring more than 40 individual rods. The lines simply connect data points, and the dotted line is from the fitting based on the dielectric model proposed in ref. 6 


\subsection{Evolution of the Electronic Structure of CdSe Nanorods with Aspect Ratio - Theoretical Study}

In order to explain the evolution of the photoluminescence polarization and the nonmonotonic behavior observed in the global Stokes shift of CdSe nanorods, a nonperturbative method has to be used, due to the large aspect ratio of the nanorods we have studied experimentally.

The electronic structure of CdSe nanorods is calculated using a semiempirical pseudopotential method ${ }^{15,16}$. Based on ab initio local density approximation (LDA) calculations on bulk CdSe, approximate screened atomic spherical potentials that reproduce the bulk LDA potential for $\mathrm{Cd}$ and $\mathrm{Se}$ atoms are developed. Small modifications are made to the screened atomic potentials to correct the LDA band gap errors, and to fit the bulk CdSe band structure. The resulting semiempirical pseudopotentials $v^{\alpha}(r)$ 's [ $\alpha$ stands for atomic types, $\mathrm{Cd}$ or Se] are used to construct the single particle Hamiltonian and the corresponding Schrödinger equation:

$\left\{-\frac{1}{2} \nabla^{2}+\sum_{\alpha, R} v^{\alpha}(|r-R|)+\hat{V}_{\text {nonlocal }}(r)\right\} \psi_{i}(r)=\varepsilon_{i} \psi_{i}(r)$,

where $R$ 's are the atomic positions, and $\hat{V}_{\text {nonlocal }}(r)$ the non-local part of the pseudopotential, which is the same as that in the LDA ab initio pseudopotentials. The wavefunctions $\left\{\psi_{i}(r)\right\}$ are expressed in a planewave basis set, using a cutoff of 6.8 Rydberg for the planewave kinetic energy. Spin-orbit coupling is included in the non- 
local potential $\hat{V}_{\text {nonlocal }}(r)$. As a result, the wavefunctions $\left\{\psi_{i}(r)\right\}$ are spinors with spin up and spin down components.

In the calculation, a rod shape is generated from the bulk wurtzite CdSe with the long axis along the crystallographic $c$-axis. To change the aspect ratio of the rod, we start from a spherical dot with the desired diameter and insert a cylindrical segment along the $c$-axis. We have removed surface $\mathrm{Cd}$ and $\mathrm{Se}$ atoms with only one bond connected to the rest of the rod. As a result, the numbers of $\mathrm{Cd}$ and Se atoms may not be the same. However, as we are not doing total energy calculations, such a slight nonstoichiometry does not cause any problem. The surface $\mathrm{Cd}$ and $\mathrm{Se}$ atoms are passivated using different hypothetical ligand potentials. This is in part to simulate the experimental situation of surfactant passivation, and in practice to remove surface dangling bond states from the band gap. The detail of this passivation is described in ref. 17. In terms of calculation, this is to add a few additional atomic types $\alpha$ and their corresponding passivation potentials $v^{\alpha}(r)$ in the above Schrödinger equation.

The Schrödinger equation is solved for eigenstates $\left\{\psi_{i}(r)\right\}$ near the band edge. This is done with the folded spectrum method (FSM) ${ }^{18}$. Only a few levels near the band edge are solved for the rods of all sizes. As a result, this method scales linearly to the size of the system. A parallel program (Escan) is developed based on FSM, which uses a parallel FFT, and a real space implementation of the non-local potential in the above equation $^{19}$. The calculations are carried out using the Cray T3E parallel computer in National Energy Research Scientific Computing Center (NERSC). 
The above approach has been used extensively to study the electronic structures of CdSe quantum dots ${ }^{17}{ }^{19}$. These include: band gap vs. the dot size, optical absorption spectra, higher excited states, Coulomb and exchange interactions, multi-exciton fine structures, etc. Here we apply it to CdSe nanorods in particular to study the changes of the electronic structures as the aspect ratio of the rods changes.

Although the size dependence of the band gap of the nanorods as shown in Sec. 3.2, has not been quantitatively reproduced, the pseudopotential calculation has confirmed the crossover of the energy levels of the highest occupied electronic states, as shown in figure 3.5 for $3.0 \mathrm{~nm}$ wide nanorods. Before the crossing point, the electronic states with predominantly Se $4 p_{x}, y$ components $\left(F_{z}= \pm 3 / 2\right.$ states in chapter 1$)$ have higher energy than that of states that are mainly $4 p_{z}\left(F_{z}= \pm 1 / 2\right.$ states $)$. The electronic energy levels of these states all increase with increasing size. The $4 p_{z}$ orbital has greater momentum projected onto the $c$-axis of the crystal compared to $4 p_{x, y}$ orbitals, so the energy levels with more $4 p_{z}$ component is more sensitive to the change in the rod length ${ }^{12}$. As a result. these states exchange positions relative to one another at an aspect ratio greater than 1.25. Calculations for 2.1 and $3.8 \mathrm{~nm}$ wide rods show similar results and the crossing points are 1.25 and 1.36 respectively.

This calculation also qualitatively reproduces the turning point in the global Stokes shift (figure 3.6), in spite of the deviation from the exact value. In the calculation, the absorption spectra are calculated as the sum of all of the possible transitions from the highest four occupied states to the lowest four unoccupied states (not shown in fig. 3.5) weighed by their oscillator strengths, allowing a Gaussian distribution of lengths with a $10 \%$ standard deviation. The first absorption maximum from the red edge is considered to 
be the first absorption peak. Its position only slightly changes for nanorods with the same

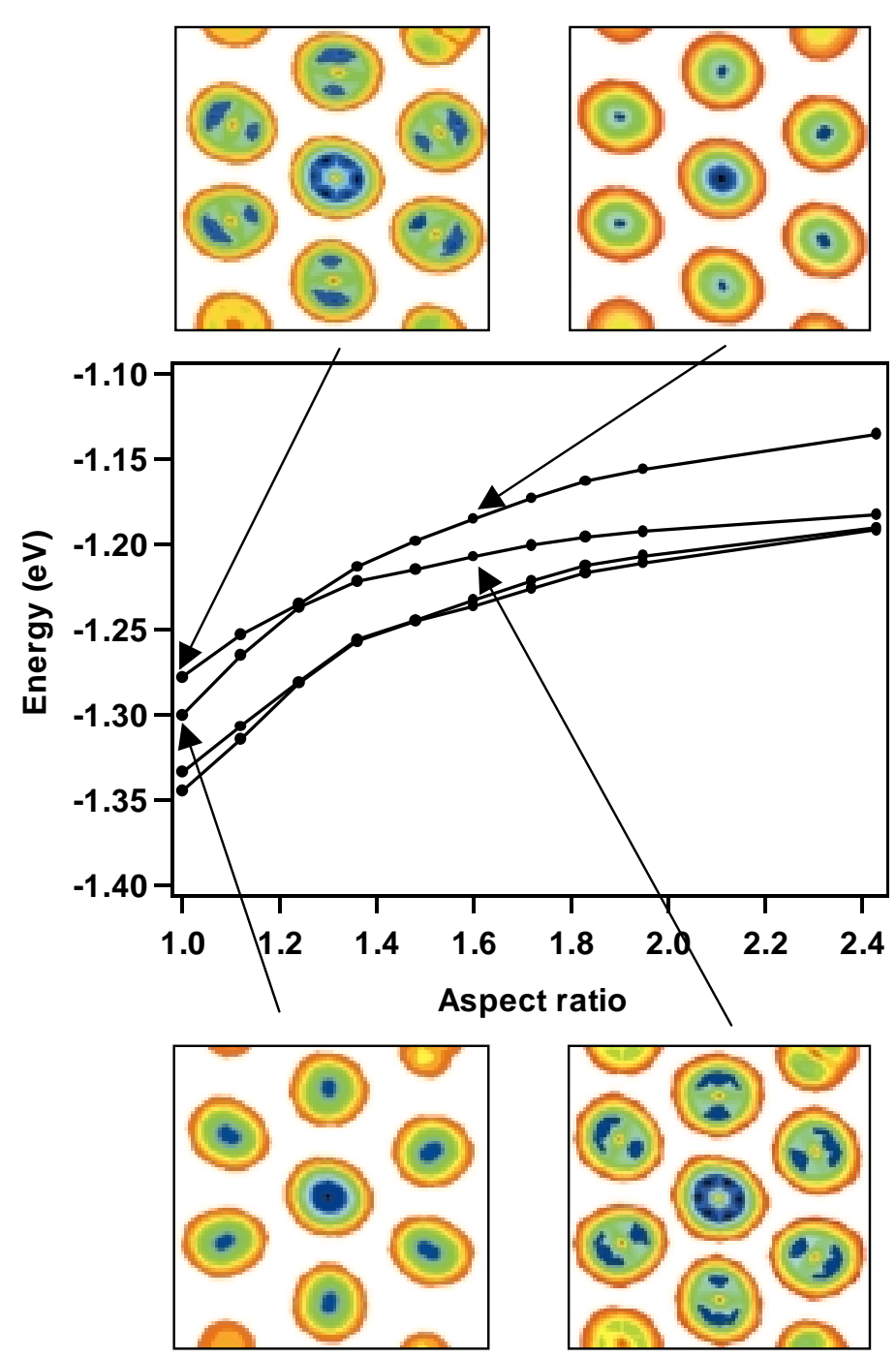

Fig. 3.5. The four highest occupied electronic states of 3.0 nm diameter CdSe nanorods calculated with an empirical pseudopotential method with different aspect ratios. The two highest energy levels have a crossing at an aspect ratio around 1.25. Insets: Contour plots of the two highest occupied states for rods with an aspect ratio of 1.00 and 1.60 , respectively. They show the projection of electron density around some Se atoms in a plane, with density increasing from red to blue. This plane intersects the rod in the middle, perpendicular to the long axis ( $c$ axis). The crossing over of the predominantly $\quad \mathrm{p}_{x, y} \quad$ and predominantly $\quad \mathrm{p}_{z}$ levels $v s$. aspect ratio can be seen.

width but different lengths, for it comes from the superposition of a few transitions, while 
the photoluminescence energy can change by up to $50 \mathrm{meV}$, therefore the Stokes shift roughly coincides with the change in the energy of the highest occupied electronic states.

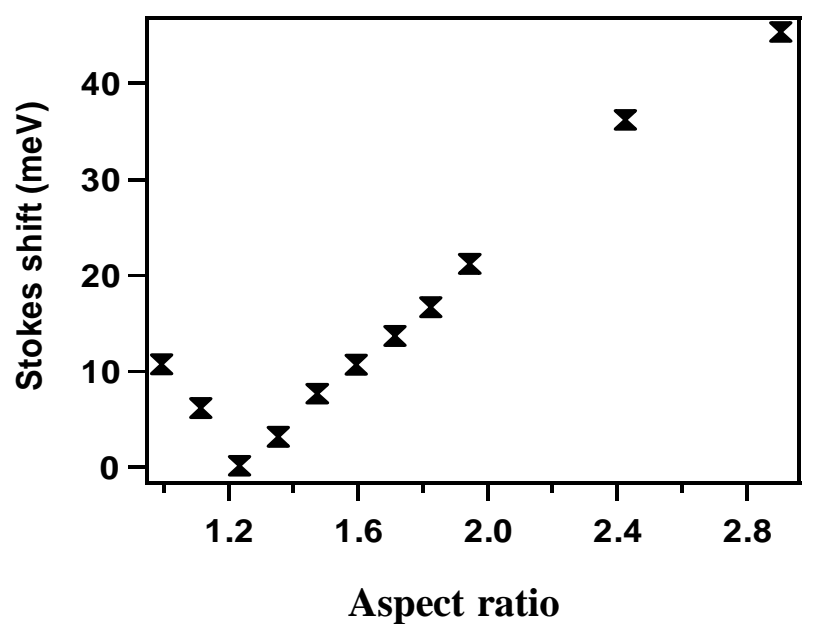

Fig. 3.6 Calculated global Stokes shift versus aspect ratio for 3.0-nm-wide nanorods. This is qualitatively the same as the experimental results shown in figure 3.3 .

\subsection{Summary}

We have shown that the CdSe nanorods have size-dependent luminescence energy, like spherical ones. In addition, they have linearly polarized photoemission, as a result of the level crossing in their highest occupied electronic states with increasing aspect ratio. This level crossing can be qualitatively understood as the competition between the crystal field splitting in the wurtzite structure and the energy shift caused by the ellipticity of the nanorods, and has been semi-quantitatively explained by a semi-empirical pseudopotential calculation.

Because of the simple synthetic method for making CdSe nanorods, as well as their novel properties, it is advantageous to use the nanorods for many applications instead of the nearly spherical particles without sacrificing the size-dependent properties. In 
addition, the geometrical anisotropy of the nanorods greatly facilitates the assembly of these nanoparticles, as will be described in later chapters.

\section{Appendix}

\section{A. The Band Gap of CdSe Nanorods}

\begin{tabular}{|c|c|c|c|c|c|c|c|}
\hline Length (nm) & $\begin{array}{r}\text { Width } \\
\text { (nm) }\end{array}$ & $\begin{array}{r}\mathrm{PL}(\mathrm{eV}) \\
295 \mathrm{~K}\end{array}$ & $\begin{array}{r}\mathrm{PL}(\mathrm{eV}) \\
7 \mathrm{~K}\end{array}$ & $\begin{array}{r}\text { Length } \\
(\mathrm{nm})\end{array}$ & $\begin{array}{r}\text { Width } \\
(\mathrm{nm})\end{array}$ & $\begin{array}{r}\mathrm{PL}(\mathrm{eV}) \\
295 \mathrm{~K}\end{array}$ & $\begin{array}{r}\mathrm{PL}(\mathrm{eV}) \\
7 \mathrm{~K}\end{array}$ \\
\hline $11.0( \pm 0.7)$ & 3.2 & 2.20 & & $8.7( \pm 0.9)$ & 4.3 & 2.07 & \\
\hline $37.8( \pm 3.0)$ & 3.3 & 2.08 & & $16.4( \pm 2.0)$ & 4.3 & 2.08 & \\
\hline $43.1( \pm 3.2)$ & 3.4 & 2.03 & & $8.6( \pm 1.0)$ & 4.4 & 2.10 & \\
\hline $28.0( \pm 2.2)$ & 3.5 & 2.11 & & $31.5( \pm 3.3)$ & 4.4 & 1.98 & \\
\hline $38.5( \pm 4.4)$ & 3.5 & 2.05 & & $15.3( \pm 0.8)$ & 4.5 & 2.10 & \\
\hline $11.5( \pm 0.8)$ & 3.6 & 2.17 & 2.20 & $19.8( \pm 2.0)$ & 4.6 & 2.02 & \\
\hline $22.1( \pm 1.8)$ & 3.6 & 2.16 & & $19.8( \pm 1.2)$ & 4.6 & 1.97 & \\
\hline $7.6( \pm 0.8)$ & 3.7 & 2.16 & & $12.4( \pm 1.3)$ & 4.8 & 2.03 & \\
\hline $9.2( \pm 0.7)$ & 3.7 & 2.19 & & $19.4( \pm 1.4)$ & 4.8 & 2.03 & \\
\hline $26.1( \pm 3.2)$ & 3.7 & 2.12 & 2.17 & $40.4( \pm 3.7)$ & 4.8 & 1.93 & \\
\hline $28.8( \pm 4.8)$ & 3.7 & 2.12 & & $18.4( \pm 2.0)$ & 4.9 & 2.06 & \\
\hline $8.6( \pm 0.8)$ & 3.8 & 2.12 & & $18.9( \pm 2.1)$ & 4.9 & 2.06 & 2.09 \\
\hline $37.2( \pm 4.0)$ & 3.9 & 2.07 & 2.10 & $12.0( \pm 1.4)$ & 5.1 & 1.99 & \\
\hline $44.3( \pm 4.0)$ & 3.9 & 2.06 & 2.10 & $11.4( \pm 1.2)$ & 5.2 & 2.00 & \\
\hline $9.7( \pm 0.7)$ & 4.0 & 2.12 & & $22.2( \pm 2.2)$ & 5.2 & 2.00 & \\
\hline $11.6( \pm 1.0)$ & 4.0 & 2.18 & 2.23 & $40.8( \pm 4.2)$ & 5.3 & 1.90 & \\
\hline $41.3( \pm 6.8)$ & 4.0 & 2.02 & & $18.2( \pm 1.5)$ & 5.4 & 2.00 & \\
\hline $12.7( \pm 1.0)$ & 4.1 & 2.15 & & $8.5( \pm 1.0)$ & 5.5 & 1.95 & \\
\hline $13.4( \pm 1.1)$ & 4.1 & 2.13 & & $18.4( \pm 1.8)$ & 5.5 & 1.96 & 2.02 \\
\hline $16.9( \pm 2.3)$ & 4.1 & 2.04 & & $23.6( \pm 2.9)$ & 5.5 & 1.97 & \\
\hline $40.2( \pm 4.0)$ & 4.1 & 2.00 & & $14.0( \pm 1.2)$ & 6.2 & 1.94 & \\
\hline $16.5( \pm 2.4)$ & 4.2 & 2.06 & & $17.6( \pm 1.0)$ & 6.4 & 1.93 & \\
\hline $20.2( \pm 2.0)$ & 4.2 & 2.02 & 2.05 & & & & \\
\hline
\end{tabular}




\section{Reference}

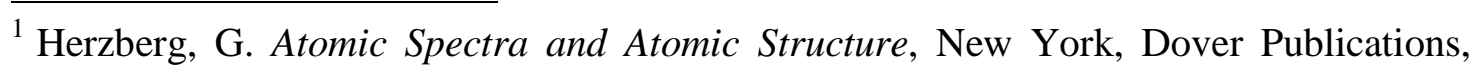
1945.

${ }^{2}$ El-Sayed, M. A. Acct. Chem. Res. 2001, 34 (4), 257-264.

${ }^{3}$ Mie, G. Ann. Phys. 1908, 25, 329.

${ }^{4}$ Gans, R. Ann. Phys. 1915, 47, 270.

${ }^{5}$ McIntyre, C. R.; Sham, L. J. Phys. Rev. B 1992, 45 (16), 9443-9446.

${ }^{6}$ Kovalev, D.; Benchorin, M.; Diener, J.; Koch, F.; Efros, A. L.;Rosen, M.; Gippius, N.

A.; Tikhodeev, S. G. Appl. Phys. Lett. 1995, 67 (11), 1585-1587.

${ }^{7}$ Efros, Al. L. Phys. Rev. B 1992, 46 (12), 7448-7458.

${ }^{8}$ Efros, Al. L.; Rodina, A. V. Phys. Rev. B 1993, 47 (15), 10005-10007.

${ }^{9}$ Efros, Al. L.; Rosen, M.; Kuno, M.; Nirmal, M.; Norris, D. J.; Bawendi, M. Phys. Rev. B 1996, 54 (7), 4843-4856.

${ }^{10}$ Empedocles, S. A.; Neuhauser, R.; Bawendi, M. G. Nature 1999, 399 (6732), 126-130.

${ }^{11}$ Li, L.-S.; Hu, J.; Yang, W.; Alivisatos, A. P. Nano Lett. 2001, 1 (7), 349-351.

${ }^{12}$ Hu, J.; Li, L.-S.; Yang, W.; Manna, L.; Wang, L.-W.; Alivisatos, A. P. Science, 2001, 292 (5524), 2060-2063.

${ }^{13}$ Peng, X.; Manna, L.; Yang, W.; Wickham, J.; Scher, E.; Kadavanich, A.; Alivisatos, A. P. Nature 2000, 404 (6773), 59-61.

${ }^{14}$ Ha, T.; Laurence, T. A.; Chemla, D. S.; Weiss, S. J. Phys. Chem. B 1999, 103 (33), 6839-6850.

${ }^{15}$ Wang, L. W.; Zunger, A. Phys. Rev. B 1995, 51 (24), 17398-17416. 
${ }^{16}$ Hu, J.; Wang, L.-W.; Li, L.-S.; Yang, W.; Alivisatos, A. P. J. Phys. Chem. B 2002, 106 (10), 2447-2452.

${ }^{17}$ Wang, L. W.; Zunger, A. Phys. Rev. B 1996, 53 (15), 9579-9582.

${ }^{18}$ Wang, L. W.; Zunger, A. J. Chem. Phys. 1994, 100 (3), 2394-2397.

${ }^{19}$ Canning, A.; Wang, L. W.; Williamson, A.; Zunger, A. J. Comp. Phys. 2000, 160 (1), $29-41$. 


\section{Chapter 4. Permanent Electric Dipole Moment of CdSe Nanorods ${ }^{1}$}

\subsection{Introduction}

Although nanocrystals consist of hundreds and even thousands of atoms, it is often instructive to consider each of them to be an individual molecule. Thus it is of great interest to study the dielectric properties such as electric permanent dipole moment and polarizability of these giant molecules, in order to understand their interactions with external environment and with each other. In contrast to their well-studied optical and electronic properties, however, the dielectric properties of CdSe nanocrystals have seldom been investigated. Especially, in the few prior studies on their permanent electric dipole moment, contradictory results have been reported. On one hand, theoretical analyses have suggested a permanent dipole moment along the $c$-crystallographic axis due to the lack of inversion symmetry in wurtzite structure ${ }^{234}$, and it should scale linearly with the volume; on the other hand, dielectric dispersion measurement performed on concentrated solutions of spherical $\mathrm{CdSe}^{56}$ nanocrystals have found a permanent dipole moment linear to the diameter of the nanocrystals within the experimental error. The authors proposed ${ }^{6}$ a surface localized charge model to explain the experimental results, assuming only one pair of trapped charges present on the surface of the spherical nanocrystals and their distance linear to the diameter of the nanocrystals. They further concluded that the contribution of the non-centrosymmetric lattice is negligible in the size range studied. 
However, the surface charge model does not address the direction of the permanent dipole moment, nor does the dielectric dispersion measurement itself, because it does not distinguish the uniaxial nature of the spherical nanocrystals. The anisotropic shape of the CdSe nanorods gives us an opportunity to answer this question. Furthermore, since the volume of the nanorods is much larger than that of the spherical nanocrystals that have been studied in the dielectric dispersion measurement, the lattice contribution to the permanent dipole moment may be significant enough to be measured experimentally.

We perform transient electric birefringence (TEB) measurement on dilute solution of CdSe nanorods with various widths and lengths by taking advantage of the anisotropy in both the static and the optical dielectric constant of the nanorods caused by their geometrical anisotropy. The former makes the nanorods align more easily under static electric fields, while the latter results in birefringence once the nanorods are aligned. From the sign of the birefringence we determine the orientation of the nanorods under the electric field; and from the transient behavior of the birefringence we obtain the relationship between the permanent dipole moments and the sizes of the nanorods, which reveals the origin of the dipole moment for nanocrystals in this size regime. 


\subsection{Electric Birefringence Measurement}

\subsubsection{Basic principle}

Electric birefringence measurement, also know as Kerr effect, is a conventional way to measure the response of a system to an external field, and has been widely applied in the determination of dielectric properties of both small molecules and macromolecules and even viruses ${ }^{789}$. The electric birefringence of a medium is given by

$$
\Delta n=n_{\|}-n_{\perp}=B \lambda E^{2}
$$

where $\lambda$ the in vacuo wavelength of the light, $n_{\|}, n_{\perp}$ the refractive indices of the medium for the light of the given wavelength respectively along and perpendicular to the applied electric field, $E$ the strength of the applied electric field and $B$ the Kerr constant. For a solution of rigid particles with revolute ellipsoidal shape dispersed in an isotropic solvent, the relationship between the birefringence and the properties of the ellipsoid is given by ${ }^{10}$ 11

$$
\Delta n=\frac{2 \pi\left(g_{z}-g_{x}\right)}{15} \rho E^{2}\left[\left(\frac{\mu_{z}^{\prime}}{k T}\right)^{2}-\left(\frac{\mu_{x}^{\prime}}{k T}\right)^{2}+\frac{\alpha_{z}-\alpha_{x}}{k T}\right]
$$

where $k$ the Boltzmann constant, $\mathrm{T}$ the temperature, $g_{z, x}$ the optical polarizability per unit volume of the ellipsoids along and perpendicular to their unique axis respectively, $\alpha_{z, x}$ their static polarizability, and $\mu_{z, x}^{\prime}$ the screened permanent dipole moment of the ellipsoid in the solvent. The unscreened value can be calculated by multiplying the screened value by the factor $1+\left(\varepsilon_{z, x}-1\right) A_{z, x}$, where $\varepsilon_{z, x}$ and $A_{z, x}$ are defined in Eq. (5) 
below. The Kerr constant can be either positive or negative, depending on the orientation of the ellipsoids in the electric field and their optical polarizability anisotropy.

The transient behavior in electric birefringence measurement, known as transient electric birefringence (TEB), has been used to study the rotational diffusion, size, shape, and polarization properties of objects with anisotropic geometry. The transient behavior of the birefringence reflects the alignment mechanism of these molecules under an electric field. In particular, for a suspension of elongated objects with axial symmetry and a large aspect ratio $(\geq 5)$, the rising $\left(\Delta n_{r}\right)$ and falling $\left(\Delta n_{f}\right)$ edges of the birefringence (difference between the refractive indices of the sample along and perpendicular to the applied electric field) upon application and removal of an external electric field are given, respectively, by ${ }^{12}$

$$
\Delta n_{r}(t)=\Delta n_{s}\left(1+\frac{\gamma_{z}+2 \gamma_{x}-2}{2\left(\gamma_{z}-\gamma_{x}+1\right)} \exp \left(-6 D_{R} t\right)-\frac{3 \gamma_{z}}{2\left(\gamma_{z}-\gamma_{x}+1\right)} \exp \left(-2 D_{R} t\right)\right)
$$

and $\quad \Delta n_{f}(t)=\Delta n_{s} \exp \left(-6 D_{R} t\right)$

where $\gamma_{z, x}=\frac{\mu_{z, x}^{\prime 2}}{k T\left(\alpha_{z}-\alpha_{x}\right)}, \Delta n_{s}=\Delta n(t \rightarrow \infty)$ and is given by Eq. (2), and $D_{\boldsymbol{R}}$ the rotational diffusion constant around an axis normal to the long axis of the rods. The rising edge has two exponential terms, one representing a 3-dimentional (3-D) diffusion process, which also governs the falling edge, and the other a 1-dimentional (1-D) diffusion process. The 1-D diffusion term corresponds to the orientational diffusion of the longitudinal dipole moment in the direction of the applied electric field, which results in the change in the birefringence. When the longitudinal dipole moment is absent, this term vanishes (The orientation of the transverse dipole moment does not cause the change in 
the birefringence because of the axial symmetry of the solute.), thus the rising edge of the birefringence will be symmetric to the falling edge. Benoit ${ }^{12}$ used this method to study tobacco mosaic virus (TMV), and he concluded that TMV's do not have permanent dipole moment along their long axis in aqueous solution.

\subsubsection{Experimental setup}

The setup for the electric birefringence measurement we use is a standard one ${ }^{13}{ }^{14}$. An electric field is applied transversely to a cell containing the sample between two crossed Glan-Thompson polarizers (extinction ratio $>10^{5}$ ) oriented at $45^{\circ}$ to the field. A $\lambda / 4$ plate is interposed between the sample cell and the analyzer, of which the fast axis is parallel to the polarization of the incident light. It converts the transmitted light into two lightly polarized beams. During the measurement the analyzer is rotated by $\sim 1-4^{\circ}$ from the crossed position to improve the signal to noise ratio, similar to the heterodyne technique in light scattering measurement ${ }^{15}$. This angle is much larger than the phase difference between the components of the light polarized along and perpendicular to the external field within the range of field strength applied, so that the modulation in the transmitted intensity is linear to the phase difference, and therefore to the electrically induced birefringence. The sample cell consists of two $25.0 \mathrm{~mm} \times 10.0 \mathrm{~mm}$ heavily gold-coated copper electrodes that are $\sim 1 \mathrm{~mm}$ apart in a standard $1.0 \mathrm{~cm}$ light path glass spectrometer cell, with sample solution in between. The cell is kept at $25.0 \pm 0.1{ }^{\circ} \mathrm{C}$ during the measurement. For the transient electric birefringence, the external voltage is a singlepolarity square wave applied by a pulse generator, which allows us to apply a voltage with a rise and fall time shorter than $25 \mathrm{~ns}$. A He-Ne laser $(632.8 \mathrm{~nm})$ is used as the light 
source. The transmitted light is detected with a photo-multiplier tube (PMT) with $\sim 10 \mathrm{~ns}$ time response, and the transient signal recorded with a $500 \mathrm{MHz}$ digital oscilloscope. The incident beam is attenuated in order to be in the linear range of the detector during the measurement.

\subsubsection{Calibration of the setup}

\section{a) Electrode distance}

The distance between the elctrodes is determined to be $0.12 \mathrm{~mm}$ by measuring the Kerr constant of nitrobenzene, which has a known value ${ }^{16}$ of $4.24 \times 10^{-5} \mathrm{~cm} / \mathrm{V}^{2}$ at $25^{\circ} \mathrm{C}$ for light with the wavelength of $510 \mathrm{~nm}$.

\section{b) Time response}

Special care is taken to get rid of the "ring" induced by the abrupt change in the displacement current in circuit. Besides making sure the PMT does not pick up the noise from the ground loop, magnetic shields (CONTIN, Magnetic Shielding Inc.) are applied to wrap the amplifier, PMT and all the BNC cables used. Even so the "ring" is still present, but is suppressed within $150 \mathrm{~ns}$ after the switching on of the electric field. Since it is know that the rotational diffusion of small molecules such as nitrobenzene is in picosecond scale, the time response of the whole setup was tested to be smaller than 50 ns by measuring the transient birefringence of nitrobenzene. 


\subsection{Permanent Dipole Moment of CdSe Nanorods}

The samples we measured are CdSe nanorods with various widths and lengths dispersed in cyclohexane, as shown in table I. The nanorods have excellent size monodispersity ( 5\% for width and $15 \%$ for length), as measured from transmission

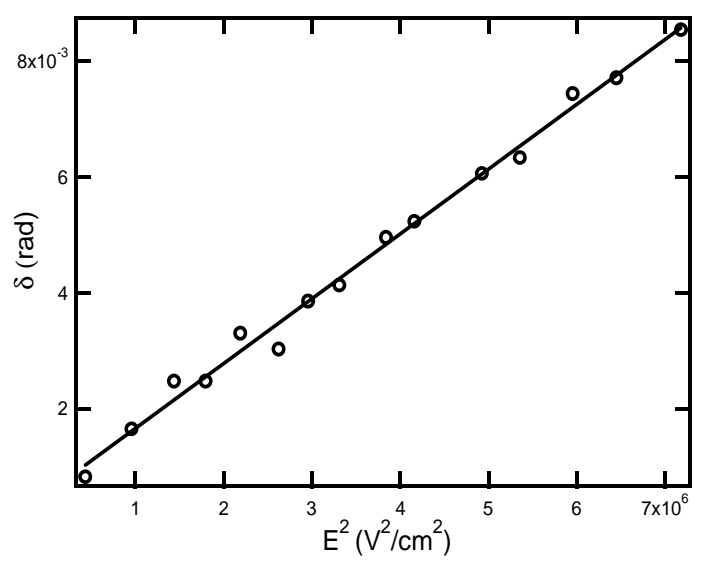

Fig. 4.1 The static electric birefringence (linear to the phase difference between the two components of the light with polarization along and perpendicular to the applied field). It is linearly proportional to the transmitted intensity under the field with various strengths when the analyzer is rotated by $\sim 4^{\circ}$ from the cross position. The sample is a dilute solution of 4.8 $\times 30 \mathrm{~nm}$ CdSe nanorods in cyclohexane.

electron micrograph images (TEM). They are coated with organic ligands such as trioctylphosphine oxide (TOPO) to make them dispersible in organic solvent. Because these surface ligands have much smaller volume and lower dielectric constants comparing with the nanocrystals, their contribution to the electric birefringence is negligible. This is proven by the blank experiments, which show that the birefringence caused by the surface ligands is at least three orders of magnitude smaller. The solutions of CdSe nanorods we measured were maintained dilute (number density $<<1 /$ length ${ }^{3}$ ) to avoid interrod coupling. The charging of the CdSe nanocrystals is not significant because 
no accumulation of nanorods at either of the electrodes was observed when a static voltage was applied for hours.

Table I

\begin{tabular}{|c|c|c|c|c|c|c|c|c|c|}
\hline $\begin{array}{l}\text { Width } \\
(\mathrm{nm})^{\mathrm{a}}\end{array}$ & $\begin{array}{l}\text { Length } \\
(\mathrm{nm})^{\mathrm{a}}\end{array}$ & $\begin{array}{l}\text { Length } \\
(\mathrm{nm})^{\mathrm{b}}\end{array}$ & $\begin{array}{l}\text { Volume } \\
\left(\mathrm{nm}^{3}\right)\end{array}$ & $\begin{array}{l}\left(6 D_{R}\right)^{-1} \\
(\mu \mathrm{s})\end{array}$ & $\frac{3 \gamma}{\gamma-2}$ & $\gamma$ & $\begin{array}{c}\frac{\alpha_{\|}}{4 \pi \varepsilon_{0}} \\
\left(\mathrm{~nm}^{3}\right)\end{array}$ & $\begin{array}{l}\frac{\alpha_{\perp}}{4 \pi \varepsilon_{0}} \\
\left(\mathrm{~nm}^{3}\right)\end{array}$ & $\begin{array}{l}\mu_{z} \\
\text { (Debye) }\end{array}$ \\
\hline $\begin{array}{l}3.1 \\
\pm 0.1\end{array}$ & $\begin{array}{l}58 \\
\pm 6\end{array}$ & $\begin{array}{l}60 \\
\pm 0.3\end{array}$ & $\begin{array}{l}302 \\
\pm 24\end{array}$ & $\begin{array}{l}3.61 \\
\pm 0.05\end{array}$ & $\begin{array}{l}5.43 \\
\pm 0.38\end{array}$ & $\begin{array}{l}4.47 \\
\pm 0.38\end{array}$ & $\begin{array}{l}94.4 \\
\pm 12.5\end{array}$ & $\begin{array}{l}31.1 \\
\pm 4.1\end{array}$ & $\begin{array}{l}153.4 \\
\pm 23.9\end{array}$ \\
\hline $\begin{array}{l}4.8 \\
\pm 0.2 \\
\end{array}$ & $\begin{array}{l}26 \\
\pm 4\end{array}$ & $\begin{array}{l}30 \\
\pm 0.5\end{array}$ & $\begin{array}{l}361 \\
\pm 29 \\
\end{array}$ & $\begin{array}{l}0.81 \\
\pm 0.04\end{array}$ & $\begin{array}{l}4.24 \\
\pm 0.12 \\
\end{array}$ & $\begin{array}{l}6.84 \\
\pm 0.46 \\
\end{array}$ & $\begin{array}{l}99.9 \\
\pm 14.0\end{array}$ & $\begin{array}{l}38.1 \\
\pm 5.3 \\
\end{array}$ & $\begin{array}{l}209.9 \\
\pm 32.6\end{array}$ \\
\hline $\begin{array}{l}3.8 \\
\pm 0.2\end{array}$ & $\begin{array}{l}20 \\
\pm 3\end{array}$ & $\begin{array}{l}23 \\
\pm 1.6 \\
\end{array}$ & $\begin{array}{l}174 \\
\pm 18\end{array}$ & $\begin{array}{l}0.39 \\
\pm 0.08\end{array}$ & $\begin{array}{l}4.82 \\
\pm 0.87\end{array}$ & $\begin{array}{l}5.30 \\
\pm 1.15\end{array}$ & $\begin{array}{l}47.7 \\
\pm 8.2\end{array}$ & $\begin{array}{r}18.3 \\
\pm 3.1 \\
\end{array}$ & $\begin{array}{l}126.4 \\
\pm 36.9\end{array}$ \\
\hline $\begin{array}{l}3.0 \\
\pm 0.1\end{array}$ & $\begin{array}{l}51 \\
\pm 6\end{array}$ & $\begin{array}{l}54 \\
\pm 0.6\end{array}$ & $\begin{array}{l}254 \\
\pm 20\end{array}$ & $\begin{array}{l}2.83 \\
\pm 0.10\end{array}$ & $\begin{array}{l}6.77 \\
\pm 0.82\end{array}$ & $\begin{array}{l}3.59 \\
\pm 0.34\end{array}$ & $\begin{array}{l}79.2 \\
\pm 11.5\end{array}$ & $\begin{array}{l}26.2 \\
\pm 3.8\end{array}$ & $\begin{array}{l}126.3 \\
\pm 21.4\end{array}$ \\
\hline $\begin{array}{l}3.0 \\
\pm 0.1\end{array}$ & $\begin{array}{l}33 \\
\pm 4\end{array}$ & $\begin{array}{l}35 \\
\pm 1.1\end{array}$ & $\begin{array}{l}165 \\
\pm 14\end{array}$ & $\begin{array}{l}0.93 \\
\pm 0.09\end{array}$ & $\begin{array}{l}8.17 \\
\pm 2.81\end{array}$ & $\begin{array}{l}3.16 \\
\pm 0.64\end{array}$ & $\begin{array}{l}49.8 \\
\pm 8.2\end{array}$ & $\begin{array}{l}17.1 \\
\pm 2.8\end{array}$ & $\begin{array}{l}95.7 \\
\pm 24.6\end{array}$ \\
\hline
\end{tabular}

a) Measured from TEM images.

b) Calculated with Eq. (4) from $D_{R}$ obtained from the electric birefringence measurement. $1.1 \mathrm{~nm}$ is subtracted as the capping ligands, e. g. TOPO.

Figure 4.1 shows the static birefringence of transmitted light $v s$ electric field. Within the range of the field strength we applied, only linear behavior is observed. These nanorods under electric field have birefringence of the same sign as nitrobenzene, indicating that they align parallel to the electric field ${ }^{17}$. Figure 4.2 (a) shows a typical transient electric birefringence curve measured for CdSe nanorods. As suggested by Eq. (3), the asymmetric falling and rising edges indicate the existence of a permanent electric dipole moment along the long axis. The hexagonal symmetry of CdSe nanorods allows us to approximately treat them as being axially symmetric, and therefore the transverse permanent dipole moment $\left(\mu_{x}^{\prime}\right)$ is zero. We fit the TEB curves (e.g. Fig. 2 (b) and (c)) 
with equations (3) to obtain the rotational diffusion constants and the ratio of longitudinal permanent dipole moment to the polarizability anisotropy $(\gamma)$, as listed in table I.
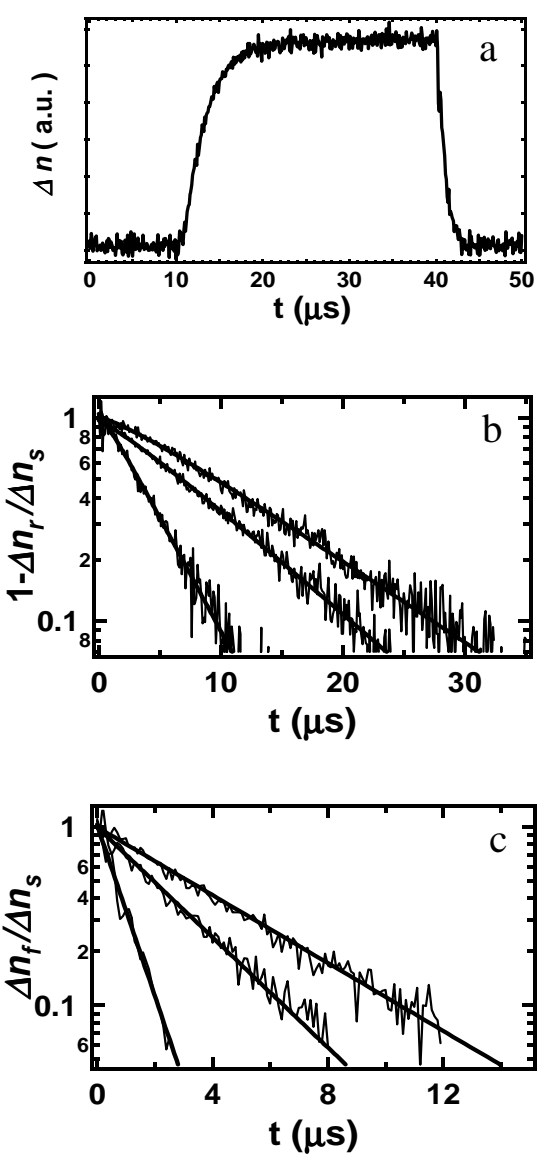

Fig. 4.2 (a) A typical TEB curve (in linear scale) upon the application and subsequent removal of the electric field. The asymmetric rising and falling edges indicate the existence of a permanent dipole moment along the long axis of the nanorods. In this particular case the CdSe nanorods are $4.8 \mathrm{~nm}$ wide and $30 \mathrm{~nm}$ long. (b) and (c) The logarithm scale plots of the fit for the rising and falling edges in the form of equations (1) for the TEB curves of three CdSe nanorod samples. $\Delta \mathrm{n}_{\mathrm{s}}$ is the saturation value of $\Delta \mathrm{n}(\mathrm{t})$. In both (b) and (c) from left to right the CdSe nanorods are $3.0 \times 35,3.0 \times 54,3.1 \times 60$ $\mathrm{nm}$ (in width and length) respectively.

From the rotational diffusion constants $D_{R}$ obtained by fitting the TEB curves, we can get the length of the nanorods $l$ by the equation ${ }^{18}$

$$
D_{R}=\frac{3 k_{b} T}{\pi \eta l^{3}}\left(\ln \frac{2 l}{d}-0.8\right)
$$

where $d$ is the width of the nanorods, as determined from TEM images, and $\eta$ the viscosity of the solution $\left(0.98 \mathrm{mPa} \cdot \mathrm{s}\right.$ for cyclohexane at $\left.25^{\circ} \mathrm{C}\right)$. The calculated lengths 
agree very well (within 10\%) with the results from TEM images, which confirms that the TEB signals we obtained are due to the CdSe nanorods. Because $D_{R}$ shown in equation (4) is very sensitive to the length but not to the width, we can use the TEB method to measure the length of the nanorods. This method has the advantage that the result is an ensemble average and therefore more representative, in contrast to the local sampling of the TEM method.

In order to get the permanent dipole moment from $\gamma$, we calculate the electric polarizability of CdSe nanorods by assuming a revolute prolate shape, so that the principle axes of polarizability coincide with the geometrical axes. The polarizabilities shown in table I are calculated by ${ }^{19}$

$$
\alpha_{z, x}=\varepsilon_{0} v\left(\varepsilon_{z, x}-1\right) /\left[1+A_{z, x}\left(\varepsilon_{z, x}-1\right)\right]
$$

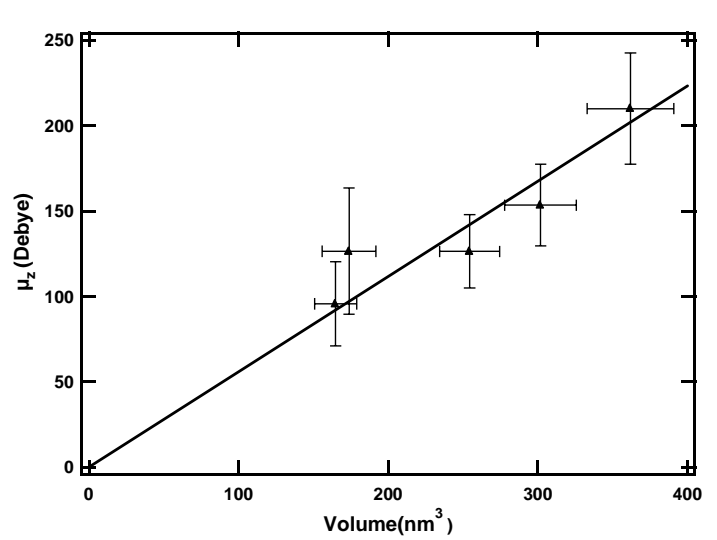

Fig. 4.3 The unscreened dipole moment of CdSe nanocrystals. The solid triangles are the values measured with the TEB method (also listed in Table I), and the straight line is the best fit, with a slope of $0.19 \mu \mathrm{C} / \mathrm{cm}^{2}$. where $v$ is the volume of individual nanorod, $\varepsilon$ 's the relative dielectric constants between nanorods and the solvent along $(z)$ or perpendicular $(x)$ to the long axis of the nanorods, and A's the geometrical factors that can be calculated from the dimension of the nanorods. The dielectric constants of CdSe nanorods are taken as bulk material values $\left(\varepsilon_{/ /}=10.2, \varepsilon_{\perp}=9.33\right)$, and that of cyclohexane is taken as 2.02 .

The unscreened permanent dipole 
moments $\mu_{\mathrm{z}}$ of five nanorod samples are listed in table I, and plotted in figure 4.3 vs. their volume. Considering the ensemble nature of the measurement, these are the root mean square dipole moment of the samples. It is an average not only over the finite size distribution, but also over the possible structural distribution. Within the experimental error, however, a linear dependence of $\mu_{z}$ vs. volume is obtained, and no correlation between rod length or width and $\mu_{z}$ is observed. This is consistent with theoretical analyses that the polarity is intrinsic to the crystallographic lattice of CdSe due to the lack of inversion symmetry. Our results are not consistent with a random dipole moment resulting from trapped surface charges, as proposed in the case of spherical CdSe nanocrystals, which have much smaller volume. From the slope we get the polarization of CdSe nanorods to be $0.19 \mu \mathrm{C} / \mathrm{cm}^{2}$ along the $c$-crystallographic axis. This is in reasonably good agreement with the value of $0.6 \mu \mathrm{C} / \mathrm{cm}^{2}$, as estimated ${ }^{5}$ from a phenomenological rule that was only proven experimentally for ferroelectric materials.

It is well known that migrating charges may contribute to the electric dipole moment measured by the TEB method ${ }^{20}$. Like the fluctuating protons on the surface of proteins in aqueous solution, the hopping of trapped charges between different trapping sites on the CdSe nanocrystal surfaces would result in a non-vanishing mean square average dipole moment even with a vanishing mean dipole moment. This effect can be modeled with a time-dependent electric polarizability. It has been shown that if the induced dipole due to the migrating charges is predominant over that caused by the lattice, and we assume a single exponential form for the polarizability, i. e. $\alpha_{/ /}-\alpha_{\perp}=q_{l}+q_{c} \cdot(1-\exp (-t / \tau))$, where $q_{l}$ and $q_{c}$ are the anisotropy of the 
polarizability contributed by the lattice and the migrating surface charges respectively, the rising edge of the TEB curve would be expressed as

$$
\Delta n_{r}(t)=\Delta n_{s}\left(1-\exp \left(-6 D_{R} t\right)-\exp (-t / \tau)+\exp \left\{-\left(1 / \tau+6 D_{R}\right) t\right\}\right),
$$

where $\tau$ is related to the diffusion constant of the surface charges on the surface and scales linearly with the surface area of the nanorods, and for long rods, linearly to the length. This is not consistent with our experimental results, which indicates that the contribution of the migrating charges on the surfaces of the nanorods to the permanent dipole moment is not significant.

It has been proposed that the surface localized charges result in the non-zero dipole moment in CdSe and $\mathrm{Zn}$ Se spherical nanocrystals ${ }^{6}$. However, the longitudinal permanent dipole moment for the CdSe nanorods have comparable magnitudes with those reported for spherical nanocrystals with much smaller volume, indicating that the localized charges do not contribute significantly for nanorods. For spherical particles it was assumed $^{6}$ that there are only one pair of localized charges on the surfaces regardless of the particle size, and it explained the experimental results that the dipole moment scales linearly with the diameter of the particles. For nanorods, however, this assumption contradicts our experimental results. For example, if the localized charges generated a transverse dipole moment, there would have been an inversion in the signs of the electric birefringence with increasing volume. Therefore there have to be multiple pairs of localized charges if there are any, and their contributions to the dipole moment cancel out. 
The large permanent electric dipole moment along the long axes of the nanorods has important consequences on the linear and nonlinear optical properties of CdSe nanorods. The electric field generated by the electric dipole will change the distribution of the photo-excited carries, and therefore the energy level structures, fluorescence lifetime, and even the selection rules. Resonance Raman depolarization ${ }^{21}$, two-photon excited fluorescence spectroscopy ${ }^{2}$ and Hyper-Rayleigh scattering ${ }^{22}$ experiments performed on spherical CdSe nanocrystals can be easily extended to the nanorods. Furthermore, the longitudinal dipole moment may affect the orientational order of CdSe nanorods. For example, according to a numerical simulation performed on the lyotropic liquid crystalline phase formation of rigid rods, adding a central electric dipole moment shifts the isotropic-nematic phase transition to higher concentration ${ }^{23}$ and may also stabilize a smectic phase with respect to the nematic phase, while a study on elongated ellipsoids with off-centered longitudinal dipoles ${ }^{24}$ showed the formation of modulated antiferroelectric domains. 


\section{Appendix}

\section{Experimental details of the TEB measurement}

The schematic of the TEB setup is shown below, where the Glan-Thompson polarizers have extinction ratio of $\sim 10^{5}$, and a $500 \mathrm{MHz}$ oscilloscope is used.

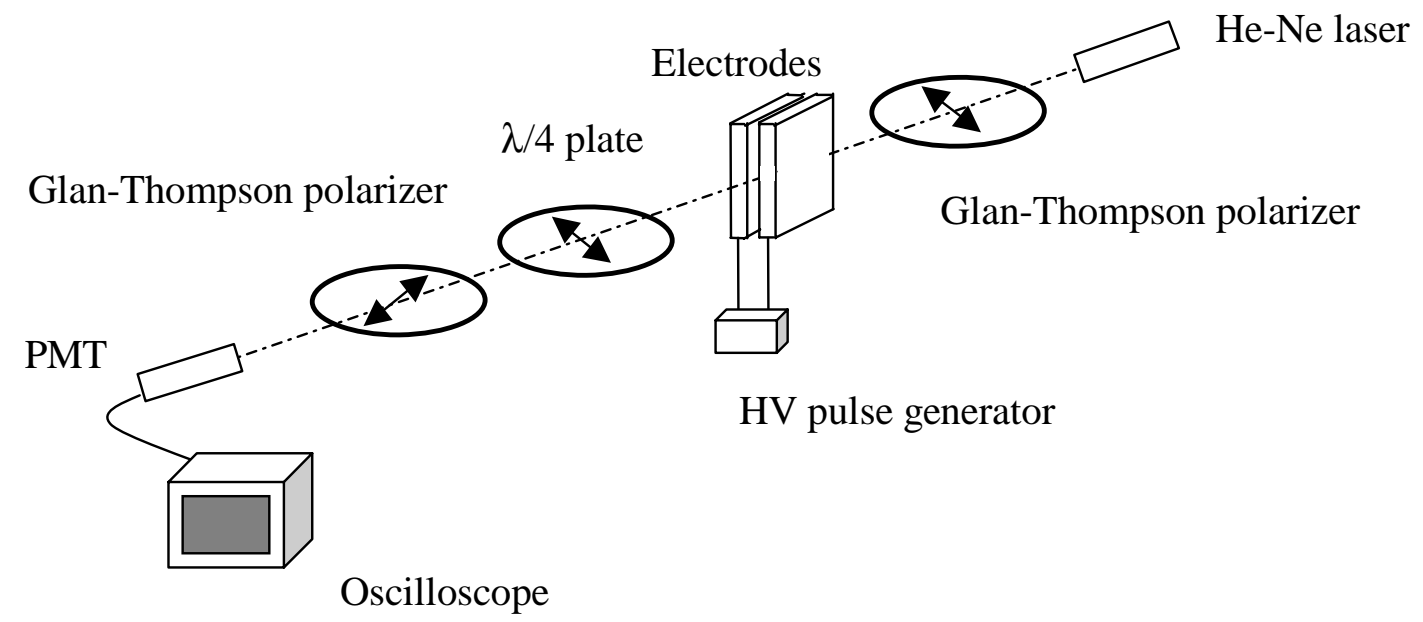

The configuration of the directions of the polarizers and applied electric field is shown in the next page. After the incident beam goes through the sample, the fields parallel and perpendicular to the applied electric field $\boldsymbol{E}$ will be:

$$
E_{\|}=\frac{E_{0}}{\sqrt{2}} \exp \left[i\left(k_{\|} d+k_{0} z-\omega t\right)\right], \quad E_{\perp}=\frac{E_{0}}{\sqrt{2}} \exp \left[i\left(k_{\perp} d+k_{0} z-\omega t\right)\right],
$$

where $E_{0}$ is the amplitude of the linearly polarized incident beam, which is along the $y$ direction, and $k_{0}, k_{\|}$and $k_{\perp}$ equal to $2 \pi n_{0} / \lambda, 2 \pi n_{\|} / \lambda$ and $2 \pi_{n_{\perp}} / \lambda$, respectively. Therefore,

$$
E_{x}=-\frac{E_{0}}{\sqrt{2}} \exp \left(i k_{\perp} d\right)[\exp (i \delta)-1], \quad E_{y}=\frac{E_{0}}{\sqrt{2}} \exp \left(i k_{\perp} d\right)[\exp (i \delta)+1]
$$


where the common factor $\exp \left[i\left(k_{0} z-\omega t\right)\right]$ is omitted and

$$
\delta=\left(k_{\|}-k_{\perp}\right) d=2 \pi \Delta n / \lambda .
$$

After the quarter-wave plate,

$$
E_{x}=E_{0} \exp \left[i\left(\delta / 2+k_{\perp} d\right)\right] \sin (\delta / 2), \quad E_{y}=E_{0} \exp \left[i\left(\delta / 2+k_{\perp} d\right)\right] \cos (\delta / 2)
$$

Then the intensity transmitted through the analyzer is

$$
I=I_{0} \sin ^{2} \alpha-I_{0} \sin (2 \alpha-\delta / 2) \sin (\delta / 2),
$$

where $I_{0}=E_{0}^{2}$. When $\alpha$

$>\delta / 2, \Delta I=I-I_{0} \sin ^{2} \alpha \propto \delta$,

while when $\alpha=0, \Delta \mathrm{I} \propto \delta^{2}$,

i.e. for small $\delta$, having a

non-zero $\alpha$ will greatly

increase the sensitivity of

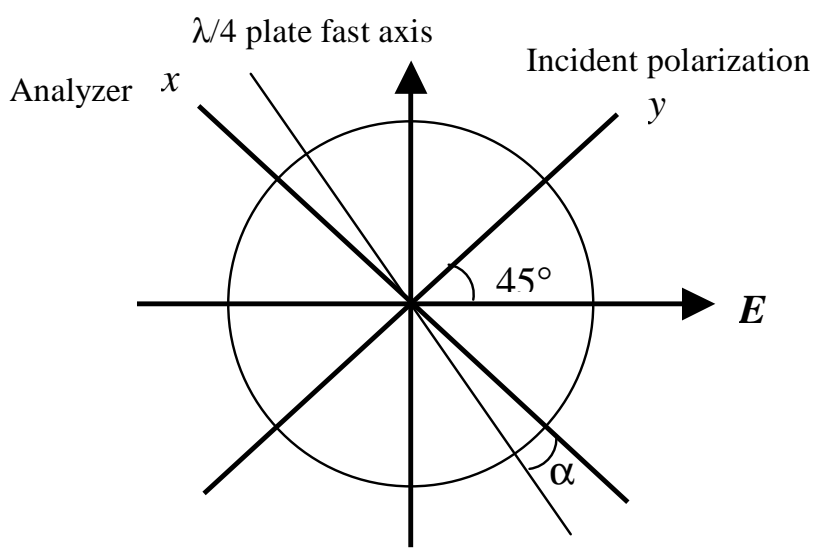

the measurement.

In order to measure the absolute values of $\delta$ and the Kerr constant, it is necessary to measure $I_{0}$. This is done by measure the transmitted intensity with different $\alpha$, while the applied electric field is off. Then the Kerr constant is measured as the slope of the $I \sim E$ curve with a fixed $\alpha$ at the low field limit. The distance between the two electrodes is calibrated by measuring the Kerr constants of some liquids and comparing them with literature values, such as nitrobenzene. 


\section{Reference and Notes}

${ }^{1}$ L.-S. Li and A. P. Alivisatos, Phys. Rev. Lett. 2003, 90 (9), 097402.

${ }^{2}$ Schmidt, M. E.; Blanton, S. A.; Hines, M. A.; Guyot-Sionnest, P. J. Chem. Phys. 1997, $106(12), 5254-5259$.

${ }^{3}$ Rabani, E.; Hetényi, B.; Berne, B. J.; Brus, L. E. J. Chem. Phys. 1999, 110 (11), 53555369.

${ }^{4}$ Huong, N. Q.; Birman, J. L. J. Chem. Phys. 1998, 108 (5), 1769-1772.

${ }^{5}$ Blanton, S. A.; Leheny, R. L.; Hines, M. A.; Guyot-Sionnest, P. Phys. Rev. Lett. 1997, $79(5), 865-868$.

${ }^{6}$ Shim, M.; Guyot-Sionnest, P. J. Chem. Phys. 1999, 111 (15), 6955-6964.

${ }^{7}$ O'Konski, C. T. in Encyclopedia of Polymer Science and Technology, Interscience: New York, 1968. Vol. 9, 551-590.

8 Yoshioka, K.; Watanabe, H. in Physical Principles and techniques of Protein Chemistry, Ed. S. J. Leach, Academic Press: New York, 1969. Pt. A, 335-367.

${ }^{9}$ Böttcher, C. J. F. Theory of Electric Polarization, Elsevier Scientific: New York, 1973.

${ }^{10}$ O’ Konski, C. T.; Yoshioka, K.; Orttung, W. H. J. Phys. Chem. 1959, 63 (10), 15581565.

${ }^{11}$ I. Tinoco, J. Am. Chem. Soc., 1955, 77 (17), 4486-4489.

${ }^{12}$ H. Benoit, Ann. Phys. (Paris) 1951, 6, 561-609.

${ }^{13}$ C. T. O'Konski and B. H. Zimm, Science 1950, 111 (2875), 113-116.

${ }^{14}$ J. Newman and H. L. Swinney, Biopolymers 1976, 15, 301-315.

${ }^{15}$ Bernes, B. J.; Pecora, R. Dynamic Light Scattering, Dover Pub. Inc.: New York 2000, 6. 
${ }^{16}$ I. Tinoco, J. Am. Chem. Soc. 1957, 79 (16), 4336-4338.

17 The sign of the electric birefringence, or of the Kerr constant is determined by two factors: anisotropy of the polarizability at the optical frequency (632.8nm for our case), and the degree of the orientation that is determined by the permanent dipole moment and the anisotropy of the static polarizability. The former factor, as calculated with Eq. (2) but with $n_{/ /, \perp}^{2}$ replacing the $\mathcal{\varepsilon} \mathrm{s}$, is positive, therefore we can determine the degree of the orientation to be positive, i. e. the rods align along the electric field.

${ }^{18}$ J. M. Burgers, Verhandel. Koninkl. Ned. Akad. Wetenschap. Afdeel. Natuurk., Sec. 1, 1938, $16(4), 113$.

${ }^{19}$ J. A. Osborn, Phys. Rev. 1945, 67(11-12), 351-357.

${ }^{20}$ J. G. Kirkwood and J. B. Shumaker, Proc. Natl. Acad. Sci. 1952, 38, 855 -862.

${ }^{21}$ J. J. Shiang, A. V. Kadavanich, R. K. Grubbs and A. P. Alivisatos, J. Phys. Chem. 1995, $99(48), 17417-17422$.

${ }^{22}$ M. Jacobsohn and U. Banin, J. Phys. Chem. B 2000, 104 (1), 1-5.

${ }^{23}$ S. C. McGrother, A. Gil-Villegas and G. Jackson, Mol. Phys. 1998, 95 (3), 657-673.

${ }^{24}$ R.Berardi, S. Orlandi and C. Zannoni, Chem. Phys. Lett. 1996, 261 (3), 357-360. 


\section{Chapter 5 Assembly of Nanorods on a Substrate}

\subsection{Motivation}

Despite the fact that nanocrystals are often referred as "artificial atoms", the interactions between them are very different from those between atoms and small molecules. Even though the strong chemical bonds between nanocrystals such as ionic bonds and covalent bonds are not common, the van der Waals interactions between them are much stronger due to their sizes and high polarizabilities ${ }^{1}$. In order to study the interaction between nanocrystals, the formation and properties of the crystals of nanocrystals, or "supercrystals" and "superlattices", have been studied ${ }^{23}$.

On the other hand, in order to apply the anisotropic optical and electrical properties of CdSe nanorods in devices, such as polarizing light emitting diode and solar cells, we need to align them, preferentially on a substrate, on a large scale. This is also desirable for the study of the anisotropic ensemble properties of the nanorods such as optical and x-ray absorption, surface-selectivity of the ligands, etc. For spherical nanocrystals there have been various ways to assemble them into large scale superlattices, such as supercrystal growth by the slow solvent evaporation method or diffusing a poor solvent into a nanocrystal solution ${ }^{2}$, Langmuir film techniques ${ }^{4}$, etc. However, the geometric anisotropy of the nanorods makes it very difficult to achieve their order on a comparable scale with the same methods. For example, both methods mentioned above have been applied to $\mathrm{Au}^{6}$ or $\mathrm{BaCrO}_{4}$ nanorods ${ }^{7}$, but the area of alignment spans only a few hundred 
nanometers. Recently a synthesis-coupled assembly method was invented ${ }^{8}$, but the aligned superstructures are also far from being macroscopic.

In this chapter the approaches we took in the early stage of our attempt to align the CdSe nanorods discussed, which include the application of oriented substrates, and the spontaneous crystallization by slow solvent evaporation method. In neither cases macroscopic alignment is achieved. However the results reveal the possible mechanisms of the alignment and the formation of these superstructures, and inspire us to come up with new approaches, which are described in the following chapters. 


\subsection{Alignment of CdSe Nanorods on an Aligned Substrate}

One approach to large areas of alignment is to break the isotropic symmetry of the environment, such as the applications of pretreated substrates and shear flows. This idea has been widely used in liquid crystal display industry ${ }^{9}$, such as alignment of small molecular thermotropic liquid crystals on rubbed polyimide film. This has been attributed to a mechanism similar to heteroepitaxy growth $^{10}$, even though pure geometric mechanism ${ }^{11}$ has also been proposed. On the other hand, shear flow in microchannels has been used to align nanowires, but only on the scale of a few nanowires. In this section we use an oriented substrate to align CdSe nanorods, as an analog of the pretreated surface method in the liquid crystal display industry. The substrate we used is a frictiontransferred Teflon film ${ }^{12}$. This method of substrate treatment is particularly simple and the aligning effects on small molecular thermotropic liquid crystals have been proven to be as successful as the traditional polyimide substrate.

To prepare the aligned Teflon film, a Teflon bar is rubbed over a regular microscope glass slide. A thin film is left on the slide due to the friction. For characterization purposes, the film is floated off by dipping the glass slide into water. The film is then picked up with a blank 400 mesh copper TEM grid (Ted Pella). Under TEM, aligned grooves with thickness around 50-100 $\mathrm{nm}$ are observed, which are also reported in literatures. Electron diffraction is performed on the film to verify the orientational order of the film, and the diffraction pattern indicates the preferential alignment of the polymer backbones, as shown in figure 5.1, which is attributed to the stretching of the polymer backbone against the glass substrate and the subsequent crystallization. 

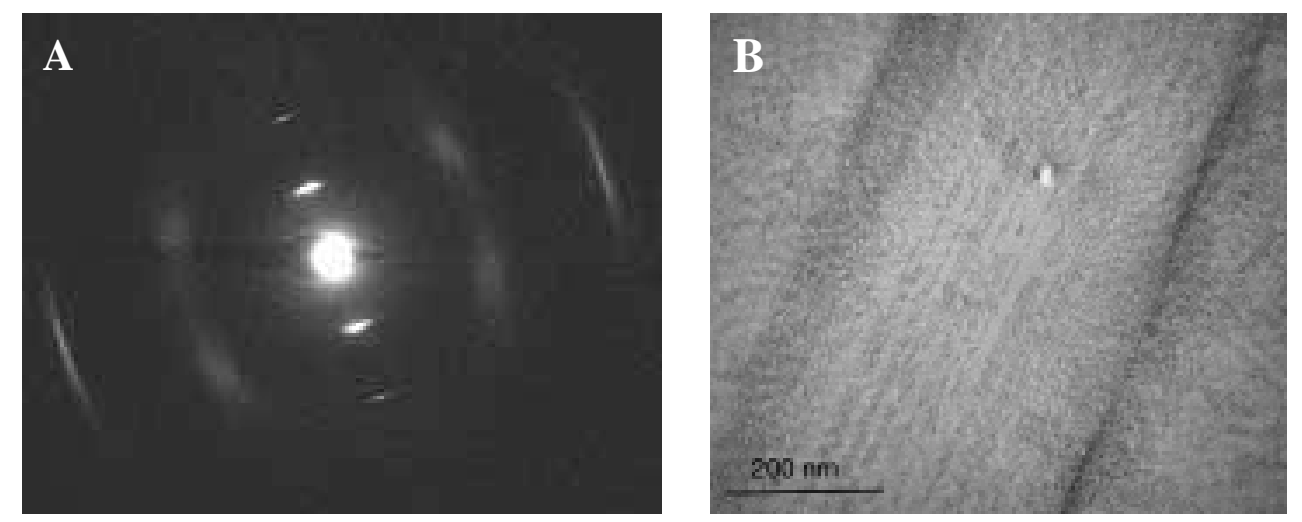

Fig. 5.1 A) Electron diffraction pattern of the orientated Teflon film, indicating the uniaxial orientation of the crystalline domains. B) Aligned area of CdSe nanorods on the orientated Teflon film.

Dilute solution of CdSe nanorods are dropped onto the freshly prepared Teflon film on a glass slide. After the solvent is evaporated, the film is floated off in water and picked up with a copper grid. Under TEM, areas of aligned nanorods are observed, even though the nanorods are not closely packed (figure 5.1 (B)). This indicates that the interactions between the nanorods themselves plays little role, and therefore the interaction between the nanorods and the substrate dominates. On the other hand, this also suggests the alignment mechanism is different from that proposed for the small molecule thermotropic liquid crystals. Because of the large size of the nanocrystals compared with the polymer chains and the fact that the nanocrystals are coated with organic molecules, the heteroepitaxy mechanism should not be important. Instead, this may be due to the confinement of the nanorods in the grooves on the surface of the film and their freedom to rotate in the presence of the solvent. 


\subsection{Superlattices of CdSe Nanorods by Slow Solvent Evaporation ${ }^{13}$}

The other approach to achieving alignment of nanorods is taking advantage of spontaneous symmetry breaking when a solution of nanocrystals is completely dried. This is the mechanism governing various crystallization processes. This method has been applied on Au nanorods ${ }^{6}$, but because this process is very sensitive to the environment, such as temperature, material transport etc., large-scale superlattice hasn't been achieved.

In order to obtain structures thin enough for TEM study, a freshly cleaved mica sheet coated with a thin carbon film is immersed vertically into a dilute solution of $\mathrm{CdSe}$ nanorods in cyclohexane. As the solvent evaporates, the liquid level sweeps over the substrate, depositing CdSe nanorods behind when the solvent is completely gone. The convective flow caused by the solvent evaporation carries the nanorods from the bulk solution to the liquid-solid-air interface and can make the solution locally very

concentrated, and therefore phenomena such as nanorod crystallization can occur ${ }^{14}$. The carbon film is then floated off on water and picked up on a TEM grid. We also used commercial carbon coated copper grids and obtained the same result.

Because of the inhomogeneity in local concentration and the nonequilibrium nature of the solvent evaporation method, assemblies with different levels of order were left on the carbon film, ranging from amorphous to crystalline. Figure 5.2 shows the typical structures observed in ordered regions formed by $3.7 \mathrm{~nm} \times 18 \mathrm{~nm}$ CdSe nanorods in different areas on the same TEM grid. Figure 5.2(A) shows an area in which the nanorods are positioned randomly but oriented overall in the same direction. Figures 5.2(B) and 
5.2(C) show two different types of crystalline structures. In 5.2(B) a multi-layered structure is shown, with CdSe nanorods connecting to one another head-to-tail to form linear arrays. Different layers appear to be different domains, and they have a $\sim 60^{\circ}$ angle with respect to each other. Due to the overlap between different layers and the finite length distribution of these nanorods, no overall periodicity is observed along the
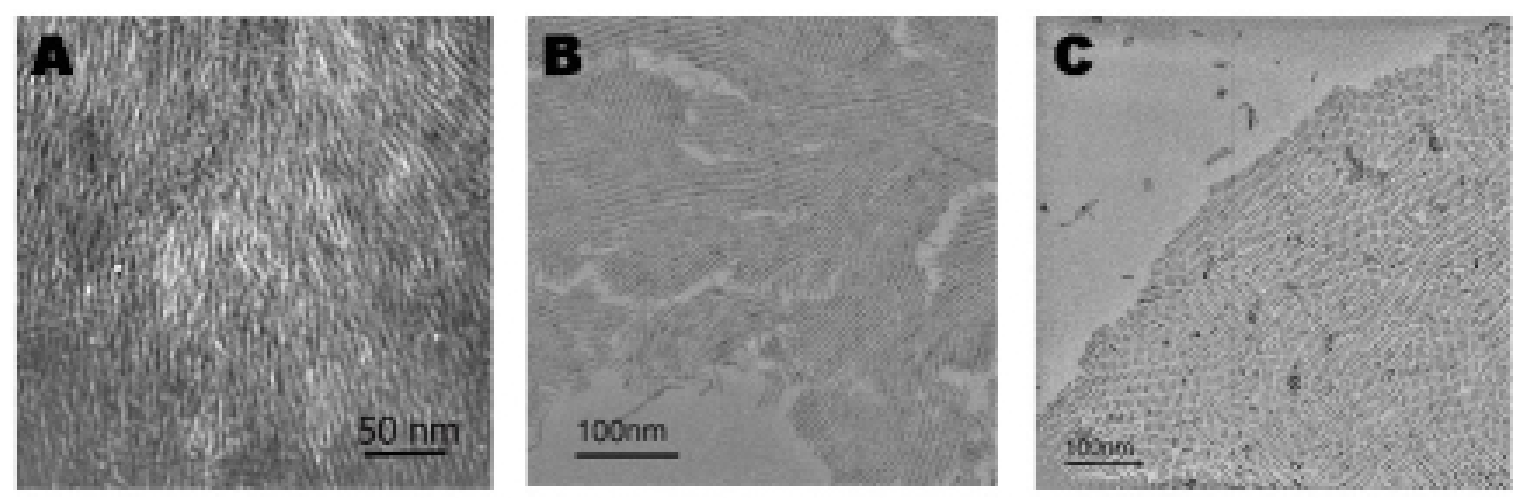

Fig. 5. 2. Transmission electron micrograph images of typical superlattice structures formed when CdSe nanorods are deposited on a carbon film from a solution by slow solvent evaporation method. All the nanorods are $3.7 \mathrm{~nm}$ in diameter and $18 \mathrm{~nm}$ in length. A) Structures in which the nanorods have an overall orientational order but random positions. B) Multi-domain linear arrays. Within each domain, the nanorods have lateral positional order but no longitudinal order. Different domains have a $\sim 60^{\circ}$ angle in orientation. C) Monolayer in which the rods have positional order in both lateral and longitudinal directions.

direction of the line structure. Within each domain periodicity along the direction perpendicular to the line directions can be seen, with an interline spacing of $\sim 1.1 \mathrm{~nm}$, which corresponds to twice the length of the capping molecules on the nanorods. In each line the nanorods are closely packed. This structure is particularly interesting, because in each domain, the lines have a high degree of positional order, and by replacing the 
surfactants on the surface of these nanorods with other molecules, we may be able to control the distance between these lines.

In the linear array structures, very frequently vortex structures are observed, as shown in figure 5.3 (A-C). In particular, figure 5.3(C) demonstrates an isolated vortex structure, together with its small angle electron diffraction pattern, suggesting it being formed spontaneously on the amorphous substrate. The change of the orientations of nanorods around the vortex clearly resembles a disclination of strength +1 in a nematic liquid
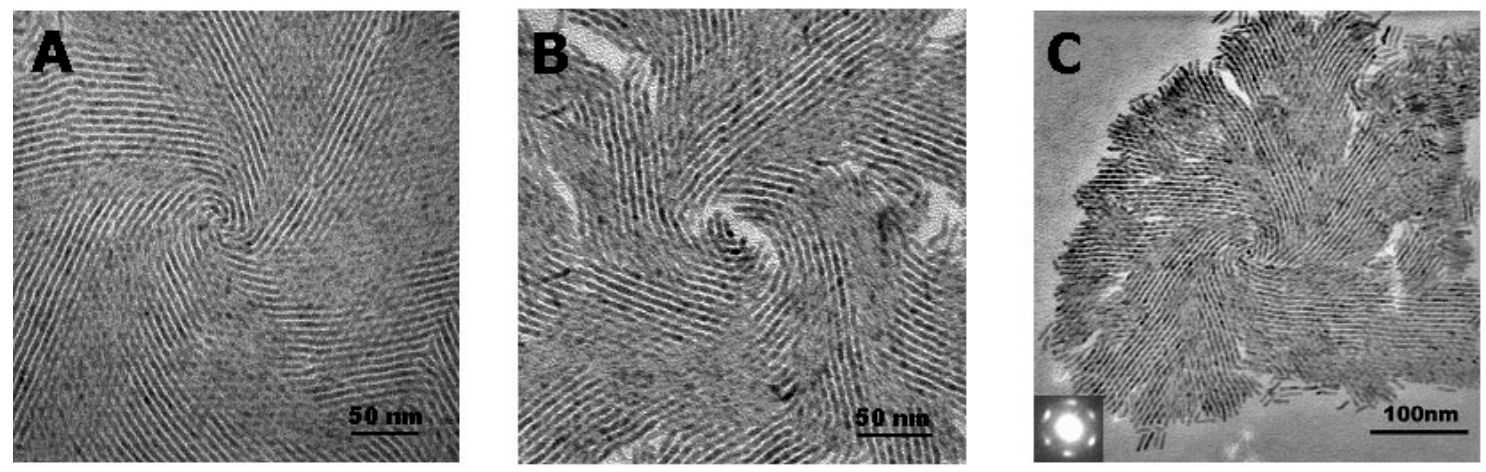

Fig.5.3. Vortex structures in the linear arrays. In all of the vortex structures, six branches can be identified. It is believed that the linear arrays nucleate at these vortices, and each branch of them evolves into a domain. Figure 3C) shows an isolated vortex, suggesting the spontaneous formation of these structures. The inset is the small angle electron diffraction pattern of the selected area. It clearly shows the six-fold symmetry of the packing.

crystalline phase ${ }^{15}$. Recently, similar disclination structures have also been observed in $\mathrm{BaCrO}_{4}$ nanorod assemblies with $\mathrm{TEM}^{7}$, where the high compression in the Langmuir film prevents the distortion from further relaxation. In our case, the CdSe nanorods around the cores of these vortex structures are divided into six branches, with $\sim 60^{\circ}$ angle between each other. 
The long-range angular correlation between the crystalline domains makes us believe that the vortex structures shown in figure 5.3 are the nucleation sites of the crystallization. Figure 5.4 show TEM images of large areas of multi-domained linear arrays with their Fourier transformation patterns, in which six-fold symmetry can be clearly seen. We attribute this to the propagation of the six-fold pattern around the nucleation sites via the growth of the superlattices. The large area of the six-fold symmetry suggests that either the whole area evolve from a single nucleation site or the nucleation events are correlated. The six-fold orientational correlation decays with increasing area, as indicated by the increasing diffuseness in the electron diffraction patterns (from 5.4 B to $5.4 \mathrm{~A}$ ), due to the bending along the linear arrays.

Since these structures are formed on amorphous carbon films, the six-fold
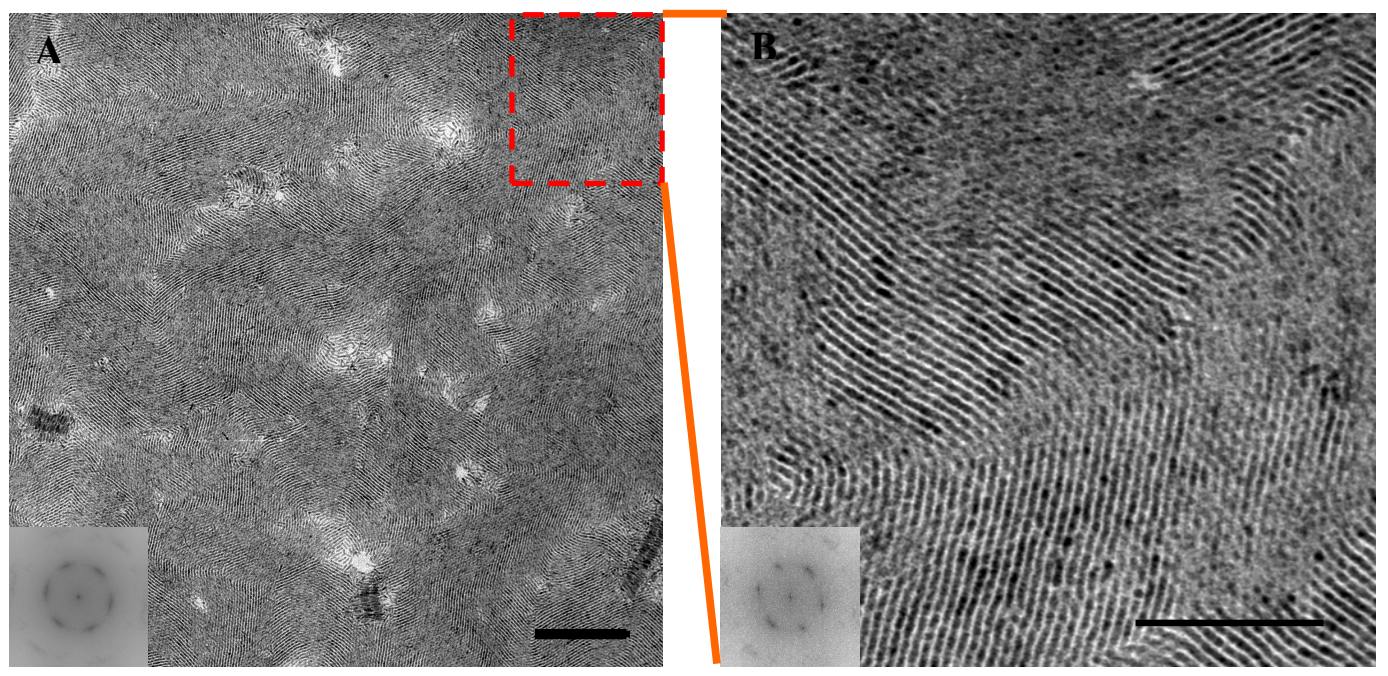

Fig. 5.4 Extended areas of the superlattices of CdSe nanorods observed with TEM. The scalebar is 500 $\mathrm{nm}$ for the left, and $100 \mathrm{~nm}$ for the right hand side respectively. The insets are the Fourier transform of the images. A $60^{\circ}$ angle between different super crystalline domains is very common in the superlattice of CdSe nanorods. Fourier transform shows that the angular correlation exists over a length scale up to micron. The image on the right side is $1 / 16$ of the one on the left side. 
orientational correlation is unlikely due to substrate effects, but should be due to the inter-particle interactions. Formation of linear arrays from dried lyotropic nematic solution of tobacco mosaic virus (TMV) (figure 5.5 (A)) has been observed with a combination of polarizing optical microscopy and atomic force microscopy (AFM) ${ }^{16}$. TMV's are much larger ( $300 \mathrm{~nm}$ in length and $18 \mathrm{~nm}$ in width) compared with CdSe nanorods, but they also have a rodlike shape. They have been well studied for the formation of lyotropic nematic phase (for more details see Chapter 6) when they are dispersed in water. Interestingly, in the linear arrays of tobacco mosaic viruses, a critical bending angle of $60^{\circ}$ was also observed, which is accompanied by the breaking of individual virus particles and attributed to the elastic limit. In our case, the strain caused by the discontinuity of $60^{\circ}$ between the domains is relaxed by the twisting between different layers (domains). The reason for the presence of the $\sim 60^{\circ}$ critical angle in our system, however, is not yet understood.

Structures similar to the monolayer structure with periodicity in both directions, as shown in figure 5.2(C), have been observed with scanning electron microscopy (SEM) in the smectic layers formed from dry sol sediments of needle-like $\beta$-FeOOH particles ${ }^{17}$ (fig.5.5 (B)), which are well known for forming a smectic phase in aqueous suspension. The $\beta$-FeOOH particles are typically $300-500 \mathrm{~nm}$ long and tens of nanometers wide.

The similarity between the CdSe superlattices and those of TMV and $\beta-\mathrm{FeOOH}$ particles suggests that liquid crystalline phases may have formed prior to the complete evaporation of the solvent. This is not surprising, because it has been well known that dispersion of rigid rods can form lyotropic nematic and smectic phases if the rods have 
large enough aspect ratio and they are dispersed in a solvent to a high enough concentration $^{18} 19$.

By assuming the formation of the liquid crystalline phases, we can qualitatively understand the formation of the superlattices in figure 5.2 and 5.3. For example, the formation of the vortex structures can be attributed to the crystallization around disclinations in a nematic phase (see Chapter 6). According to Onsager's rigid rod theory ${ }^{18}$, when the solution is very concentrated, the Frank constants for splay and for bend become much larger than that for twist ${ }^{20}$. Therefore during the drying in the vicinity
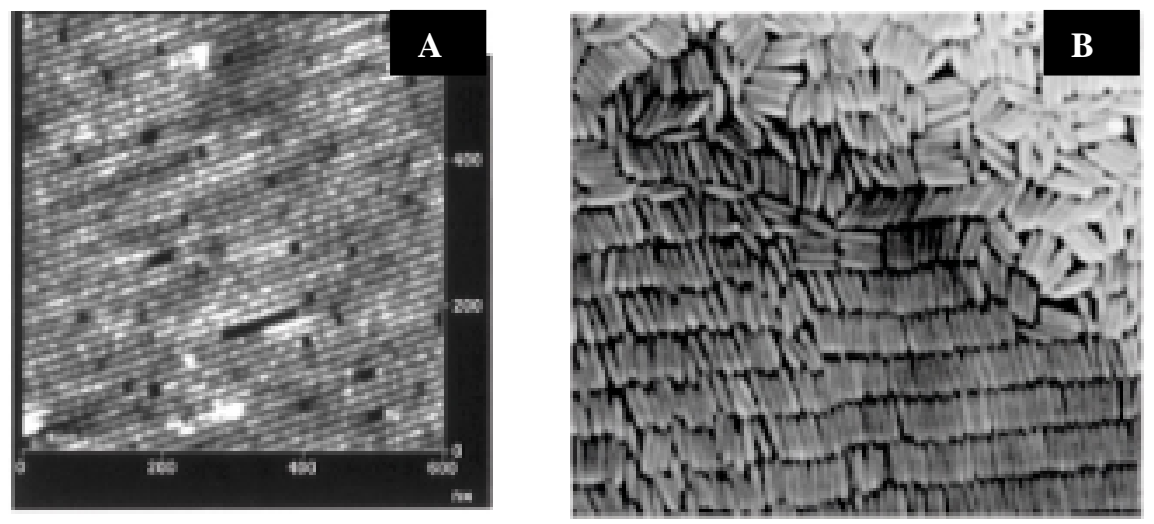

Fig. 5.5 Superlattices formed in rodlike tobacco mosaic virus (TMV) ${ }^{16}$ (A, AFM image) and $\beta$ $\mathrm{FeO}(\mathrm{OH})^{17}$ (B, SEM image) (B) during the slow solvent evaporation. Both have been well known for the formation of lyotropic liquid crystalline phases.

to the vortices, the nanorods break into different layers. The discontinuities in orientation appear across the boundaries of two neighboring branches, in order to lower the total elastic energy. As a result, each branch around a defect is a distinct crystalline domain, so we conclude that the cores of the vortices should be the nucleation sites for the linear arrays. 
These results encourage us to go on to make the stable lyotropic liquid crystalline phases of CdSe nanorod solution. As described in the following chapters, we have indeed observed the nematic phase, and have been able to align the nanorods on large scales by taking advantage of the long-range orientational correlation in the liquid crystalline phases. However, so far we do not have any direct evidence of smectic phases in CdSe nanorod solution, despite the superlattice structures we have observed, which suggest their existence. 


\section{Reference}

${ }^{1}$ Israelachvili, J. N. Intermolecular and Surface Forces, Academic Press: London, 1985.

${ }^{2}$ Murray, C. B.; Kagan, C. R.; Bawendi, M. G. Science 1995, 270 (5240), 1335-1338.

${ }^{3}$ Kagan, C. R.; Murray, C. B.; Nirmal, M.; Bawendi, M. G. Phys. Rev. Lett. 1996, 76 (9), 1517-1520.

${ }^{4}$ Dabbousi, B. O.; Murray, C. B.; Rubner, M. F.; Bawendi, M. G. Chem. Mat. 1994, 6 (2), 216-219.

${ }^{5}$ Collier, C. P.; Saykally, R. J.; Shiang, J. J.; Henrichs, S. E.; Heath, J. R. Science 1997, 277 (5334), 1978-1981.

${ }^{6}$ Nikoobakht, B.; Wang, Z. L.; El-Sayed, M. A. J. Phys. Chem. B 2000, 104 (36), 86358638.

${ }^{7}$ Kim, F.; Kwan, S.; Akana, J.; Yang, P. J. Am. Chem. Soc. 2001, 123 (18), 4360-4361.

${ }^{8}$ Li, M.; Schnablegger, H.; Mann, S. Nature 1999, 402 (6760), 393-395.

${ }^{9}$ Jerome, B. Rep. Prog. Phys. 1991, 54 (3), 391-451.

${ }^{10}$ Wei, X.; Hong, S.-C.; Zhuang, X.; Goto, T.; Shen, Y. R. Phys. Rev. E 2000, 62 (4), 5160-5172.

${ }^{11}$ Berreman, D. W. Phys. Rev. Lett. 1972, 28 (26), 1683-1686.

${ }^{12}$ Wittmann, J. C.; Smith, P. Nature, 1991, 352 (6334), 414-417.

${ }^{13}$ Li, L.-S., Alivisatos, A. P. Adv. Mat. 2003, 15 (5): 408-412.

${ }^{14}$ Denkov, N. D.; Velev, O. D.; Kralchevsky, P. A.; Ivanov, I. B.; Yoshimura, H.; Nagayama, K. Nature 1993, 361 (6407): 26-26.

${ }^{15}$ Chandrasekhar, S. Liquid Crystals, Cambridge Univ. Press: Cambridge 1992, 119.

${ }^{16}$ Maeda, H. Langmuir 1997, 13 (15): 4150-4161. 
${ }^{17}$ Maeda, H.; Maeda, Y. Langmuir 1996, 12 (6): 1446-1452.

${ }^{18}$ Onsager, L. Ann. N. Y. Acad. Sci. 1949, 51, 627-659.

${ }^{19}$ Frenkel, D.; Lekkerkerker, H. N. W.; Stroobants, A. Nature 1988, 332 (6167), 822823.

${ }^{20}$ Straley, J. P. Phys. Rev. A 1973, 8, 2181-2183. 


\section{Chapter 6. Lyotropic Liquid Crystalline Phase of CdSe Nanorod Solutions}

\subsection{Motivation}

One very common phenomenon among molecules or particles with anisotropic shapes (e.g. rodlike, disclike, or banana shaped) is the formation of liquid crystalline phases (or mesophases). A characteristic of liquid crystals is the high manipulability of their orientation. That is, their orientation can be controlled by external (electric and magnetic) fields and pretreated surfaces, due to the high cooperativity of the components. As discussed in the chapter 5, the formation of the CdSe nanorod superlattice structures similar to those observed in tobacco mosaic viruses and $\beta-\mathrm{FeOOH}$ colloids suggests that liquid crystalline phases may have formed in CdSe nanorod solution before it is completely dried. In this chapter, we go ahead to explore this possibility and prove that it is indeed the case for the CdSe nanorod solution to have a liquid crystalline phase, when it is concentrated enough. We use an optical polarizing microscope and x-ray diffraction to study the order in concentrated CdSe nanorod solutions, and confirm the presence of a nematic phase. 


\subsection{Liquid Crystals}

Liquid crystals have been an intensively studied subject, because of the presence of long-range orientational order but partial or no positional order between the liquid crystal forming molecules (or mesogens, mesogenic molecules), and their highly sensitive response to external fields ${ }^{1}$. The molecules in liquid crystals have considerable freedom to move, so that liquid crystals are more "liquid" than "solid".

Depending on the extent of orders in the liquid crystals, the mesophases formed from rodlike achiral mesogens are generally divided into two types: nematic and smectic.

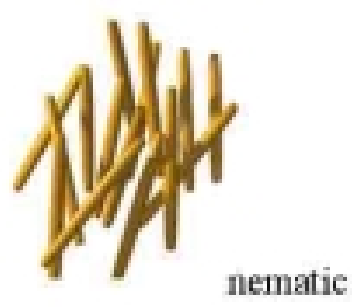

Fig.6.1 A snapshot of the arrangement of mesogen molecules in a nematic and smectic-A phase.

Figure $\quad 6.1$ schematically shows snapshots of the molecular arrangement in these two phases. In the nematic smectic-A phase, the molecules on average have more or less the same orientation but no positional order, while in smectic phases they are arranged in layers in addition to the orientational order. As a result, small angle x-ray scattering is the only direct method to identify the nematic and smectic phases, because it measures the microscopic positional and orientational arrangement of the mesogens.

\subsubsection{Line Defects in Liquid Crystals - Disclinations}

Different types of mesophases display characteristic patterns when observed between two crossed polarizers due to the constraint of the microscopic order on the possible 
distortions, and therefore can be used as an indirect way to identify the type of the mesophases. For a nematic phase, three types of deformations are allowed: splay, twist

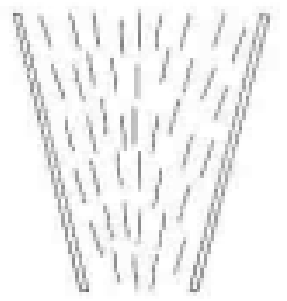
splay

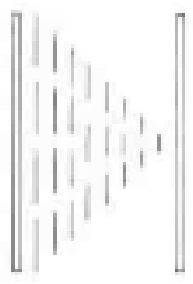

twist

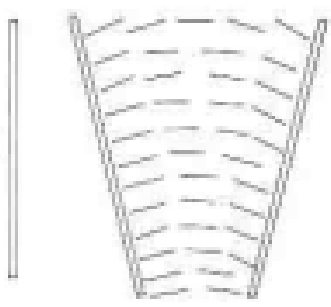

bend

Fig. 6.2 The allowed distortions in a nematic phase.

The bars represent the rod-like mesogenic molecules ${ }^{1}$.

and bend, as shown in figure 6.2, while in smectic phases only splay is present, due to the restriction of their stratified structures ${ }^{1}$. Formation of all distortions involves work. The energy involved is described as elastic energy in the continuum theory, and the elastic constants are

called Frank constants. The possible distortions determine the microscopic arrangement of the molecules around the defects, which in turn are reflected macroscopically, especially between two crossed polarizers, e.g. thread-like structures (Schlieren) in nematic phases and focal conic patterns in smectic phases.

The most common defects in liquid crystalline films are those "line singularities" around which the orientations of the directors are not well defined. Since the molecules around them tend to relax in a continuous fashion to minimize the elastic energy, between two crossed polarizers, dark brushes are observed sticking out from singular points. While the dark points are the line singularity normal to the films, the dark brushes are regions where the director (or local optical axis) is parallel to either of the two polarizer directions. These line defects are called "disclinations", in distinction to the dislocations in crystalline solids. Disclinations may have different strengths such as $\pm 1 / 2, \pm 1, \pm 3 / 2$, \pm 2 , etc., as defined by the numbers of the dark brushes sticking out of the disclinations 
divided by 4 . The strengths are also defined as the numbers of times the directors turn along an arbitrary closed curve around the line defects. Figure 6.3 illustrates the arrangement of the directors around the disclinations with different strengths in a nematic
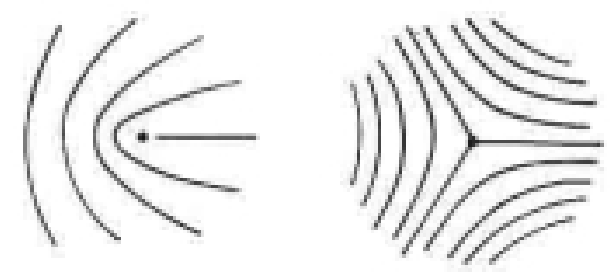

$$
S=1 / 2
$$$$
S=-1 / 2
$$

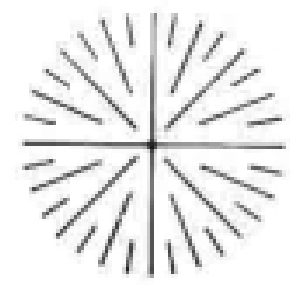

$$
\mathrm{S}=\mathbf{1}
$$

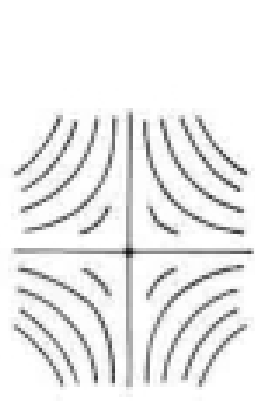

$$
S=-1
$$

phase.

In a nematic phase, under the approximation that the Frank constants for three types of allowed distortions are equal, the elastic energy involved in the disclinations is quadratic to the strength, and therefore the disclinations with larger strengths have higher elastic energy.

Fig. 6.3 The schematics of the arrangement of the molecules around disclinations of different strengths ${ }^{1}$.

\subsubsection{Lyotropic Liquid Crystalline Phases}

Based on the parameters governing their phase behaviors, liquid crystals can be divided into lyotropic and thermotropic ones. Lyotropic liquid crystals are formed by dissolving geometrically anisotropic substances in a non-mesogenic solvent, and have mainly concentration-dependent (in addition to temperature-dependent) phase behavior; while thermotropic ones include those mesogenic pure compounds and their mixtures, which display primarily temperature induced phase transitions. 
Only when the concentration of the solute is higher than a certain threshold can a lyotropic liquid crystalline phase form. It has been well known that macromolecules, viruses, and even colloidal particles, if highly anisotropic in shape and dispersed in some solvent to a high enough concentration, can form liquid crystalline phases. Good examples are poly $\left(\gamma\right.$-benzyl $\alpha$, L-glutamate) $(\mathrm{PBLG})^{2}, \mathrm{DNA}^{3}$, Tobacco Mosaic virus ${ }^{4}, \mathrm{fd}$ virus ${ }^{5}, \mathrm{~V}_{2} \mathrm{O}_{5}$ nanoribbons, and $\beta$ - $\mathrm{FeO}(\mathrm{OH})$ colloids $^{6}$ etc.

\subsubsection{Onsager's Theory - Hard Rod Model}

In 1949, Onsager proposed a "hard rod model" 7 to account for the existence of orientational order in tobacco mosaic viruses solution. He studied the suspension of cylindrical objects with length $l$ and width $d$, and assumed that the potential between them equals zero unless they overlap, where the potential goes to positive infinity. For very long rods $(l / d>100)$, he obteined the free energy of the solution up to the second virial coefficient as ${ }^{8}$

$$
\frac{\Delta F}{N k T}=\frac{\mu^{0}}{k T}+\ln \left(\Lambda^{3} \rho\right)-1+\int f(\Omega) \ln [4 \pi f(\Omega)] d \Omega+\frac{1}{2} \rho \iint v_{\text {exclusive }}\left(\Omega, \Omega^{\prime}\right) f(\Omega) f\left(\Omega^{\prime}\right) d \Omega d \Omega^{\prime}+\ldots
$$

where $f(\Omega)$ is the orientational distribution function of the rods in a 3-dimentional space. The two integrals on the right hand side of the equation have very simple physical meanings. If rods with different orientation are considered to be different species, the first integral is mixing entropy (also called orientational entropy), and the second integral gives the packing entropy, or the configuration numbers that the rods do not penetrate 
each other. The orientational entropy favors a completely random orientational distribution, while the packing entropy favors alignment, because for hard rods, the

exclusive volume $v_{\text {exclusive }}\left(\Omega, \Omega^{\prime}\right) \sim|\sin \theta|$, where $\theta$ is the angle between two rods. As a result these two entropy terms compete. Only when $\rho$ is high enough would the packing entropy term dominate, and an orientationally ordered phase would form. The critical concentration was determined to be $\sim 4 d / l$ for long rods, and the coexisting isotropic and nematic phases have concentrations of $\sim 3.3 d / l$ and $\sim 4.1 d / l$ with a ratio of 1.24 .

The Frank constants was evaluated in the rigid rod model ${ }^{9}$, and it was found that the bend has much larger Frank constant than splay or twist, and that the Frank constant for twist is only a third of that for splay. This is why in the vortex structures formed when the nanorods are dried (figure 5.3) the different branches tend to appear in different layers to avoid sharp bend and splay distortions.

Because of the long rod assumption, Onsager's theory is quantitatively accurate for rigid rods with aspect ratio larger than 100, and qualitatively correct for those with aspect ratio larger than 10 . Most importantly, the rigid rod model is conceptually simple and captures the essential physical picture of the formation of lyotropic liquid crystals, so it often serves as the starting point for various extensions and corrections to explain experimental results ${ }^{10}$.

\subsubsection{Flory's Theories}

Because of the singular potential assumed in Onsager's model, the dispersion of the rigid rods is athermal. In practice, however, the interaction between the rods and the 
solvent also plays important roles in determining the phase behaviors. A good example is the thermo-reversible gelation in rodlike polymer solutions when poor solvents are used. These are taken into account in the lattice model proposed by Flory ${ }^{11}{ }^{12}$. In this model, the solution of rigid rodlike particles is described as a cubic lattice with one solvent molecule or one segment of the rigid rod occupying one lattice site. The rigid rods occupy a linear series of lattice sites either along or at an angle to the principle lattice axis (an axis parallel to one dimension of the lattice), and when the solution gets concentrated it becomes necessary to align the rods into the lattice.

Flory characterized the orientation of a rigid rod-like polymer molecule with an angle from the principle lattice axis. Each rigid rod is divided into $y$ subunits of length $x / y$ parallel to the axis and connected to the neighboring subunits at its end points, where $x$ is the aspect ratio of the rods. The parameter $y$ is the measure of both the disorder in the system and the length of each subunit parallel to the axis $(x / y)$. When the system is completely disordered, $y=x$, and $y=1$ for a complete order.

The partition function is built up by calculating the combinatory part for the rigid rod to fit into the lattice without overlapping each other and the orientation part. The free energy of mixing between the solvent and the rod-like solute is expressed as:

$$
\begin{gathered}
\Delta G_{M} / k T=n_{1} \ln v_{1}+n_{2} \ln v_{2}-\left(n_{1}+y n_{2}\right) \ln \left[1-v_{2}(1-y / x)\right] \\
-n_{2}\left[\ln \left(y x^{2}\right)-y+1\right]+\chi x n_{2} v_{1}
\end{gathered}
$$

where $n_{1}, n_{2}$ are the number of solvent molecules or polymer molecules, $v_{1}, v_{2}$ their volume fractions, $\chi$ the polymer-solvent interaction parameter, and $y$ the degree of disorder. 
For the ordered phase, $\Delta G_{M}$ is minimized with respect to $y$ to yield

$$
v_{2}=[x /(x-y)]\{1-\exp (-2 / y)\}
$$

which has a solution only when $v_{2}$ is above a critical value $v_{2}^{*} \approx \frac{8}{x}\left(1-\frac{2}{x}\right)$.

According to the lattice model, the phase diagram for rods with a given aspect ratio can be calculated by equating the chemical potentials of the solute and the solvent in the ordered and disordered phases with the equation (2), respectively, as shown in figure 6.4 .

The polymer-solvent parameter $\chi$ is a dimensionless quantity related to the mixing enthalpy ${ }^{13}$ of the solute and the solvent (a negative $\chi$ means an exothermic mixing), and it is roughly linear to the reciprocal of temperature. Therefore the phase diagram shown in figure 6.4

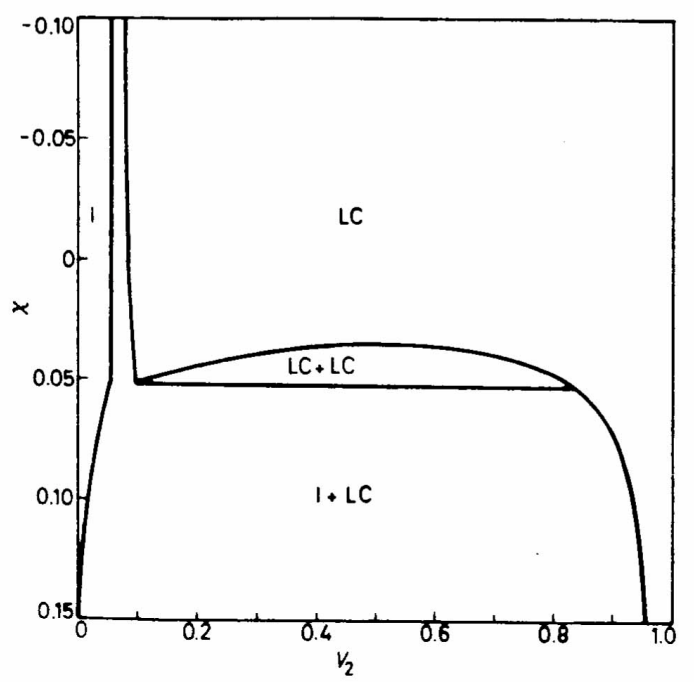

Fig. 6.4 Phase diagram predicted by the Flory's model for rigid rods of aspect ratio of $150^{11}$.

indicates the presence of the temperature dependence.

The anisotropic shape results in orientational van der Waals interaction. It was found that adding the "soft" interaction between the rods only slightly refine the calculated phase diagram, indicating the "hard" interaction between the rods is far more important in lyotropic liquid crystals. 


\subsection{Spontaneous Optical Anisotropy in CdSe Nanorod Solution}

The formation of liquid crystalline phases of the CdSe nanorods was verified by examining birefringence in solutions of nanorods under an optical microscope with crossed polarizers. The change in the polarization of the incident beam by an optically anisotropic medium makes this technique a powerful tool to distinguish an isotropic medium from an anisotropic one. Furthermore, addition of a rotating sample stage makes it possible to determine the 2-dimentional projection of the optical axes of the anisotropic medium, e.g., when the optical axes are parallel to either of the direction of the polarizers, the extinction of the transmitted light is observed. Polarizing optical microscopy is widely used in the study of anisotropic matters. In particular, the unique defect structures observed in different liquid crystalline phases make it possible to distinguish nematic and smectic phases.

A solution of CdSe nanorods (in cyclohexane, 10\% wt.) with a length of $40.0 \mathrm{~nm}$ and a width of $4.0 \mathrm{~nm}$ is sealed in a capillary tube. The solution itself is isotropic at such a low concentration. When it is mounted on the microscope, the heating caused by the incident light slowly evaporates the solvent at the focusing spot, causing the solution to become more concentrated, leading to the formation of a birefringent liquid crystalline phase ${ }^{14}$. The anisotropic refractive index of the liquid crystalline phase alters the polarization of transmitted light, leading to light and dark patterns on the micron scale. Because the capillary tube is sealed and the light only changes the distribution of solvent within the tube, we can change the local concentration of the solution by varying the intensity of incident light, and we can also control the speed of evaporation and monitor the evolution of the liquid crystalline phase reversibly. Figure 6.5 shows the birefringent 
pattern observed at two different stages. Isotropic liquids show complete extinction, and no pattern is observed from concentrated solutions of mixtures of the two surfactants used in the synthesis of these nanorods (trioctylphosphine oxide and tetradecylphosphonic acid). The intensity of the transmitted light reveals the orientation of the nanorods.
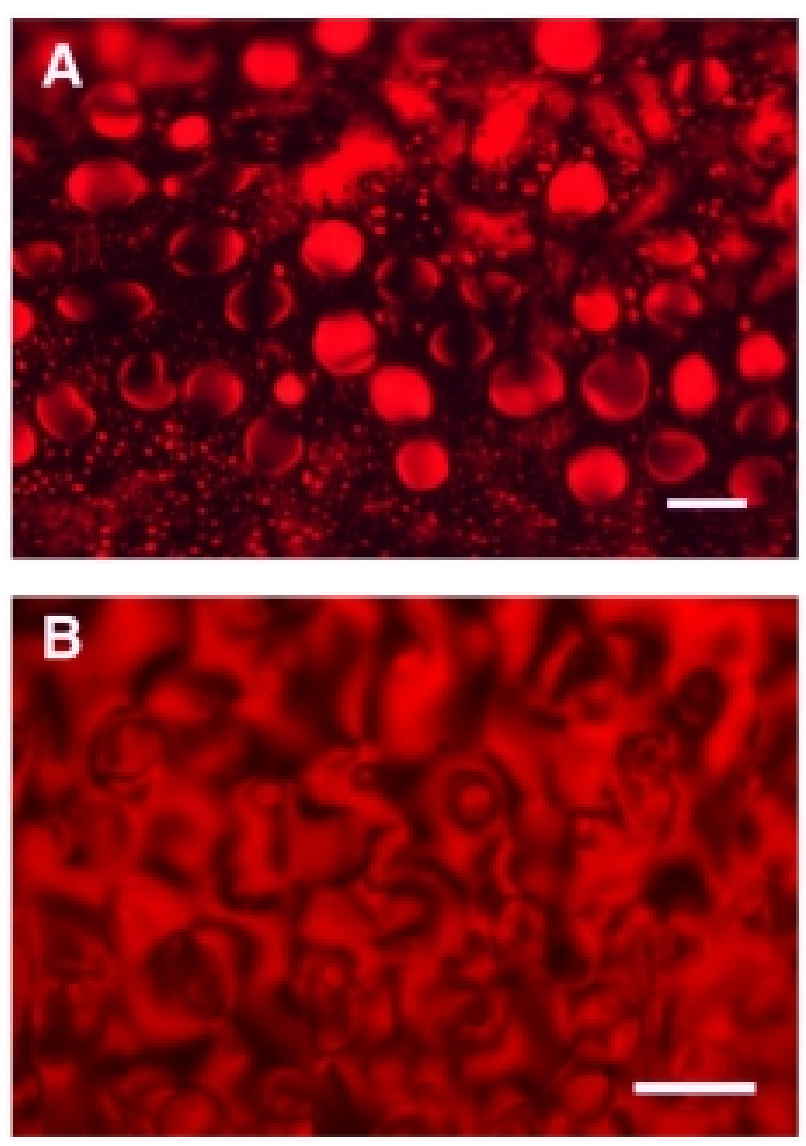

Fig. 6.5 Birefringence observed under a polarizing optical microscope during evaporation of solvent $^{14}$. (A) Droplets (tactoids) formed from the isotropic phase with the evaporation of solvent. (B) Schlieren textures in a later stage. The red background is due to the absorption spectra of CdSe nanocrystals. The scale bars are $40 \mu \mathrm{m}$.

Figure 6.5(A) shows the nucleation of the liquid crystalline phase. Small $(\sim 2 \mu \mathrm{m})$ and large $(\sim 10-20 \mu \mathrm{m})$ birefringent droplets (tactoids) emerge from an isotropic solution as the concentration increases. The solution appears red because of the band gap of the CdSe nanocrystals. As they have higher optical density than the isotropic medium we conclude that these droplets have higher concentration of nanorods. The variations in the 
intensity of the transmitted light through the droplets indicate the non-uniform alignment of the rods within each droplet, because only when the droplets are optically anisotropic and their local optical axes are parallel to neither of the polarizer axes would there be transmitted light. When the nanorods are aligned in each droplet, the bending of the droplet surface must require different amount of work, depending on whether the bending is parallel or perpendicular to the nanorods. The slightly elongated non-spherical shape of the droplets provides another clear indication that the nanorods are oriented within each droplet, as a result of the balance between surface tension and anisotropic elasticity, in which the former favors a spherical surface while the latter favors surface to be straight, or the surface behaves as if it possesses an anisotropic surface tension ${ }^{15}$. Therefore the surface of the droplets, as shown in figure 6.6, is determined by the equation in the form of Laplace equation, $\frac{\sigma_{1}}{r_{1}}+\frac{\sigma_{2}}{r_{2}}=$ constant, and the requirement that the surface energy of the given volume is minimized, where $\sigma_{\mathrm{i}}$ and $r_{\mathrm{i}}(\mathrm{i}=1,2)$ are the principle values of the surface tension and curvature radii of the interface, respectively.

Once the liquid crystalline phases have fully developed, a new set of patterns is observed. Figure 6.5(B) shows the Schlieren structures formed at a later stage. Dark threadlike brushes are observed sticking out from some singular points. The singular points (disclinations) are the positions where the alignment direction of particles is not well defined. The dark brushes are due to the particles with alignment parallel to

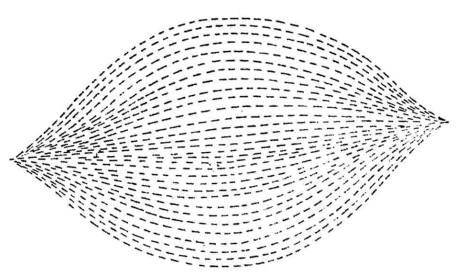

Fig. 6.6 Schematic of the orientations of the nanorods in a tactoid, with the bars representing the $\operatorname{rods}^{15}$. 
either of the polarizers. When the sample is rotated, these singular points remain fixed but the dark brushes move around them. When one end of the capillary tube is lifted the patterns flow and deform easily, suggesting the fluidity of the birefringent phase. Similar observations were also reported in colloidal boehmite rod suspensions ${ }^{16}$.

\subsection{Stable liquid crystalline solution of CdSe Nanorods}

Despite the observation of optical anisotropy in the CdSe nanorod solution during the solvent evaporation, the unambiguous identification of the birefringent substance to be a liquid crystalline phase requires it to be isolated and subsequently characterized. However, making a stable birefringent solution is not trivial, because of the kinetics of the phase separation.

\subsubsection{Thermal-reversible Gelation in Concentrated Solution of CdSe Nanorods}

The most serious obstacle to prepare stable birefringent solution of CdSe nanorods is the gelation when the solution is successively concentrated, which has been commonly seen in rodlike polymer systems, and is believed to be related to the mechanism of the phase separation $^{12}{ }^{17}$.

\subsubsection{Phase Separation Mechanisms and Gelation}

Figure 6.7.A shows a phase diagram for a regular solution. The thermodynamic boundary separating different phases are called bimodal curve, above which the system appears as a homogeneous solution. Inside the bimodal curve is the biphasic region, and 
the system is thermodynamically stable if it separates into two phases. The biphasic region is further divided into a metastable region (between the bimodal and the spinodal curves) and an unstable region (inside the spinodal curve) by the spinodal curves, which is mathematically defined as the points with $\left(\frac{\partial^{2} G}{\partial x^{2}}\right)_{T}=0$, where $G$ stands for the Gibbs free energy of the system, $x$ the composition and $T$ the temperature.

Figure 6.7.B shows the schematics of the Gibbs free energy of the system as the function of the composition at different temperatures. For a temperature $T_{0}$ higher than the critical temperature $T_{c}$, the free energy has a unique global minimum, which corresponds to a single homogeneous solution. For a temperature $T_{1}$ lower than $\mathrm{T}_{\mathrm{c}}$, however, the free energy has two minima at the composition of the two separate phases (i.e.
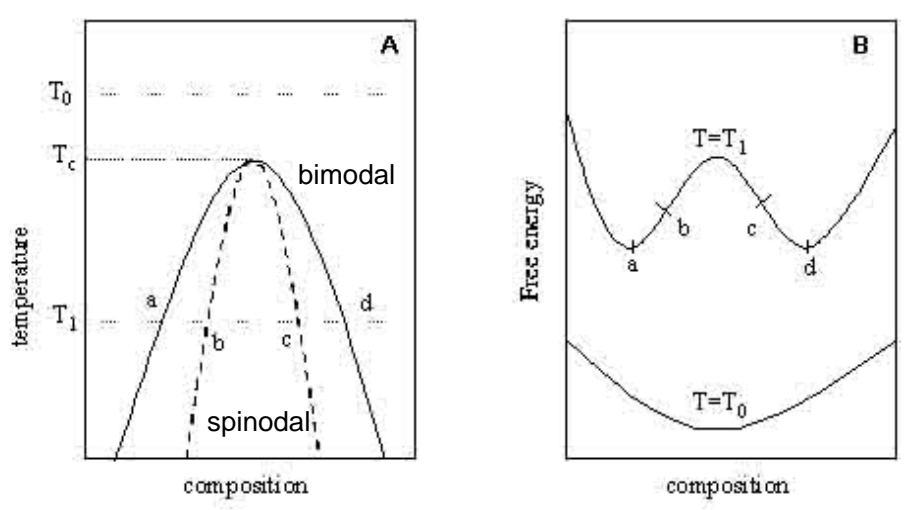

Fig. 6.7 A) Temperature-composition phase diagram of a regular solution. The sold line is the bimodal curve, the thermodynamic boundary separating the biphasic from the single phase regions. The dashed line is the spinodal curve, separating the metastable from the unstable regions. $T_{c}$ is the critical temperature.

B) The schematic of the free energy of the mixture with different composition at two temperatures $\left(\mathrm{T}_{0}>\mathrm{Tc}\right.$ and $T_{1}<T_{c}$ ). The free energy of the whole system is the summation of the free energy of the different parts weighed by volume if the system is not homogeneous.

point $a$ and $b$, which define the bimodal curve). The inflection points $c$ and $d$ defines the 
spinodal curve at $\mathrm{T}_{1}$, for they satisfy $\left(\frac{\partial^{2} G}{\partial x^{2}}\right)_{T}=0$. When the overall concentration of the system sits between $a$ and $b$, or $c$ and $d$, any continuous change in the composition will result in an overall increase in the free energy of the system due to the positive curvature. Similarly, for a system with composition between $c$ and $d$, continuous change in the composition is accompanied by the lowering overall free energy, which suggests a continuous phase separation mechanism.

The phase separation for a system in the metastable region, which is separated from the global free energy minimum by an energy barrier, is governed by a nucleation-andgrowth mechanism; while for a system within the spinodal region, the phase separation governed by the spinodal decomposition mechanism. Figure 6.8 schematically shows the evolution of the composition profiles for both cases. In the nucleation-and-

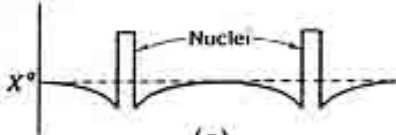

(a)

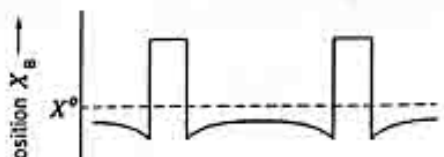

(b)

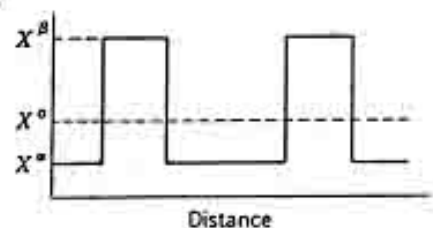

(c)

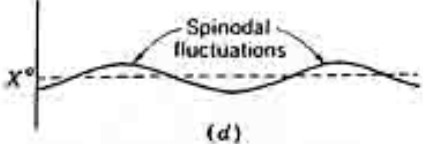

(d)

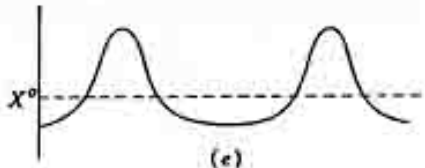

(e)

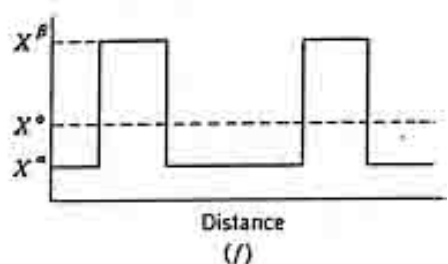

(f)

Fig. 6. 8 Composition distribution in phase separation following nucleation-and growth mechanism and spinodal decomposition mechanism, respectively ${ }^{12}$.

extrema of concentration exist

from the very beginning, and the amount of the two phases increases with time. In the spinodal mechanism, the local concentration in the system continuously deviates from the average until complete phase separation is achieved. 
Both mechanisms result in the same thermodynamical configuration, as defined by the bimodal curve. However, the difference in the resulting morphology is dramatic. While for the nucleation-and-growth mechanism, the final morphology is determined mainly by the nucleation events; the spinodal decomposition results in 3-dimensional, bicontinuous fractal network, as predicted by theoretical calculation ${ }^{18}$.

Flory's lattice theory was extended to calculate bimodal and spinodal curves for polymer solutions ${ }^{12}$, and the spinodal decomposition is often believed to be the mechanism that results in gelation in the polymer solutions. During the phase separation, spinodal decomposition requires that the polymer molecules diffuse into areas with higher concentration (i.e. negative diffusion constant), which results in gels with high local viscosity and low fluidity. The gelation makes the complete isotropic-liquid crystalline phase separation a very slow process, and consequently the system can remain in such nonequilibrium for extended period of time.

Studies on the gels formed from the rod-like polymers such as PBLG by suddenly reducing the solvent quality (by decreasing the temperature or adding poor solvent to the solution $)^{12}$ confirm the network structure and the negative diffusion constant, as well as the liquid crystal formation just before gel melting, and therefore the network structure is often believed to be indications of spinodal decomposition. 


\subsubsection{Gelation in Concentrated CdSe Solution}

Like in rodlike polymers, thermo-reversible gelation was also observed in the concentrated solution of CdSe nanorods, which is tentatively attributed to the spinodal decomposition mechanism. For example, when regular (instead of anhydrous) organic solvents, such as chloroform, toluene, cyclohexane and hexanes, are used to dissolve the CdSe nanorods to a high concentration, the solution gels after the homogenization. Gentle heating can melt the gel, which will form again when cooled down. Furthermore, if the gel is kept long enough the Schlieren patterns would form. Similar observation was also made in the solution of PBLG, and was attributed to the hindered formation of the liquid crystalline phase due to the decrement in the diffusion constant in a gel. However, to definitely prove the spinodal decomposition mechanism, low angle light or x-ray scattering measurements have to be performed and compared with the theoretical expectations for the early and later stages ${ }^{17} 1819$.

\subsubsection{Stable liquid crystalline solution of CdSe Nanorods}

Since the gelation can be attributed to the poor solubility of the nanorods in the solvents, improvement of the solvent quality may avoid gelation, as predicted by the Flory's model. In our system, it has been proved to be the case when anhydrous cyclohexane or hexanes (but not chloroform or toluene) is used as the solvent to dissolve the CdSe nanorods.

In order to avoid the exposure to water vapor in air, CdSe nanorods are dissolved in excess of anhydrous cyclohexane in a glove box filled with argon and centrifuged to get 
rid of any insoluble substances. The solution is then transferred to a $3 \mathrm{~mm}$ diameter (NMR) glass tube with pipettes, and subsequently concentrated by slowly flowing dry nitrogen. The solution is kept homogeneous throughout the drying process by repetitively turning off and on the nitrogen flow. Above a certain concentration, birefringent droplets appear and the solution becomes turbid. After being settled overnight, a translucent phase appears at the bottom of the tube with a distinct boundary to the top part of the mixture, which is still turbid. When observed between crossed polarizers, the bottom phase is highly birefringent, while the top mixture contains birefringent droplets suspended in a dark background. Figure 6.9 shows the phase separation in a concentrated solution of CdSe nanorods observed under an optical polarizing microscope. After settling for months, the bottom phase is highly birefringent and the top phase isotropic, while both phases flow when tilted. When the solution is diluted with solvent the liquid crystalline domains disappear, and the solution becomes isotropic. Shaking the solution will cause flow birefringence, which

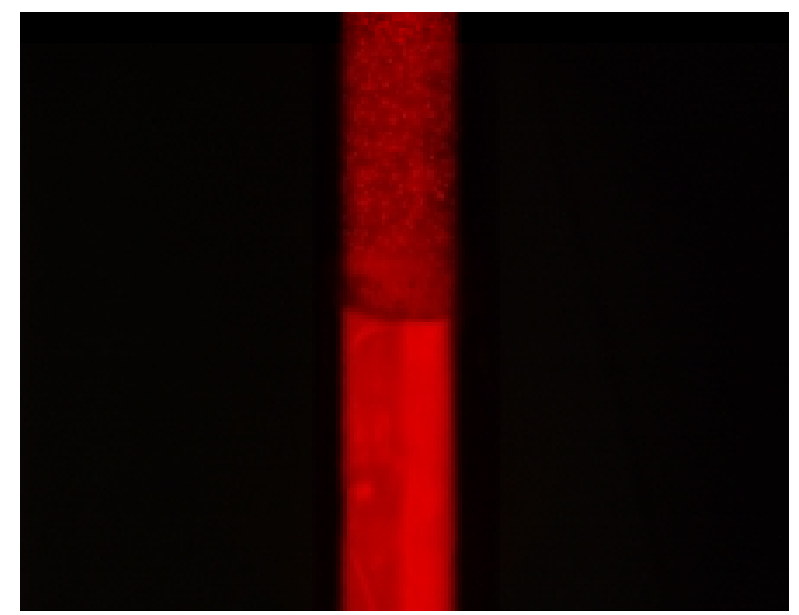

Fig. 6.9 Isotropic-liquid crystalline phase separation in a concentrated solution of CdSe nanorods in anhydrous cyclohexane. The liquid crystalline phase is denser and therefore heavier and stays at the bottom. Birefringent droplets are still seen suspending in the top isotropic phase. The nanorods are $3.0 \mathrm{~nm}$ wide and $60 \mathrm{~nm}$ long. slowly vanishes when at rest. 
Both wide-angle and small angle x-ray scattering (SAXS) were performed on aligned liquid crystalline samples in capillary tubes, which unambiguously indicate that the nanorods form a liquid crystalline phase ${ }^{20}$. Figure 6.10 shows the wide-angle $\mathrm{x}$-ray diffraction (A) and small angle x-ray scattering patterns (B) from the birefringent part of the solution as shown in figure 6.9 in a $300 \mu \mathrm{m}$ thick capillary tube. These nanorods have a length of $\sim 60 \mathrm{~nm}$ and width of $3.0 \mathrm{~nm}$. In both figures the capillary tube remains vertical. In figure 6.10 (A) the sharp arcs in the vertical position are due to the (002) planes of the CdSe crystalline lattice within individual nanorods. The high intensity and sharpness of the arcs are caused by the higher number of the (002) planes in the CdSe nanorods as a result of their elongation along the $c$-crystallographic axis. The anisotropy of the pattern demonstrates macroscopic alignment of the long axis of the rods. Consistently, in figure 6.10(B) the diffuse arcs in the equator direction correspond to the lateral distance of the nanorods, suggesting the short axis is aligned as well. Together, the patterns demonstrate the preferential alignment of the CdSe nanorods along the capillary tube axis, which is attributed to the curved wall of the capillary tube. The long-range orientational correlation between the nanorods and their tendency to minimize the elastic energy caused by the curved wall require the nanorods align along the axis of the capillary tube. This alignment effect caused by the tubes is found to be stronger in thinner capillary tubes than in thicker ones as expected. From the patterns in figure 6.10, it can be estimated that about $70 \%$ of the nanorods are oriented within $20^{\circ}$ from the axis of the capillary tube, by integrating the intensity azimuthally. However, due to the resolution of the instrument, the current result cannot identify whether it is a nematic or smectic phase, 
and therefore we can only use the disclination patterns under a polarizing microscope to determine the phase of our sample.
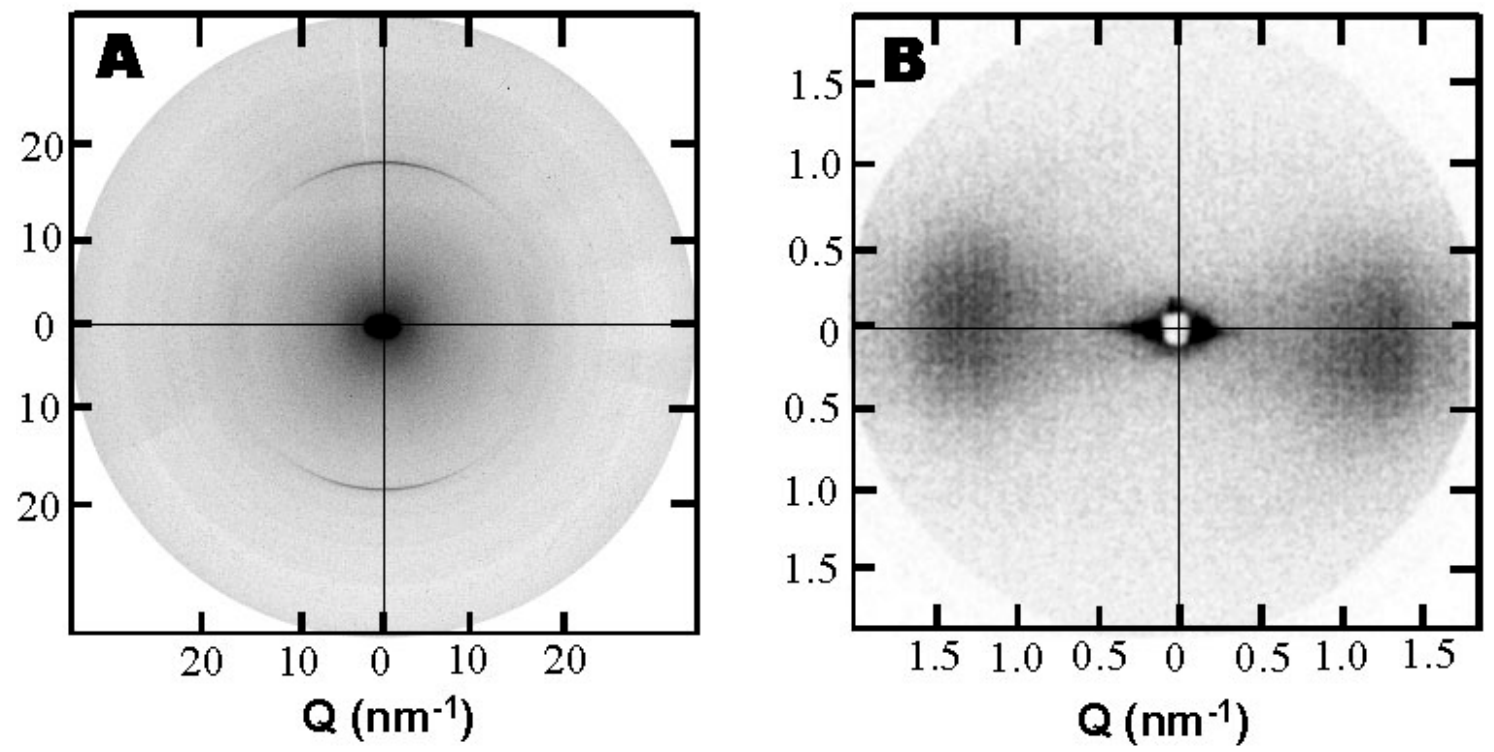

Fig. 6.10. Wide angle $x$-ray diffraction and small angle $x$-ray scattering of the same nematic solution of CdSe nanorods in a $300 \mu \mathrm{m}$ thick capillary tube show the preferential alignment of these nanorods along the axis of the capillary tube ${ }^{20}$. In both measurements the capillary tube is held vertically. A) Wide angle x-ray diffraction pattern. The vertical sharp, intense arcs correspond to the (002) planes of wurzite CdSe lattice. At about the same radius weak arcs corresponding to the (100) and (101) planes can also be seen, but they are much more diffuse. B) Small angle x-ray scattering pattern. The two diffuse arcs at $\mathrm{Q} \sim 1.5 \mathrm{~nm}^{-1}$ on the equator corresponds to the lateral spacing between the rods in the nematic phase. The arcs corresponding to the longitudinal spacing are not resolved.

Figure 6.11 shows some images taken under a polarizing optical microscope from thin films of the liquid crystalline solution of CdSe nanorods, with (A) showing the solution as a mixture of a liquid crystalline droplets and an isotropic phase one day after 
homogenization. Figure (B) shows an image of the interface between the liquid crystalline phase and the isotropic phase obtained after the droplets have grown and coalesced. Schlieren structure can also be seen.
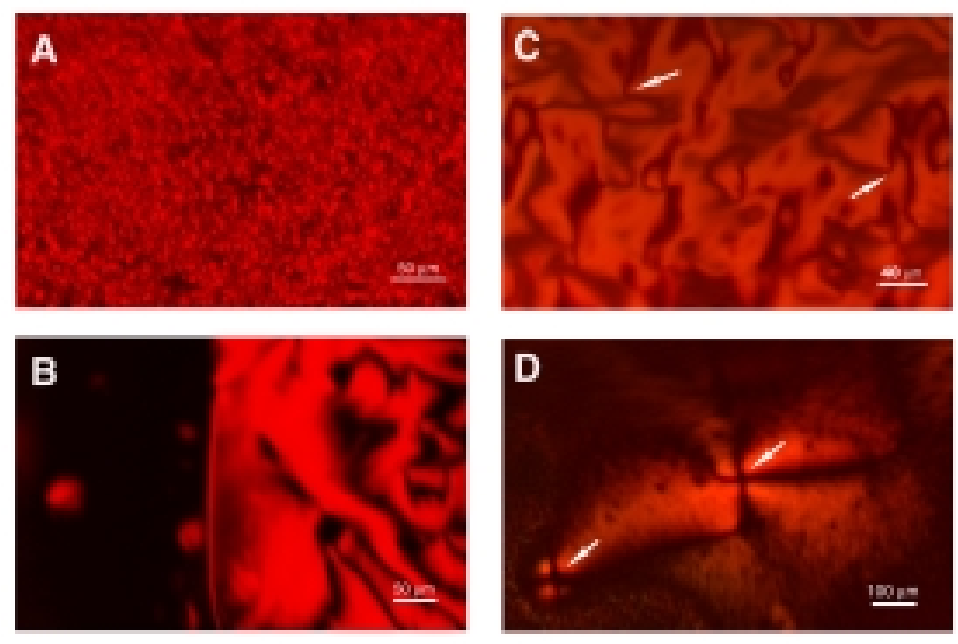

Figures $6.11(\mathrm{C})$ and (D)

Fig. 6.11 Images of liquid crystalline phase in concentrated solution of CdSe nanocrystals under a polarizing optical show some defects observed with Schlieren structures microscope. Isotropic phase appears dark in these images. All characteristic of a nematic phase. (C) shows disclinations with strength of $1 / 2$. Strength of a disclination is defined to be a quarter of the number of dark brushes the bright areas in the image correspond to the liquid crystalline phase, with dark lines corresponding to disclinations or the area where the orientation of the nanorods are parallel to either of the polarizers. (A) Droplets of liquid crystalline phase mixed with isotropic phase. (B) Interface between a big liquid crystalline droplet (right) and isotropic phase (left). (C) and (D) Disclinations of strength of $1 / 2$ (C) and 1 (D) respectively. The arrows point to the disclinations.

sticking out of it. In (D) we see disclinations with strength of 1 .

Interestingly, the disclinations with strength of 1 are observed much less often than those with strength of $1 / 2$, and sometimes even slight heating by light can anneal them out. Since the formation of disclinations in liquid crystalline phases costs elastic energy, and the energy involved increases with strength, we can conclude that the liquid crystalline 
phase of CdSe nanorods have much higher elastic constants compared to regular organic liquid crystal molecules, as expected from the high rigidity and the large size of the CdSe nanorods. These patterns indicate a nematic phase, which is yet to be proved by higher resolution SAXS experiments

\subsection{Alignment of CdSe Nanorods}

Macroscopic-scale alignment of CdSe nanorods is important for both the fundamental studies and the application of their anisotropic properties. The cooperativity between the molecules in liquid crystals enables us to alter their orientations with even subtle changes in the environment. For example, the alignment of thermotropic small molecule liquid crystals on rubbed polymers and the switching of their orientations with electric field have made the liquid crystal display devices possible. Similarly, the formation of liquid crystalline solution of CdSe nanorods should enable us to use various methods to align them. However, because the nanoparticles are much larger than the regular mesogenic molecules in size, and that the liquid crystalline solution of CdSe nanorods are very viscous and very sensitive to the water vapor in air, the application of the common methods such as pretreated surfaces, external electric or magnetic field, etc. is not straightforward.

\subsubsection{Surface induced alignment}

Despite the success in aligning thermotropic liquid crystals with rubbed polymer such as polyimide films, so far this type of the experiment for CdSe nanorods has not been successful, presumably due to the size of the nanorods defeating the mechanism for 
alignment of thermotropic liquid crystals, which is believed to be a heteroepitaxial type interaction.

On the other hand, capillary alignment of lyotropic liquid crystals has been well established $^{21}$ and has been successfully applied to CdSe nanorods ${ }^{20}$. Figure 6.10 shows the $x$-ray diffraction patterns of the nematic solution of $3.0 \times 60 \mathrm{~nm}$ nanorods in a 300 $\mu \mathrm{m}$ diameter capillary tube, which indicate a preferential alignment of the nanorods along the axis of the capillary tube.

The capillary alignment can be understood from the elastic energy point of view. Lyotropic liquid crystals generally assume planar configurations near a surface, i.e. the directors are in plane. If the surface is curved with a radius of curvature smaller than the orientational correlation length of the molecules (which is much larger than the dimension of the molecules), large elastic energy is involved due to the distortion in the liquid crystals. Thus when the lyotropic liquid crystals are confined in a thin capillary tube, the directors tend to align along the axis of the tube to lower the elastic energy.

\subsubsection{Alignment of CdSe Nanorods with Magnetic Field}

The orientational correlation in liquid crystals amplifies the anisotropy of the extensive properties of the mesogenic molecules, such as electric polarizability and magnetic susceptibility, so that the liquid crystals have very sensitive response to external fields. If, for each individual molecule, $\Delta \chi=\chi_{\|}-\chi_{\perp}$ with $\chi_{\|, \perp}$ being either electric or magnetic susceptibility along and perpendicular to the director respectively, the cooperativity between the $N$ molecules in the liquid crystals makes the total susceptibility 
anisotropy of the system $N \cdot \Delta \chi$. If we assume the magnetic susceptibility of CdSe nanorods to be the same as bulk wurtzite, i.e. $\chi_{c}=-6.77 \times 10^{-5} \mathrm{~cm}^{3} / \mathrm{mol}$ and $\chi_{a}=-5.82 \times 10^{-5} \mathrm{~cm}^{3} / \mathrm{mol}$, for a $3.0 \times 60 \mathrm{~nm}$ nanorods $\Delta \chi=-0.122 \mathrm{~A}^{3}=-1.22 \times 10^{-24}$ $\mathrm{J} /$ Tesla $^{2}$. Under a magnetic field of 1 Tesla, $\Delta E=-\frac{1}{2} \Delta \chi B^{2} \sim 0.044 \mathrm{~K}$. If $10^{6}$ nanorods are correlated, $\Delta E=44000 \mathrm{~K}$, therefore the liquid crystals can be easily aligned by the magnetic field, with the long axis perpendicular to the magnetic field.

Because of the short penetration depth of $\mathrm{x}$-ray in $\mathrm{CdSe}(\sim 10 \mu \mathrm{m})$ and the aligning effect of thin capillary tube on the nanorods, we cannot use x-ray diffraction to study the ordering of nanorods under magnetic fields. On the other hand, nuclear magnetic resonance (NMR) is a method widely used to study liquid crystalline phases ${ }^{22}$. Many quantities can be used to probe the order parameter as long as they have orientational dependence, such as magnetic dipole-dipole coupling, chemical shift anisotropy and quadruple splitting. The only disadvantage is that it requires much more sample than $\mathrm{x}$ ray diffraction measurements. Here we use quadruple splitting of deuterium nuclei in $\mathrm{CDCl}_{3}$ molecules, which are purposefully introduced to the system due to the simple interpretation of their NMR absorption spectra 232425 .

Deuterium nuclei have spin of 1 and non-zero electric quadruple. The non-spherical charge distribution coupling to the non-uniform local electric field generates a torque and cause the nuclear to precess. This is similar to the slow precession of the earth's axis as a result of the ellipticity of the earth's mass distribution and the non-uniform gravity fields from the moon and the sun. For the specific case of $\mathrm{CDCl}_{3}$, the electric field gradient 
(EFG) near the deuterium nuclear is cylindrically symmetric, and the modification of the energy associated with each of the possible orientations of the deuterium nuclear spin in a magnetic field is given by

$$
H_{Q}{ }^{(1)}=\frac{3 e Q V_{33}}{8 I(2 I-1) \hbar}\left(3 \cos ^{2} \theta-1\right)\left[I_{z}^{2}-I(I-1) / 3\right],
$$

where $Q$ is the quadruple moment of the nuclear, $V_{33}$ the principle value of the EFG along the C-D bond, and $\theta$ the angle between the C-D bond and the magnetic field. It can be easily realized that when electric quadruple interaction is considered, a splitting appears in the NMR spectra, as shown in figure 6.12. If the tumbling of the molecule is much faster than the time scale of the NMR measurement, however, in an isotropic medium, the quadruple splitting is averaged to zero because of the uniform sampling of all possible orientations by the $\mathrm{C}$-D bond giving a zero average

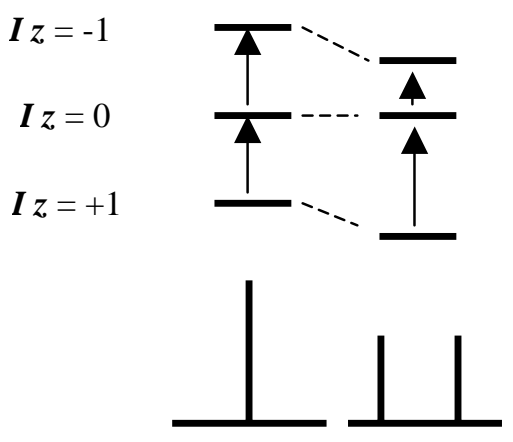

Fig. 6.12 Schematic of the transition in NMR with and without quadruple splitting.

value of the order parameter $S=\left(3 \cos ^{2} \theta-1\right) / 2$. In an anisotropic medium, such as liquid crystals, this effect does not average out and a doublet appears.

The line shape of the quadruple splitting is determined by the average orientation of the electric field gradient tensor with respect to the magnetic field. For a multi-domain sample with random orientational distribution in a 3-dimensional space, the NMR spectrum adopts the famous powder pattern (the dash line in figure 6.13$)^{23} 2425$. The 
resonance frequency is

determined by the

$\left(3 \cos ^{2} \theta-1\right)$ term, and the

corresponding intensity by

the solid angle. For a

sample in which the time

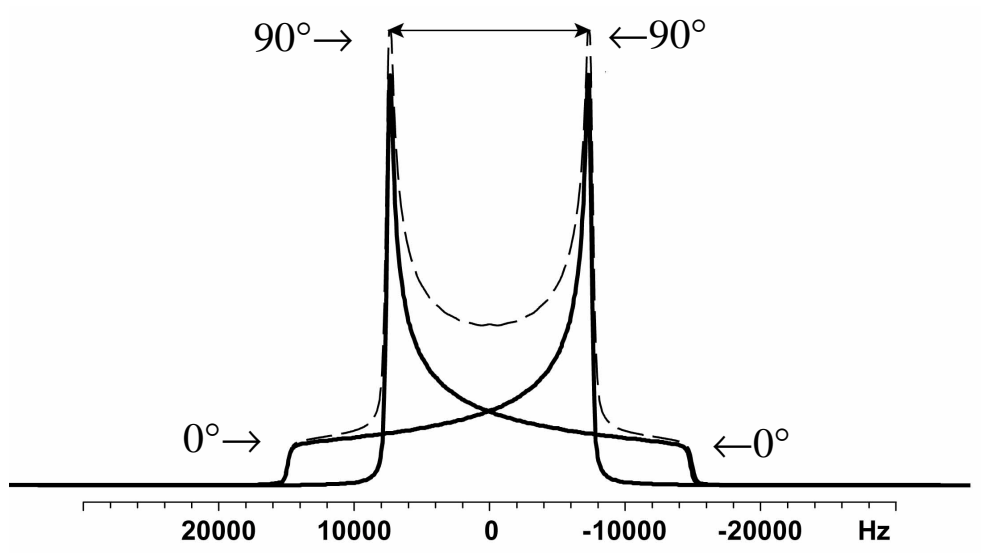

average of the EFG is

uniformly oriented with

Fig. 6.13 3-dimensional powder pattern of the quadruple splitting. It respect to the magnetic is a summation of the contribution from domains from all the orientations, arising from the $\left(3 \cos ^{2} \theta-1\right)$ dependence.

field, there would be only

two peaks, with peak

position again

determined by the

$\left(3 \cos ^{2} \theta-1\right)$ term.

To prepare the NMR

sample, $\mathrm{CDCl}_{3}$ is added

to the biphasic solution

of CdSe nanorods in

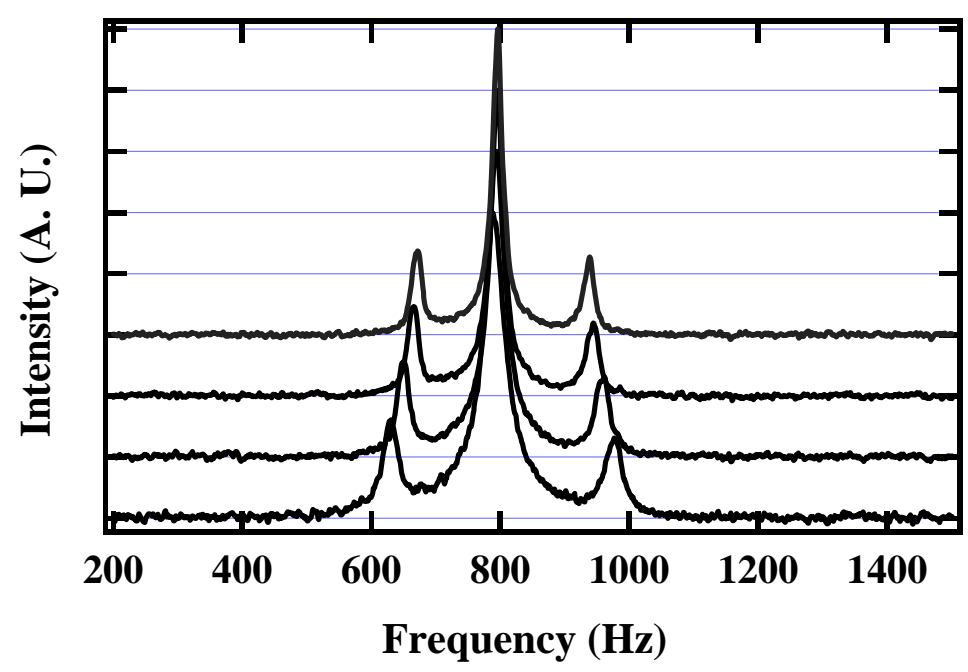

anhydrous cyclohexane

in a $4 \mathrm{~mm}$ diameter NMR

Fig. 6. 14 The D-NMR spectra of the $\mathrm{CDCl}_{3}$ in the biphasic solution of the CdSe nanorods at different temperatures (from the bottom to the top, $29^{\circ} \mathrm{C}, 47^{\circ} \mathrm{C}, 65^{\circ} \mathrm{C}, 74{ }^{\circ} \mathrm{C}$, respectively). The tube $(<5 \%$ by volume to avoid possible gelation peak widths decrease with increasing temperature due to the reduced the viscosity. 
due to the degradation of the solvent). The solution is sealed with flame. Polarizing optical microcopy is used to confirm the presence of the liquid crystalline phase. The spectra were taken in a $500 \mathrm{MHz}$ (for proton) spectrometer, and the temperature can be varied by blowing warm air through the sample position. Figure 6.14 shows the D-NMR spectra of the $\mathrm{CDCl}_{3}$ in the solution of $3.0 \times 60 \mathrm{~nm} \mathrm{CdSe}$ nanorods at different temperature. The central peak was identified to be due to the isotropic part in the sample, and the two side peaks to the liquid crystalline part.

Two features of the peaks can be easily seen form the spectra. First of all, compared with a powder pattern, the spectra indicate that the $\mathrm{CDCl}_{3}$ molecules are aligned with a uniform orientation throughout the liquid crystalline phase, presumably due to the alignment of the CdSe nanorods by the magnetic field. However, the average orientation of the nanorods cannot be determined only from the spectra here, nor can the aligning mechanism of $\mathrm{CDCl}_{3}$ molecules. Secondly, the quadruple splitting decreases with increasing temperature, as a result of the decreasing order parameter $\langle S\rangle$. The area under the peaks represent the volume of each phase, and will later be used to determine the temperature dependence of the volume ratio (in chapter 7).

Independent methods have to be used to determine the orientation of the CdSe nanorods. For example, the phosphorous NMR has been performed, because the nanorods are coated with TOPO or/and phosphonic acid molecules. The $\mathrm{P}=\mathrm{O}$ bonds have very large chemical shift anisotropy (> $100 \mathrm{ppm}$ ), i.e. the chemical shift is different for various orientations of $\mathrm{P}=\mathrm{O}$ bond with respect to the magnetic field, and therefore can be used as a convenient internal probe ${ }^{26}$. However, this type of experiment has not been successful 
due to the much lower concentration of the surface-bound molecules comparing with the free ligand molecules in the solution.

Besides the capillary alignment and the magnetic filed induced alignment, shearing the concentrated solution of nanorods between two substrates in a uniform direction has also proven to be successful in aligning the nanorods macroscopically. This is currently a very important method to make thin layers of aligned nanorods for polarization sensitive optical measurements.

In principle, electric field can also be used to align the liquid crystalline solution of the nanorods, due to their anisotropic electric polarizability and their cooperativity in liquid crystalline solutions. This hasn't been succeeded yet, however, because of the practical difficulty in putting the highly viscous liquid crystalline solution between two closely spaced electrodes without solvent loss. Further complications can also be present in the process, such as the migration of charged impurities in the solution and the electrohydrodynamic instability ${ }^{27}$.

In summary, liquid crystalline solution of CdSe nanorods have been made, which at present is tentatively assigned to be the nematic phase. Some success has been achieved in aligning the nanorods by taking advantage of the long-range orientational correlation in the liquid crystalline phase. However, much more has yet to be done, including the final determination of the liquid crystalline phase with SAXS of higher resolution, and the efficient method to have the nanorods aligned on substrates for device applications. 


\section{References}

${ }^{1}$ Chandrasekhar, S. Liquid Crystals, Cambridge Univ. Press: Cambridge 1992.

${ }^{2}$ Robinson, C.; Ward, J. C. Liquid-crystalline structures in polypeptides. Nature 1957, 180 (4596), 1183-1184.

${ }^{3}$ Strzelecka, T. E.; Davidson, M. W.; Rill, R. L. Nature, 1988, 331, 457-460.

${ }^{4}$ Bawden, F. C.; Pirie, N. W.; Bernal, J. D.; Fankuchen, I. Liquid crystalline substances from virus-infected plants. Nature 1936, 138, 1051-1052.

5 Booy, F. P.; Fowler, A. G. Cryo-electron microscopy reveals macromolecular organization within biological liquid-crystals seen in the polarizing microscope. Int. J. Bio. Macromolecules, 1985, 7 (6), 327-335.

${ }^{6}$ Maeda, H.; Maeda, Y. Atomic force microscopy studies for investigating the Smectic structures of colloidal crystals of $\beta$-FeOOH, Langmuir 1996, 12 (6): 1446-1452

${ }^{7}$ Onsager, L. The effects of shape on the interaction of colloidal particles. Ann. N. Y. Acad. Sci. 1949, 51, 627-659.

${ }^{8}$ Vroege, G. J., Lekkerkerker, H. N. W. Phase transitions in lyotropic colloidal and polymer liquid crystals. Rep. Prog. Phys. 1992, 55, 1241-1309.

${ }^{9}$ Straley, J. P. Frank elastic constants of the Hard-rod liquid crystal. Phys. Rev. A 1973, 8, 2181-2183.

${ }^{10}$ Odjik, T. Theory of lyotropic polymer liquid crystals. Macromolecules, 1986, 19 (9), 2313-2319.

${ }^{11}$ Flory, P. J. Molecular Theory of Liquid Crystals. in Advances in polymer science, Vol. 59, Springer-Verlag Berlin 1984, 1-36. 
${ }^{12}$ Frey, M. W., Cuculo, J. A., Ciferri, A., Theil, M. H., A review of lattice theory for lyotropic liquid crystalline polymers, spinodal decomposition, and gel formation. J. M. S. -Rev. Macromol. Chem. Phys., 1995, C35 (2), 287-325.

${ }^{13}$ Flory, P. J. Principles of Polymer Chemistry, Cornell University Press, Ithaca, 1953, 507-509.

${ }^{14}$ Li, L.-S.; Walda, J.; Manna, L.; Alivisatos, A. P. Nano Lett. 2002, 2 (6) 557-560.

${ }^{15}$ Bernal, J. D. and Fankuchen, I. X-ray and Crystallographic Studies of Plant Virus Preparations. J. Gen. Physiology, 1941, 25 (1), 111-165.

${ }^{16}$ Buining, P. A.; Lekkerkerker, H. N. W. Isotropic-Nematic phase separation of a dispersion of organophilic Boehmite rods. J. Phys. Chem. 1993, 97, 11510-11516.

${ }^{17}$ Gunton, J. D., San Miguel, M. and Sahni, P. S. The dynamics of first order phase transition. In Phase Transitions and Critical Phenomena, vol. 8, ed. Domb, C., Lebowitz, J. L. Academic Press, New York, 1983.

${ }^{18}$ Carpineti, M. and Giglio, M. Spinodal-type dynamics in fractal aggregation of colloidal clusters. Phys. Rev. Lett. 1992, 68 (22), 3327-3330.

${ }^{19}$ Cahn, J. W. and Hilliard, J. E. Free energy of a Nonuniform system. J. Chem. Phys. 1958, 28, 258-267.

${ }^{20}$ Li, L.-S., Alivisatos, A. P. Semiconductor nanorod liquid crystals and their assembly on a substrate. Adv. Mat. 2003, 15 (5): 408-412.

${ }^{21}$ Figueredo Neto, A. M. and Amaral, L. Q. Study of Type-I Lyomesophases by X-rayDiffraction. Mol. Cryst. Liq. Cryst. 1981, 74 (1-4), 109-119.

${ }^{22}$ Chandrasekhar, S.; Madhusudana, N. V. Spectroscopy of liquid crystals. Applied Spectroscopy Reviews, 1973, 6, 189-311. 
${ }^{23}$ Seelig, J. Deuterium magnetic resonance: theory and application to lipid membranes. Quart. Rev. Biophys. 1977, 10 (3), 353 - 418.

${ }^{24}$ Forrest, B. J.; Reeves, L. W. New lyotropic liquid crystals composed of finite nonspherical micelles. Chem. Rev. 1981, 81 (1),1-14.

${ }^{25}$ Firouzi, A.; Schaefer, D. J.; Tolbert, S. H.; Stucky, G. D.; Chmelka, B. F. Magneticfield-induced orientational ordering of alkaline lyotropic silicate - surfactant liquid crystals. J. Am. Chem. Soc. 1997, 119 (40), 9466-9477.

${ }^{26}$ Seelig, J. P-31 nuclear magnetic-resonance and head group structure of phospholids in membranes. Biochimi. Biophys. Acta. 1978, 515 (2): 105-140.

${ }^{27}$ De Gennes, P. G. The Physics of Liquid Crystals. Clarendon Press: Oxford, 1974, 187201. 


\section{Chapter 7. Phase Diagram of Solution of CdSe Nanorods}

\subsection{Introduction}

Phase diagrams have played very important roles in determining the interactions in a system. The comparison between theoretically calculated phase diagrams and experimental results reveals the roles of various interactions involved. A famous example is the van der Waals picture of the classical liquids ${ }^{123}$, where the role of the short-range repulsion is predominant in determining the liquid properties. For liquid crystals, various models exist to explain the spontaneous formation of the orientational order. Two extreme cases are Onsager's "rigid rod" model for lyotropic liquid crystals ${ }^{4}$ and the Maier-Saupe model applied for thermotropic liquid crystals ${ }^{5}$, where the anisotropic "soft" interactions are believed to be the most important. Although it has been well accepted nowadays that the short-range repulsive force plays dominant role in the formation of lyotropic liquid crystals, experimental results from a few model systems, such as tobacco mosaic virus (TMV) and fd-virus ${ }^{6}$, have never agreed quantitatively with the rigid rod model, which has been attributed to the presence of the long-range interaction.

With the rod-solvent interaction accounted for by adding the interaction parameter $\chi$, for example, in the Flory's model, the calculated phase diagram has gained temperature dependence, as compared to Onsager's athermal rigid model. In addition, the interrod interactions such as van der Waals interactions and dipole-dipole interaction also introduce temperature dependence due to their anisotropic nature. For example, the 
London dispersion forces between molecules depend on the product of their polarizabilities $\boldsymbol{\alpha}^{7}$. If the polarizabilities are cylindrically symmetric around a long molecular axis, then the London dispersion energy is different for parallel and perpendicular configurations, with its amplitude determined by $\Delta \alpha=\alpha_{\|}-\alpha_{\perp}$. For

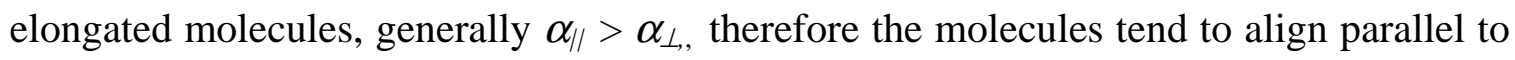
each other. This anisotropic inter-molecular dispersion interaction was first introduced to explain the spontaneous long-range order in small molecular thermotropic nematic liquid crystals $^{5}$.

In order to understand the role of the long-range interaction in the formation of lyotropic liquid crystals, it is necessary to have new model systems with variable aspect ratios. The advent of the methods to make CdSe nanorods with variable but tightly controlled widths and lengths has offered us an opportunity for this kind of study. The CdSe nanorods have large polarizability anisotropy as well as volume dependent permanent electric dipole moment, which, to some extent, may affect the phase diagram. In addition, since the nanorods can be made with tunable size and aspect ratio, the isotropic-nematic phase transition can be shifted to different concentration ranges (due to the rigid rod interaction) so that the contribution from the "soft" interactions can be varied. In this chapter, we study the phase diagram of the isotropic-liquid crystalline phase diagram for the nanorods with a specific width and length within a very narrow temperature range. 


\subsection{Isotropic-Liquid Crystalline Phase Diagram of the Solution of CdSe Nanorods}

In order to determine the temperature vs. composition ( $v s . c$ ) phase diagram of CdSe nanorod solution in a given solvent, the isotropic-biphasic boundary and the biphasic-nematic boundary have to be determined. These boundaries can be seen from the phase diagram calculated with Flory's model (figure 6.4), which have also been observed in some rodlike polymer systems. The techniques we use are optical polarizing microscopy (OPM) with a heating stage and nuclear magnetic resonance (NMR), for the former has high sensitivity to the presence of the ordered phase and the latter to both phases.

Because of the limited quantity of the CdSe nanorods that can be made in each synthesis $(\sim 300 \mathrm{mg})$ and the high viscosity of their concentrated solution, the strategy we adopt here is to make the solution in the biphasic region so that we can first measure the equilibrium concentrations of both the isotropic and the nematic phases $\left(c_{i}\right.$ and $c_{n}$, respectively) at room temperature $\left(25^{\circ} \mathrm{C}\right)$ after the phase separation is completed (e.g. figure 7.1). Then the temperature of the
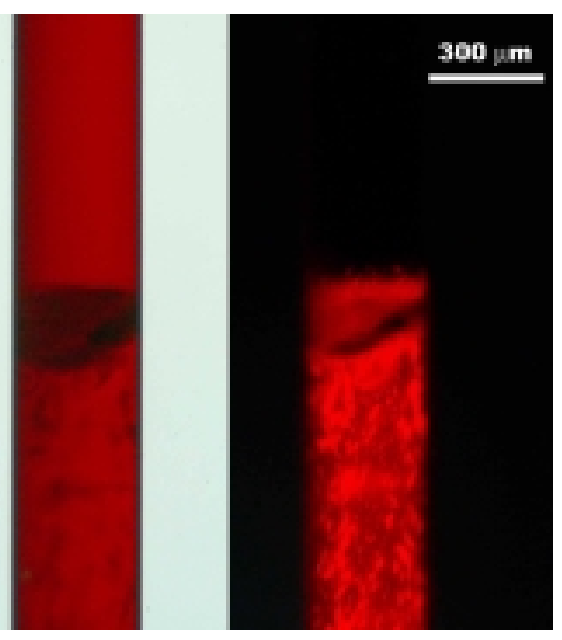

Fig. 7.1 Completion of the isotropic-liquid crystalline phase separation observed between two polarizers, which are parallel for the left figure and crossed for the right. solutions with compositions near $c_{i}$ is varied 
under OPM to observe the disappearance or appearance of the nematic phase so that we can locate the isotropic-biphasic boundary. Once the isotropic-biphasic boundary gets established, the biphasic-nematic boundary can be determined by measuring the isotropic-nematic volume ratio at different temperature with NMR in a solution with known composition. This takes advantage of the non-zero quadruple splitting in the NMR spectra of deuterium nuclei doped in the solution in the ordered phase and zero splitting in the isotropic phase (see chapter 6). This way we can get the temperature dependence of the volume change of both phases.

We start with $3.0 \mathrm{~nm}$ wide and $60 \mathrm{~nm}$ long CdSe nanorods in anhydrous cyclohexane, because their large aspect ratio makes the isotropic-nematic phase transition happen at relatively low concentration so that the phase separation can go to completion in a relatively short period ( 2 months). These nanorods have a width distribution of $\sim 5 \%$, and a length distribution of $\sim 15 \%$.

Nanorod solutions with different compositions are flame-sealed in (nominally) 300 $\mu \mathrm{m}$ diameter cylindrical glass tubes (10 $\mu \mathrm{m}$ wall thickness), and settled for 2 months for completion of phase separation before the concentration measurement was performed. After the phase separation is complete, the glass tubes were immersed in mineral oil (for refractive index matching) and taken digital images under an optical polarizing microscope, so that the actual diameter can be measured along the glass tubes to correct for the possible conic shape. The length of each phase can also be determined, which ranges from tens of microns to a few centimeters depending on the concentration of the solution. Then the glass tubes were cut at the phase boundaries for the concentration determination of each phase. 


\subsubsection{Determination of the Concentration of CdSe Nanorods}

Due to the presence of excess surfactants in dried CdSe powders and the small amount of CdSe nanorods used in the experiment, inductively coupled plasma -atomic emission spectroscopy (ICP-AES or ICP) technique, a common method for elemental analysis nowadays, was used to determine the concentration of the CdSe nanorod solutions. CdSe nanorods were dried and digested in acids, then diluted so that $\left[\mathrm{Cd}^{2+}\right]$ is within the range of 1 to $4000 \mathrm{ppb}$, in which the ICP spectrometer has linear response. The volume ratio of the nanorods in the original solutions can be calculated from the concentration of $\mathrm{Cd}^{2+}$ by assuming all the $\mathrm{Cd}^{2+}$ ions are from CdSe nanorods and taking the density of CdSe as $5.81 \mathrm{~g} / \mathrm{cm}^{3}$ (bulk value). For the $3.0 \times 60 \mathrm{~nm}$ nanorod biphasic solution at room temperature, it was determined that $c_{i}=6.1 \%$ and $c_{a}=9.9 \%$ for pure CdSe, and is the organic shell (5.5 A in thickness) is taken into account, $c_{i}=11.4 \%$ and $c_{a}=18.5 \%$ (volume percentage). These values do not vary for solutions with different compositions, indicating the nanorods are monodisperse enough so that the solution can be considered to be a two-component system, in contrast to the experiments reported for boehmite rods $^{8}$, in which the rods are believed to be bidisperse mixtures.

With the phase boundaries at room temperature known, the concentration of any biphasic solution can be determined as long as the volume ratio of the two phases is known. This provides us a nondestructive way to determine their concentration. 


\subsubsection{Temperature Dependence of the Phase Diagram}

Biphasic boundary is determined by heating biphasic solutions with compositions of near $c_{i}$ under an optical polarizing microscope till the liquid crystalline phase disappears. The composition of the solution is determined by measuring the volume of the isotropic and nematic phases and calculated with $c\left(V_{i}+V_{a}\right)=c_{i} V_{i}+c_{a} V_{a}$. When the solution has a concentration of $6.5 \%$ or $12.1 \%$ (without and with organic shell considered respectively), heating from $25{ }^{\circ} \mathrm{C}$ to $75{ }^{\circ} \mathrm{C}$ does not cause the appearance or disappearance of the nematic phase, therefore we conclude the isotropic-biphasic boundary is very steep, with the variation less than $1 \%$. Considering the thermal expansion of the solvent $\left(\beta=1.14 \times 10^{-3}\right)$ which slightly decreases the concentration, the uncertainty in this boundary should be smaller for higher temperatures.

The determination of the biphasicnematic boundary takes advantage of the quadruple splitting of deuterium nuclei in NMR spectra described in last chapter. Anhydrous deuterated chloroform $\left(\mathrm{CDCl}_{3}\right)$ is added to the biphasic solution of the nanorods in anhydrous cyclohexane $(\sim 5 \%$ by volume) in a $4 \mathrm{~mm}$ diameter glass NMR tube, and the NMR spectra were taken with a $500 \mathrm{MHz}$ spectrometer at

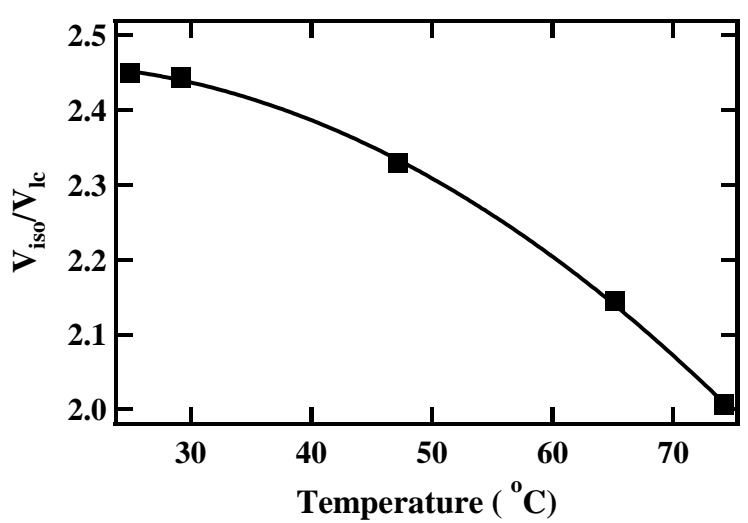

Fig. 7.2 The temperature dependence of the volume ratio between the isotropic and liquid crystalline phases measured by the NMR study. It decreases with increasing temperature. 
temperatures ranging from room temperature to $75^{\circ} \mathrm{C}$. Temperature is varied by blowing compressed air of different temperature over the sample, and calibrated with standard method described below. For each temperature, the sample was allowed to stabilize for 6 $\sim 8$ hours so that the spectra do not change with time. Because $\mathrm{CDCl}_{3}$ probes the local order of the environment, there is no need to wait for the complete phase separation at different temperatures. The D-NMR spectra are shown in figure 6.14, and the central peak was identified to be from the isotropic phase while the two side peaks from the liquid crystalline phase. The volume ratio of the two phases is calculated by the ratio of the corresponding peak areas, and shown in figure 7.2 for different (calibrated) temperatures.

Temperature calibration is achieved using a standard method with ethylene glycol $\left(\left(\mathrm{CH}_{2} \mathrm{OH}\right)_{2}\right)^{9}{ }^{10}$. The difference between the chemical shifts $\Delta \delta$ of the protons in $\mathrm{CH}_{2}$ and $\mathrm{OH}$ groups was measured at different temperatures and the real temperature was calculated with the relationship $\mathrm{T}\left({ }^{\circ} \mathrm{C}\right)=192.7-102.24 \Delta \delta$.

Since the composition of the NMR sample can be calculated from the concentrations and volume ratio of the two phases, the biphasic-liquid crystalline phase boundary is determined with the volume ratio at different temperatures. Here the phase diagram of $3.0 \times 60 \mathrm{~nm}$ CdSe

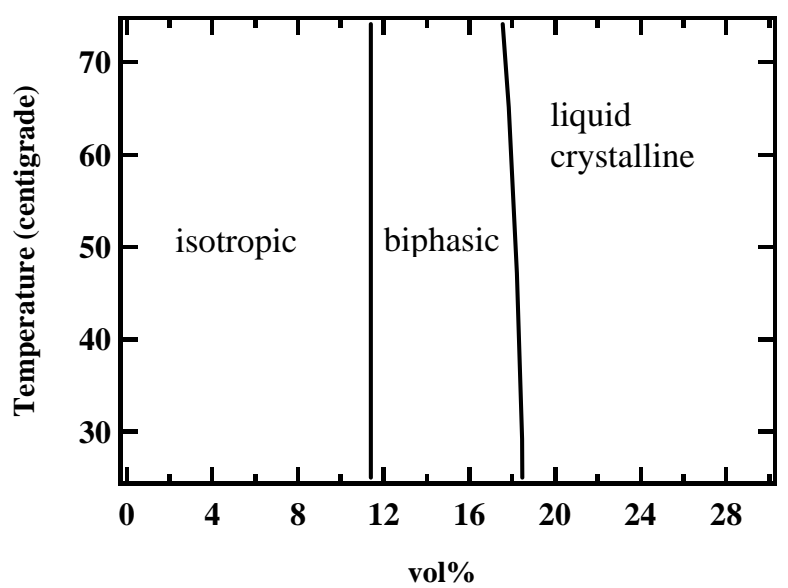

Fig. 7.3 Phase diagram of $3.0 \times 60 \mathrm{~nm} \mathrm{CdSe}$ nanorods in anhydrous cyclohexane. 
nanorods in cyclohexane is determined for the temperature range and shown in figure 7.3.

\subsubsection{Discussion - Comparison with Rigid Rod Model}

Within the temperature range studied (limited by the freezing and boiling of cyclohexane), the temperature dependence of the phase diagram is very weak, qualitatively consistent with the athermal rigid rod model. Comparing with the phase diagram calculated from the lattice model (fig. 6.4), which includes the particle-solvent interaction parameter, the negative slope of the biphasic-liquid crystalline boundary in our measured phase diagram should be caused by the varying rod-solvent interaction parameter. The more enthalpically favored mixing increases the amount of the solvent present in the order phase, accompanied by the decrease in the order parameter with increasing temperature (see Eq. 2, chapter 6).

In order to compare with the theoretical results, the organic capping molecules (1.1nm for double layers) on the nanorods is considered to get an effective diameter of $4.1 \mathrm{~nm}$, and length of $60 \mathrm{~nm}$.

The theoretical results for spherocylinders of aspect ratio 15 in the rigid rod model ${ }^{11}$ 12 give the packing fractions of 0.1916 and 0.2152 for coexisting isotropic and nematic phases respectively, which are significantly higher than our experimental values. In addition, the biphasic region measured experimentally is much wider (1.62 vs. 1.12 for the concentration ratio of nematic phase over isotropic phase). These have also been observed in aqueous suspension of tobacco mosaic virus ${ }^{13}$. On one hand these 
observations may be caused by the polydispersity of the nanorods ${ }^{14}$; on the other hand the attractive interaction between the nanorods may also be responsible.

For two colloidal cylinders with length $L$, radius $R$ and $(d+2 R)$ as the axis-axis distance, the van der Waals ${ }^{15}$ interaction between them can be expressed as $-\frac{A L}{24 d}\left(\frac{R}{d}\right)^{1 / 2}$ and $-\frac{A}{6}\left(\frac{R}{d}\right)$ when they are parallel and perpendicular to each other, respectively, with $\mathrm{A}$ as the Hamaker constant, which is typically $\sim 0.5 \times 10^{-19} \mathrm{~J}(\sim 3600 \mathrm{~K})$. It can be easily shown that for $d \sim 2 R$ (typical for CdSe nanorods in a liquid crystalline solution), the energy difference between the parallel and perpendicular configurations is $\sim(-2) k_{\mathrm{B}} \mathrm{T}$ at room temperature. Numerical study on the rodlike colloids with attractive "deplete" interaction ${ }^{16}$ has shown that introducing orientation-dependent attractive potential results in the broadening of the isotropic-nematic coexisting region, and the decrease in the coexistence concentration of both phases, which is qualitatively consistent with our experimental results.

Another effect caused by the attractive interaction is the narrowing of the nematic region and the stabilization of a smectic phase. This hasn't been observed, because of the high viscosity of the nanorod solution with higher concentrations.

\subsection{Summaries and Future Work}

We have preliminarily measured the isotropic - liquid crystalline phase diagram for CdSe nanorods with a specific size and in a narrow temperature range. In this temperature range the phase diagram shows weak but non-vanishing temperature 
dependence. The coexistence concentrations of the ordered and disordered phases are found to be much smaller then those predicted by the rigid rod model, which may be due to the short-range van der Waals interaction between the nanorods.

In order to systematically study the contribution of short-range interaction to the formation of the ordered phase, comparison between numerical simulations including the van der Waals interaction and experiments on nanorods with different sizes and a wider temperature range is needed. We have found that anhydrous hexanes is also a good solvent for the CdSe nanorods to avoid gelation and to form liquid crystalline phase, and it has much lower freezing point $\left(-95^{\circ} \mathrm{C}\right.$ as compared with $6.5^{\circ} \mathrm{C}$ for cyclohexane). 


\section{Appendix}

\section{A. Concentration Analysis of CdSe solution - ICP Measurement}

Inductively induced plasma - atomic emission spectroscopy (ICP-AES, or simply ICP for short), is a standard multi-element analysis technique that dissociates a sample into its constituent atoms and ions and cause them to emit light at characteristic wavelengths by exciting them to higher energy level. This is accomplished by the use of an inductively coupled plasma source, usually argon. A monochromator separates specific wavelengths of interest, and a detector is used to measures the intensity of the emitted light, which is used to calculate the concentration of corresponding element.

The samples for ICP analysis are generally moderate acidic aqueous solution of the element interested. The standard solutions commercially available are also aqueous and acidic. Thus CdSe

nanorods are digested

with acids for

analysis.

To

determine

the

concentration of $\mathrm{CdSe}$

solution, we chose to

use the concentration

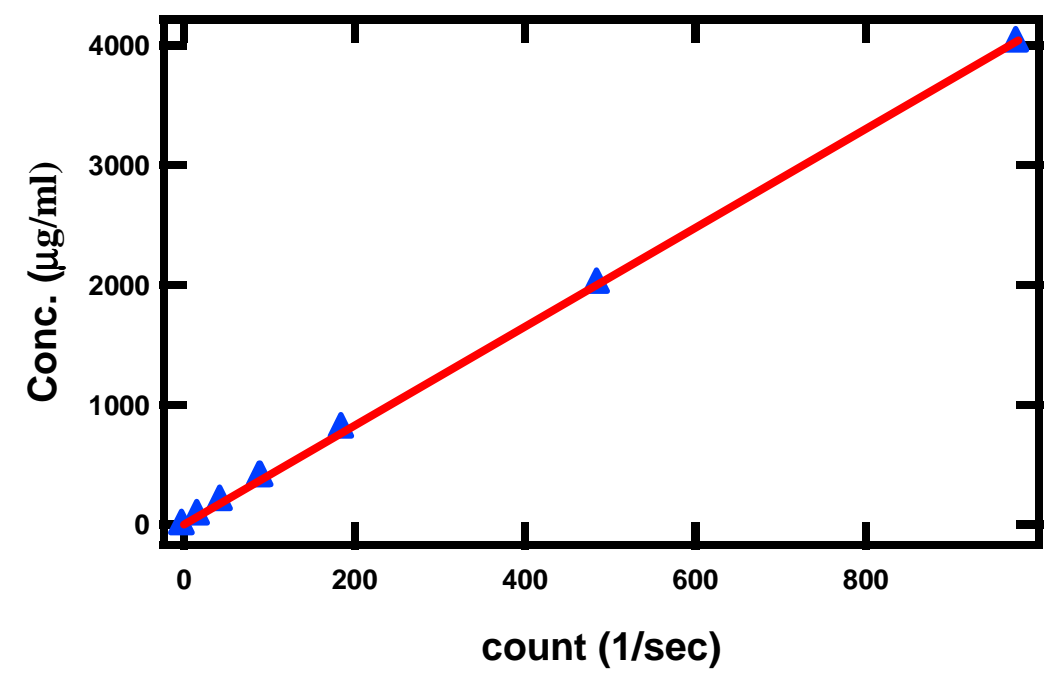

of $\mathrm{Cd}$ element as an

Fig. 7.A1 A typical instrument calibration.

indicator, because the 
loss of Se is inevitable during the digestion. The detection wavelength we use is.214.438 $\mathrm{nm}$, and the siganal is integrated to get the emission intensity.

Calibration of the instrument is done by measuring the response to standard solutions with known Cd concentrations. The standard solution is made by diluting commercial ICP grade Cd standard (Aldrich) with ICP grade nitric acid (Baker, metal content $<1$ ppb) and de-ionized water. It is important to keep the solution acidic all the time to avoid precipitation. Fig 7.A1 shows the calibration of intensity vs. Cd content, showing the instrument response is linear with a wide concentration range for $\mathrm{Cd}$. The calibration has to be done before and after measuring each unknown sample to make sure the response does not drift during the measurement.

CdSe nanorods solution to be determined of a known volume is dried, and the solids are digested in a small amount of ICP grade $\mathrm{HNO}_{3}$ solution (sometimes ICP grade $\mathrm{HCl}$ solution is added to make the digestion faster) and diluted quantitatively to the linear response range of the ICP spectrometer, and analyzed for the Cd content. Thus the concentration of the original solution can be determined. With this method, we also establish the correlation between the UV/vis

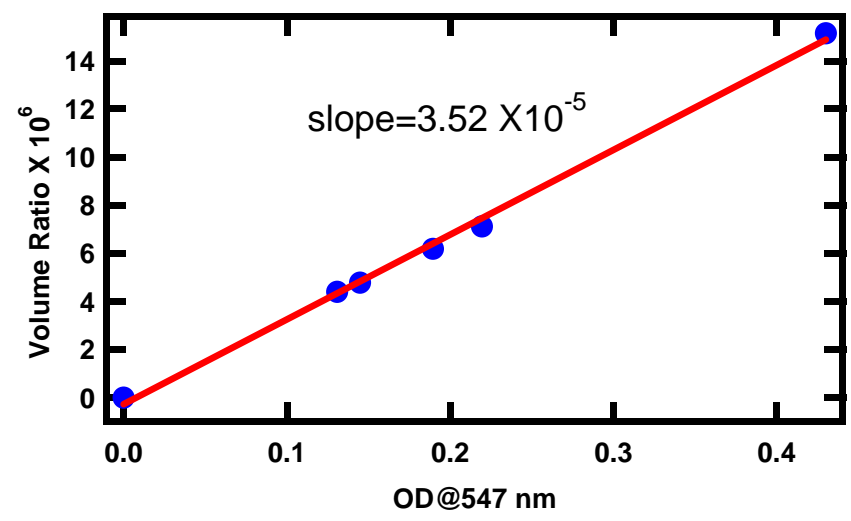

Fig. 7.A2 Volume ratio of $3.0 \times 60 \mathrm{~nm}$ CdSe nanorods in cyclohexane vs. the optical density at $547 \mathrm{~nm}$ (the first excitonic peak). The samples were measured in a $1.0 \mathrm{~cm}$ cell. 
optical density of the nanorods solution and the ICP measured concentration, so that the extinction coefficient of the specific nanorod sample can be determined. As an example, the calibration of the volume percentage of $3.0 \times 60 \mathrm{~nm} \mathrm{CdSe}$ nanorods vs. the optical at their first excitonic peak $(547 \mathrm{~nm})$ is given in figure $7 . \mathrm{A} 2$, from which the extinction coefficient is determined to be $2.84 \times 10^{6} \mathrm{~cm}^{-1}$. 


\section{Reference}

${ }^{1}$ Verlet, L. Computer experiments on classical fluids. Phys. Rev. 1967, 159 (1), 98-103.

${ }^{2}$ Hansen, J.-P. and Verlet, L. Phase transition of the Lennard-Jones system. Phys. Rev. 1969, 184 (1), 151-161.

${ }^{3}$ Chandler, D., Weeks, J. D. and Andersen, H. C. Van der Waals picture of liquids, solids, and phase transformations. Science 1983, 220 (4599), 787-794.

${ }^{4}$ Onsager, L. The effects of shape on the interaction of colloidal particles. Ann. N. Y. Acad. Sci. 1949, 51, 627-659.

${ }^{5}$ Maier, W., Saupe, A. Eine Einfache Molekulare-Statistische Theorie Der Nematischen Kristallinflussigen Phase, Z. Naturforschg, 1959, 14a (10), 882-889; 1960, 15a (4), 288292.

${ }^{6}$ Vroege, G. J.; Lekkerkerker, H. N. W. Phase transitions in lyotropic colloidal and polymer liquid crystals. Rep. Prog. Phys. 1992, 55, 1241-1309.

${ }^{7}$ Israelachvili, J. N. Intermolecular and Surface Forces, Academic Press, London 1985.

${ }^{8}$ Buining, P. A.; Lekkerkerker, H. N. W. Isotropic-nematic phase separation of a dispersion of organophilic boehmite rods. J. Phys. Chem. 1993, 97, 11510-11516.

9 Kaplan, M. L., Bovey, F. A. and Cheng, H. N. Simplified method of calibrating thermometric nuclear magnetic resonance standards. Anal. Chem. 1975, 47 (9), 17031705.

${ }^{10}$ Ammann,C., Meier, P., Merbach,A. E. A simple multi-nuclear NMR thermometer. $J$. Magn. Reson. 1982, 46 (2), 319-321.

${ }^{11}$ Lee, S. -D. A numerical investigation of nematic ordering based on a simple hard-rod model. J. Chem. Phys., 1987, 87 (8), 4972-4974. 
${ }^{12}$ Bolhuis, P. and Frenkel, D. Tracing the phase boundaries of hard spherocylinders. $J$. Chem. Phys. 1998, 106 (2), 666-687.

${ }^{13}$ Fraden, S., Maret, G., Caspar, D. L. D. and Meyer, R. B. Isotropic-nematic phase transition and angular correlations in isotropic suspension of tabacco mosaic virus. Phys. Rev. Lett. 1989, 63 (19), 2068-2071.

${ }^{14}$ Lekkerkerker, H. N. W., Coulon, Ph., Van Der Haegen, R. and Deblieck, R. On the isotropic-liquid crystal phase separation in a solution of rodlike particles of different lengths. J. Chem. Phys. 1984, 80 (7), 3427-3433.

15 Leckband D. and Israelachvili, J. N. Intermolecular forces in biology. Quart. Rev. Biophys. 2001, 34 (2), 105-267.

16 Bolhuis, P. G., Stroobants, A., Frenkel, D. and Lekkerkerker, H. N. W. Numerical study of the phase behavior of rodlike colloids with attractive interactions. J. Chem. Phys. 1997, 107 (5), 1551-1564. 


\section{Chapter 8. Conclusions}

The success in synthesizing monodisperse CdSe nanorods has allowed us to systematically study their physical properties. Compared with the spherical nanoparticles, the elongation of the nanorods causes the appearance of anisotropic properties, such as the linearly polarized photoluminescence, and assembly properties characteristic to geometrically anisotropic objects, such as the formation of the liquid crystalline phases, as described in this thesis.

However, so far the study of anisotropic properties of the nanorods has been limited to the optical properties. The capability to align the nanorods in large scales, when combined with orientation sensitive experimental techniques, would enable us to study other anisotropic properties. For example, we have been trying to use phosphorus NMR to study the selective adsorption of surfactant molecules on different crystal faces, polarizing EXAFS (extended x-ray absorption fine structure spectroscopy) to study the arrangement of the atoms on the surfaces, Terahertz spectroscopy to study the anisotropic carrier conductivity and mobility in the nanorods. Even though we are only in the early stage of these experiments, I believe they will yield important information about the CdSe nanorods.

For the application perspective, the liquid crystalline solution of the nanorods may find their places in devices, such as polarizing light emitting diodes and nanorodconducting polymer composite solar cells. A key step is to develop simple methods to 
make thin layers (e.g. monolayer) of nanorods with controlled orientation on substrates.

In this aspect, we have a lot to learn from the pretreated surface approach that has been widely used in liquid crystal display industry. 